\title{
Scientific poster session
}

\author{
(original and unedited texts as received by the authors)
}

\author{
Advanced Imaging Modalities \\ P1:1

\section{SPONTANEOUS THIRD-VENTRICULOSTOMY: EN VALVE MECHANISM REVEALED BY FLOW-SENSITIVE PHASE-CONTRAST CINE MRI}

G. ZUCCOLI $^{1}$, N. PIPITONE ${ }^{2}$

${ }^{1}$ University of Pittsburgh, PA, USA, ${ }^{2}$ Arcispedale Santa Maria Nuova, Reggio Emilia, ITALY

\section{PURPOSE}

Spontaneous third ventriculostomy (STV) is a rare condition in which the cerebrospinal fluid (CSF) bypasses the cerebral aqueduct flowing from the inferior third ventricle into the interpeduncular cistern. Flow-sensitive phase-contrast (PC) cine Magnetic Resonance Imaging (MRI) can demonstrate the flow from the third ventricle floor. The aim of this study was to describe the on-off movement showed by PC cine MRI stationary tissue analysis.

METHODS

Flow-sensitive PC MRI was performed emphasizing both the relative CSF pulsation and stationary tissue characteristics. Clinical data of the patient was obtained.

\section{RESULTS}

A 56-year-old epileptic woman affected by hydrocephalus due to aqueductal stenosis underwent brain MRI. The size of the ventricular system was unchanged from previous examinations. Flow-sensitive phase-contrast cine MRI showed flow absence through the aqueduct and flow pulsations through the third ventricle floor which showed an on-off movement on stationary tissue images.

CONCLUSION

Spontaneous ventriculostomy is a rare condition occurring in chronic obstructive hydrocephalus patients as a result of long-term pulsations against third ventricle floor. On-off movements of the third ventricle floor could account for chronic hydrocephalus persistence by an "en valve" mechanism.

\section{P1:2}

\section{FOUR-DIMENSIONAL MR IMAGING OF NORMAL AND PATHOLOGICAL PITUITARY GLAND BY USING TRICKS-XV}

A. WADA, S. Hara, S. Ozaki, T. Yoshizako, A. Matsumura, T. Hayashi, Y. Tsuchie, N. Yamamoto, H. Kitagaki Shimane University, Department of Radiology, Izumo, JAPAN
PURPOSE

TRICKS (time-resolved imaging of contrast kinetics) is a recent three-dimensional (3D) gradient-echo sequence with characteristic $3 \mathrm{D} \mathrm{k}$-space segmentation and data recombination. Additional parallel imaging technique (TRICKS-XV) contributes to higher spatial resolution (sub-square millimeter) 3D imaging without sacrificing spatial resolution. In this study, we tried to adapt TRICKS-XV to dynamic MR imaging of the pituitary gland and evaluate the utility of time-resolved 3D (=4D) imaging in deal with pituitary adenoma. MATERIALS AND METHODS

Time-resolved 3D dynamic MR imaging was performed in three normal (no pituitary gland lesion) and five pathological subjects. All MR examinations were performed on 3.0T MR unit (Signa HDx 3.0: GE Healthcare) with 8-channel head coil. Parameters of TRICKS-XV were following; TR/TE: $4.48 / 1.36 \mathrm{msec}$, FA: 15 , FOV: $20 \times 20 \mathrm{~cm}$, matrix: $256 \times$ 256, slice thickness: $0.8 \mathrm{~mm}$, gapless 32 slices, coronal plane, acquisition time: $5.3 \mathrm{sec} /$ phase. Continuous 15 phases $3 \mathrm{D}$ isotropic imaging $(0.8 \times$ $0.8 \times 0.8 \mathrm{~mm}$ ) data accumulation after $10 \mathrm{ml}$ Gd-DTPA and following $20 \mathrm{ml}$ saline injection with fixed flow rate $(2 \mathrm{ml} / \mathrm{sec})$ was performed. RESULTS

In all three normal subjects, progress of the contrast enhancement effect from pituitary stalk to anterior lobe and early contrast enhancement of posterior lobe were recognized. Color-scaled 3D viewing revealed the extension of contrast enhancement effect distinctly. Multiplanar projection technique based on 3D isotropic imaging was useful to evaluate the location and extension of microadenoma. High temporal resolution of TRICKS-XV depicted a change of an intratumoral enhancement effect. CONCLUSION

High resolution 4D MR imaging using TRICKS-XV is useful for the evaluation of normal and pathological pituitary glands.

\section{P1:3}

\section{AN ATTEMPT TO PREPARE HIGH-RESOLUTION IMAGES WITH WHICH CRANIAL NERVES CAN BE EASILY DIFFERENTIATED FROM CRANIAL VESSELS}

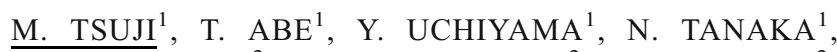
R. KATAYAMA ${ }^{2}$, M. YAMAGUCHI ${ }^{2}$, N. KIKUCHI ${ }^{2}$, N. HAYABUCHI ${ }^{1}$

${ }^{1}$ KURUME UNIVERSITY SCHOOL OF MEDICINE, KURUME, JAPAN, ${ }^{2}$ CENTER FOR DIAGNOSTIC IMAGING KURUME UNVERSITY HOSPITAL, KURUME, JAPAN

\section{PURPOSE}

To ascertaining structures around the brainstem, reverse images of heavy T2-weighted images are widely used. However when 
differentiating cranial nerves from cranial vessels, anatomical knowledge is currently more important than signal intensity of them. The aim of this study is to reconstruct fusion images with which cranial nerves could be differentiated from cerebral arteries in posterior fossa based on signal intensity.

\section{METHODS}

Magnetic resonance cisternography and TOF-MRA were performed with a clinical MR imager (Signa HDx 3.0 Tesla). These image data sets were obtained in the same volume area. The MR images were transferred to workstation (Advantage windows Ver4.3). DICOM data were uploaded to a personal computer with Windows XP and were converted into 8-bit files, and Image J was used for image synthesis.

\section{RESULTS}

Image-J software was used for image processing, successfully. Fusion image in the volumetric region was obtained in the difference gray scale of cranial nerves and vessels. Anatomical relationship of the cranial nerves and vessels was well shown in the multi planer reconstruction image.

\section{CONCLUSIONS}

The 3-demensional STIR with reverse image can demonstrate similar contrast, but the limited MR system can acquire the images. However, the present useful method is inexpensive and convenient for every MR system. While arteries could be differentiated from nerves, delineation of veins will be necessary. One problem was that there were individual and age differences in MRA signal intensity and contrast. This method could be one of possibilities for 3D image formation of the posterior fossa.

\section{P1:4}

\section{CROSSED CEREBELLAR DIASCHISIS DUE TO INTRACRANIAL HEMATOMA IN BASAL GANGLIA OR THALAMUS: ASSESSMENT WITH DIFFUSION TENSOR MR IMAGING}

\author{
H. SONG ${ }^{1}$, Y. Kim ${ }^{2}$ \\ ${ }^{1}$ Cheju Halla General Hospital, Jeju, SOUTH KOREA, ${ }^{2}$ Uijongbu \\ St. Mary's Hospital, Uijongbu, SOUTH KOREA
}

\section{PURPOSE}

Diaschisis refers to a functional impairment occurring remotely from the site of a brain lesion, which is anatomically connected by fiber tracts. The purpose of our study was to evaluate whether diffusion tensor MR imaging (DTI) might provide evidence of crossed cerebellar diaschisis (CCD) in patients with unilateral deep-seated hematoma without cortical structural abnormality.

METHODS

Fourteen patients ( 9 men, 5 wome; mean age 56.4 years) with unilateral hypertensive intracerebral hemorrhage strictly confined either to the basal ganglia or thalamus were evaluated by DTI. Nine psychiatric patients without structural abnormality on MRI were included as control subjects. For the evaluation of pontocerebellar fibers, the fractional anisotrophy (FA) of the bilateral middle cerebellar peduncle (MCP) in both patient and control groups was measured. Changes of FA values in the MCP were compared.

RESULTS

In patients with a intracranial hematoma in basal ganglia or thalamus, MCP of the contralesional side showed an FA value of $0.6812+-0.0174$, which was significantly lower than that of the ipsilateral side MCP $(0.7201+-0.0174)$ (one tail paired t test, $\mathrm{p}=.0009)$.

CONCLUSIONS

DTI can visualize CCD in patients with subcortical hematoma without cortical structural abnormality. This suggested that CCD can develop regardless of interruption of the corticocerebellar tract, which is the pricipal pathway of $\mathrm{CCD}$,

\section{P1:5}

\section{COMPUTER AIDED DETECTION AND DIAGNOSIS IN STROKE}

\section{K. SKLINDA $^{1}$, J. Walecki ${ }^{2}$, A. Przelaskowski ${ }^{3}$}

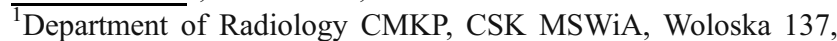
Warsaw, POLAND, ${ }^{2}$ Department of Experimental Pharmacology Polish Academy of Sciences Medical Research Center, Pawinskiego 5, Warsaw, POLAND, ${ }^{3}$ Institute of Radioelectronics, Warsaw University of Technology Nowowiejska 15/19, Warsaw, POLAND

\section{PURPOSE}

Stroke is a syndrome characterized by a sudden neurological deficit caused by cerebra ischemia. Computed tomography (CT) plays a crucial role in the evaluation of stroke patients even though it is not sufficient enough in the meaning of extraction of hypodense area corresponding with infracted cerebral tissues in hyperacute stage. Careful selection of patients for a thrombolytic therapy is fundamental to improve safety and efficacy, therefore we propose new waveletbased computer-aided detection and diagnosis (CADD) method of extraction of hypodensity in CT scans corresponding with cytotoxic/ early vasogenic edema in stroke.

\section{METHODS}

Evaluation of 123 sets of examinations conducted in patients admitted with symptoms suggestive of stroke was undertaken by experienced neuroradiologists and by residents. All of the cases selected for further evaluation and follow up study were considered as having no direct signs of hyperacute ischemia in the localization corresponding with clinical manifestation. Three of the observers performed the evaluation retrospectively, four of them - prospectively. All sets were evaluated independently. Follow-up CT exam and/or clinical picture confirmed or excluded the diagnosis of stroke.

RESULTS

Higher AUC values were found for CADD for all radiologists and differences were significant for all subgroups $(\mathrm{p}<0,05)$ of CT examinations, taking into account the quality of scans, artifacts, asymmetry, scarification, and severe cerebral atrophy which may influence the radiological diagnosis. Mean sensitivity value for all observers increased from $38,5 \%$ (CT) to $51,3 \%$ (CADD). 


\section{CONCLUSIONS}

Combining the results of CT and CADD provided a better diagnosis in all cases of stroke, which is potentially beneficial for stroke patients and proves usefulness of CADD in brain CT examinations. Planned prospective studies will let evaluate the impact on further treatment of hyperacute stroke patients and correlation with perfusion- $-\mathrm{CT}$ and perfusion-MR imaging.

\section{P1:6}

\section{CORTICAL REPRESENTATION OF DIFFERENT TASTANTS IN THE HUMAN PRIMARY GUSTATORY REGION: AN FMRI STUDY}

G. POLONARA ${ }^{1}$, F. FIORI ${ }^{2}$, G. MASCIOLI ${ }^{1}$, M. FABRI ${ }^{2}$, T. MANZONI ${ }^{2}$, U. SALVOLINI ${ }^{1}$

${ }^{1}$ Sezione Scienze Radiologiche, Dipartimento di Scienze Cliniche Specialistiche,Università Politecnica delle Marche, ANCONA, ITALY, ${ }^{2}$ Sezione di Fisiologia Dipartimento di Neuroscienze, Università Politecnica delle Marche, ANCONA, ITALY

\section{PURPOSE}

To establish the topographical organization of the BOLD effect elicited by four taste stimuli (salty, sweet, sour, and bitter) and by a neutral stimulus (distilled water) in the human primary gustatory area (GI) of the fronto-parietal operculum.

\section{MATERIALS \& METHODS}

Unilateral stimulation of the tongue (2-5 taste stimuli) was applied to 15 healthy subjects ( 10 women, 5 men) using 5-min fMRI block-design protocols. The stimulation paradigm envisaged alternate periods of stimulation and rest. The protocol was approved by the local Ethics Committee. Data were analyzed with BrainVoyager, SPM2 and Functool softwares.

\section{RESULTS}

In all subjects unilateral taste stimulation with the different tastants, including water, evoked bilateral activation in area GI. Foci were generally wider and signal increases greater in ipsilateral area GI.

The mean Talairach coordinates of the foci evoked by the different tastants were slightly but not significantly different $(\mathrm{p}>0.05)$, with a wide degree of overlap between the cortical areas activated by each taste stimulus. In addition, activations exhibited considerable inter-individual variability.

\section{CONCLUSION}

The present data confirm previous neuropsychological and functional findings suggesting that the gustatory pathways from tongue to cortex are distributed bilaterally, with an ipsilateral predominance. However, a clear topographical organization of the cortical areas activated by specific taste stimuli (chemiotopy) could not be recognized.

A possible explanation for these findings is that the fMRI technique is unable to resolve fine topographical arrangements. On the other hand, the discriminative role of area GI for the different tastants could be subserved by a non-spatial mechanism. Both explanations may also apply.

Finally, the considerable inter-individual variability of activation data, likely depending on individual cerebral functional anatomy, is in line with the notion that tastes are perceived differently by different subjects.

\section{P1:7}

\section{TOPOGRAPHY OF CALLOSAL FIBRES CONNECTING THE PRIMARY VISUAL AREAS: A DTI AND FMRI STUDY IN CONTROL SUBJECTS AND CALLOSOTOMIZED PATIENTS}

\author{
G. POLONARA ${ }^{1}$, G. MASCIOLI ${ }^{1}$, M. FABRI ${ }^{2}$, G.L. CAVOLA ${ }^{1}$, \\ $\overline{\text { S. SALVOLINI }}^{2}$, T. MANZONI ${ }^{2}$, U. SALVOLINI ${ }^{1}$ \\ ${ }^{1}$ Università Politecnica delle Marche, Sezione Scienze \\ Radiologiche, Dipartimento di Scienze Cliniche Specialistiche, \\ Ancona, ITALY, ${ }^{2}$ Università Politecnica delle Marche, Diparti- \\ mento di Neuroscienze, Ancona, ITALY
}

\section{PURPOSE}

Previous studies of human callosal lesions and anatomical tracing investigations in other mammals have described a topographical organization of the interhemispheric fibres connecting homologous cortical regions. Human anatomical studies suggested that the fibres connecting the visual cortices in the occipital lobes cross the corpus callosum (CC) through its posterior part. This study was designed to confirm the involvement of the splenium in the transfer and integration of visual information, by correlating anatomical and functional data collected with DTI and fMRI.

\section{METHODS}

Twelve healthy volunteers and 9 callosotomized subjects participated in the study. Callosal resections, performed to treat severe epilepsy, ranged from total callosotomy $(n=5)$ to partial anterior resection $(n=3)$ and posterior callosotomy $(n=1)$. Data were collected using a $1.5 \mathrm{~T}$ magnet. Visual stimulation was applied to 7 control subjects and to 2 patients, one with anterior and the other with total callosotomy. fMRI stimuli were a black and white checkerboard presented to the central or peripheral visual field according to a block-design protocol that envisaged 15 -sec periods of rest and stimulation. The anatomical correlates of functional activation were investigated with DTI and diffusion tensor tractography (DTT) in subjects and patients.

\section{RESULTS}

The DTT data showed that fibre bundles arising in cortical regions of interest selected in the left and right primary visual cortex crossed through the splenium both in control subjects and in patients with anterior callosotomy. In the patients with posterior and complete resection, fibres from the occipital lobes did not reach the contralateral hemisphere. An activation focus in the splenium was seen in all subjects and in the patient with anterior callosotomy who received visual stimulation in the same region where the interhemispheric fibres crossed through the CC.

\section{CONCLUSION}

Combined anatomical and functional investigations confirmed that the splenium is involved in the interhemispheric transfer of information between visual cortical areas. 
P1:8

\section{THE COMPARISON OF PROPELLER FLAIR AND STANDARD FLAIR}

M. MAEDA, N. Matsushima, K. Takeda

Mie University School of Medicine, Department of Radiology, Tsu, JAPAN

\section{PURPOSE}

Periodically Rotated Overlapping ParallEL Lines with Enhanced Reconstruction (PROPELLER; BLADE) techniques have been proposed to reduce the effects of head motion. The aim of our exhibition is to demonstrate clinical usefulness and limitations of PROPELLER FLAIR in comparison with standard FLAIR.

METHODS

Consecutive 73 patients who gave consent were included in a prospective comparison of PROPELLER and standard FLAIR sequences. CNS diseases included infarction, multiple sclerosis, tumors, and miscellaneous lesions. All examinations were performed at $1.5 \mathrm{~T}$ with comparison of standard T2-weighted FLAIR to PROPELLER T2-weighted FLAIR in the axial image orientation. Imaging protocols were matched for spatial resolution, with data evaluation by 2 experienced neuroradiologists. Image data were compared qualitatively and quantitatively regarding various image artifacts (motion, CSF flow, pulsation, and metal artifacts) and overall image quality (conspicuity and detection).

RESULTS

Qualitatively, PROPELLER FLAIR showed significantly less motion, CSF flow, and pulsation artifacts (particularly from transverse sinuses) than the standard FLAIR. However, conspicuity and detection of lesions were better in standard FLAIR than in PROPELLER FLAIR. Quantitatively, Lesion contrast (lesion signal - adjacent normal parenchymal signal/adjacent normal parenchymal signal) was significantly lower in PROPELLER than the standard FLAIR.

CONCLUSION

PROPELLER FLAIR had disadvantage in lesion conspicuity compared with the standard FLAIR. However, PROPELLER FLAIR significantly reduces various artifacts including motion, pulsation, metal and CSF flow artifacts. Those results appear advantageous particularly in children or emergent patients.

\section{P1:9}

\section{PREOPERATIVE IDENTIFICATION OF THE CENTRAL SULCUS USING FUNCTIONAL MRI WITH EPI SEQUENCES}

A. LÜTTICH, A.M. DIEGUEZ, J. ZAMARRO PARRA, M. ESPINOSA de RUEDA, G. PARRILLA

Hospital Universitario Virgen de la Arrixaca, Murcia, SPAIN

\section{BACKGROUND AND PURPOSE}

Functional magnetic resonance imaging (fMRI) is a noninvasive neuroimaging technique that enables the visualization of vascular changes originating in the cortex on the execution of a simple motor task. We aim to delimit the usefulness of motor fMRI using echo-planar imaging (EPI) techniques and assess its clinical usefulness in the identification of the central sulcus.

\section{METHODS}

We studied 32 candidates for neurosurgery who had centrally located space-occupying lesions with fMRI using EPI images with blood oxygen level-dependent (BOLD) gradient-echo (GE) sequences acquired on a $1.5 \mathrm{~T}$ scanner while patients repeatedly opened and closed their hands. Statistical activation images ( $t$ images) corresponding to the movements of the right and left hands were compared using cancellation analysis. Three-dimensional reconstruction of the cranium and brain of each patient showed the relative position of the expansive lesion and of non-damaged cortical tissue. RESULTS

Reproducible and selective functional motor activation was observed in all patients; this activation enabled the central sulcus to be identified in all cases.

CONCLUSION

fMRI using GE EPI sequences in combination with threedimensional reconstruction is a useful and easy technique to functionally identify the motor cortex.

\section{P1:10}

\section{QUANTITATIVE DTI TRACTOGRAPHY OF PATIENTS WITH SYMPTOMS AFTER MILD TRAUMATIC BRAIN INJURY}

\section{T. KURKI}

Pulssi Medical Center, Turku, FINLAND

\section{PURPOSE}

Conventional MRI can generally not detect changes in patients with symptoms after mild traumatic brain injury. Diffusion tensor imaging (DTI) is able to demonstrate changes not visible in conventional images. In this study, DTI with fiber tracking was performed in patients with and without cognitive sequalae after mild traumatic brain injury.

\section{MATERIALS AND METHODS}

Clinical patients studied for symptoms after mild traumatic brain injury and without traumatic findings in MRI (intracranial haemorrhage, other traumatic lesions or posttraumatic atrophy) were enrolled into the study. Of these 26 patients 15 had cognitive (group A) and 11 other posttraumatic symptoms (group B). MRI was performed at $3 \mathrm{~T}$, the voxel size in DTI was $2 \times 2 \times 2 \mathrm{~mm}$. Based on DTI tractography volume, mean FA and mean ADC were measured in corpus callosum (CC), cingulum, superior longitudinal fasciculus, uncinate fasciculus (UF), inferior fronto-occipital fasciculus and projection fibers through anterior corona radiata (ACR). The results were compared to those of 14 normal persons.

\section{RESULTS}

Of the 286 measurements, a volume $<2 \mathrm{SD} / 3 \mathrm{SD}$ was detected in $10 / 0$ times, a FA value $<2 \mathrm{SD} / 3 \mathrm{SD}$ was detected $13 / 4$ times and an ADC value $>2 \mathrm{SD} / 3 \mathrm{SD} 16 / 7$ times. Two CCs, one cingulum, one right ACR, three left ACRs, three right UFs and one left UF were defined as pathological (value $>3$ SD of any parameter or value $>2$ SD of two parameters). Statistically significant difference was noted in the volume of the left ACR between group A and normal persons.

\section{CONCLUSION}

Quantitative DTI tractography at $3 \mathrm{~T}$ can reveal white matter chafter mild traumatic injury. Of the tracts studied, anterior corona radiata and uncinate fasciculus seem to be most often damaged structures. 
P1:11

\section{DIFFERENTIATION BY MR SPECTROSCOPY BETWEEN RADIONECROSIS AND TUMOR RELAPSE OF SURGICAL REMOVED BRAIN GLIOMAS}

S. LYRA $^{1}$, V.K. KATSAROS ${ }^{2}$, E. SAMARAS ${ }^{3}$, A. PAPATHEODOROU ${ }^{1}$, N. DELIKANAKIS ${ }^{1}$, D. GAVRILOU ${ }^{1}$, V. VARSOS ${ }^{3}$, N. BATAKIS ${ }^{1}$

${ }^{1}$ RED CROSS HOSPITAL, DEPARTMENT OF RADIOLOGY, ATHENS, GREECE, ${ }^{2}$ IKA ONCOLOGY HOSPITAL, DEPARTMENT OF CT AND MRI, ATHENS, GREECE, ${ }^{3}$ RED CROSS HOSPITAL, CLINIC OF NEUROSURGERY, ATHENS, GREECE

\section{PURPOSE}

To prospectively investigate the ability of MR spectroscopy in the differentiation of brain tumor relapse and post-radiation necrosis. MATERIALS AND METHODS

14 examinations (13 patients) with a history of brain tumor which was treated by a combination of surgery, radiotherapy and chemotherapy were studied. The initial pathologic diagnosis showed Grade II-IV glioma in all patients. The follow-up control by MR spectroscopy was performed between the 4th and 18th month after the surgery, except for one in whom the control was performed 40 months after the surgery. The Cho/NAA ratio was evaluated. All patients had also a follow-up pathology.

RESULTS

In 8 patients the Cho/NAA ratio was bigger than $2(2.1-17)$ and the follow-up pathologic examination confirmed tumor relapse. In two cases in which the Cho/NAA ratio was smaller than 2 (1.7 and 1.8), the follow-up pathologic examination showed tumor relapse, while in the remaining three patients where the Cho/NAA ratio was smaller than $2(1.2,1.3$ and 1.4 respectively) the follow-up pathologic examination showed radionecrosis.

CONCLUSION

Although our series number is not so big, the results are indicative that the Cho/NAA ratio $>2$ represents tumor relapse, while Cho/ NAA ratio $<2$ does not constitute differentiation element in the discrimination of radionecrosis and tumor relapse.

\section{P1:12}

\section{CONFOUNDING EFFECTS OF MAGNETIC FIELDS AND GRADIENT NOISE OF MRI SCANNER ON MULTIMODAL MR-ELECTROENCEPHALOGRAPHY}

\author{
S. KARAKAS ${ }^{1}$, E. DOGUTEPE-DINCER ${ }^{1}$, A. OZKAN-CEYLAN ${ }^{1}$, \\ Z. BARAN ${ }^{1}$, H.M. KARAKAS ${ }^{2}$, E.T. TALI ${ }^{2}$ \\ ${ }^{1}$ HACETTEPE UNIVERSITY - COGNITIVE PSYCHOPHYSIOL- \\ OGY RESEARCH CENTER, ANKARA, TURKEY, ${ }^{2}$ GAZI UNI- \\ VERSITY - DEPARTMENT OF RADIOLOGY - SECTION OF \\ NEURORADIOLOGY, ANKARA, TURKEY
}

\section{PURPOSE}

Selective effects of electromagnetic fields and acoustic noise (AN) on auditory event-related potentials (ERPs) during multimodal electroencephalography (MRI-EEG/ERP) were investigated for early sensory (N100) and late cognitive (P300) components.

\section{METHODS}

23 healthy volunteers (age: $21.61 \pm 2.04$ ) were studied. ERPs were recorded under the passive auditory oddball paradigm. Stimuli $(75 \mathrm{msec})$ were delivered in a series of rare tones that were randomly distributed amongst frequent tones. AN was recorded and simulated in electrophysiology laboratory (Lab-e). 64-channel EEG was performed in MRI environment (MRI-e) during fMRI scan (both electromagnetic interference and $\mathrm{AN}$ ); after the scan (only the main magnetic field); and in Lab-e, with and without the presence of simulated AN. The effect of environment (MRI-e and Lab-e), AN (real, simulated) and recording site (Fz, Pz) was tested using a $2 \times 2 \times 2$ ANOVA-RM. Separate ANOVAs were performed on component amplitudes and latencies.

RESULTS

ERP waveforms, recorded under four recording conditions, were morphologically similar. MRI-e had a significant effect on the latency of $\mathrm{N} 100,[\mathrm{~F}(1,21)=8.270, \mathrm{p}=.009$, Partial eta $2=.283]$ and the amplitude of $\mathrm{P} 300[\mathrm{~F}(1,21)=5.386, \mathrm{p}=.03$, Partial eta2 $=.204]$. AN had a significant effect on the latency of both $\mathrm{N} 100[\mathrm{~F}(1,21)=33.056, \mathrm{p}=.0001$, Partial eta $2=.612]$ and of $\mathrm{P} 300$ $[\mathrm{F}(1,21)=21.865, \mathrm{p}=.0001$, Partial eta2 $=.510]$. The 2-way and 3 way interaction effects that involved recording environment and AN were not significant, showing that the effect of AN was constant for two recording environments.

CONCLUSIONS

Electromagnetic interference and acoustic noise delay sensory processing (N100 latency). Amplitude of the late cognitive potential, P300, was higher during MRI and appeared later during acoustic noise. These variations indicate effortful processing and increased cognitive load in MRI. Morphological similarity of the waveforms in four environments shows that MRI-EEG/ERP is possible, but practical applications in neuroradiology require expert knowledge and focused training on this newly emerging multimodal technology.

\section{P1:13}

\section{CEREBRAL MAGNETIC RESONANCE SPECTROSCOPY AT 7 TESLA: STANDARD VALUES AND REGIONAL DIFFERENCES}

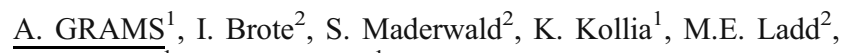
$\overline{\text { M. Forsting }}^{1}$, E.R. Gizewski ${ }^{1}$

${ }^{1}$ Department of Diagnostic and Interventional Radiology and Neuroradiology, Essen, GERMANY, ${ }^{2}$ Erwin L. Hahn Institute for Magnetic Resonance Imaging, Essen, GERMANY

\section{PURPOSE}

Regional differences of cerebral metabolite distributions have been described, whereas this data is extremely controversial and mainly based on examinations with 1.5 Tesla scanners. 7 Tesla magnetic resonance spectroscopy (MRS) is expected to deliver a higher spectral resolution and a better distinguishability between different metabolites than scanners with lower field strengths (1.5 / 3 Tesla). On the other hand problems which minimize spectral quality may arise in a higher magnetic field. As there are only a few studies in 
humans so far, no standard values for 7 Tesla concerning regional metabolite distributions and concentrations are existent.

\section{METHODS}

In the present study some metabolites detectable with cerebral (1H) MRS: N-acetyl-aspartate (NAA), choline (Cho) and creatine $(\mathrm{Cr})$, were evaluated in 10 healthy volunteers with a $(1-\mathrm{H})$ single voxel sequence (PRESS, TE: $30 \mathrm{~ms}$ ). 5 voxels were placed in frontal, parietal and cerebellar white matter, as well as in insular occipital and thalamic grey matter respectively.

\section{RESULTS}

For NAA the lowest values were found in frontal white, the highest in thalamic grey matter. Cho displayed the lowest values in frontal white und the highest in insular grey matter. Cr showed the lowest values in frontal white and the highest in thalamic grey matter.

The uppermost Cho/Cr ratio could be described in parietal white, the least in thalamic grey matter. The highest NAA/Cr ratio was found to be in thalamic grey, the lowest in frontal white matter. CONCLUSIONS

Thus, in the present study we were able to verify regional metabolite differences with in vivo high field MRS. Quantitative values and metabolite ratios could be a basis for further clinical studies.

\section{P1:14}

\section{MAGNETIC RESONANCE IMAGING IN TRAUMATIC BRAIN INJURY: A COMPARISON BETWEEN MR SEQUENCES IN INTER-RATER RELIABILITY, LESION DETECTION AND RELATIONSHIP WITH OUTCOME}

\author{
B.H.J. Geurts ${ }^{1,2}$, T.M.J.C. Andriessen ${ }^{2}$, P.E. $\operatorname{Vos}^{2}$, B.M. Goraj ${ }^{1}$ \\ ${ }^{1}$ UMCN St Radboud Radiology, Nijmegen, THE NETHERLANDS, \\ ${ }^{2}$ UMCN St Radboud Neurology, Nijmegen, THE NETHERLANDS
}

\section{PURPOSE}

To compare T2-weighted imaging (T2WI), Fluid Attenuated Inversion Recovery (FLAIR), T2*-gradient recalled echo (T2*GRE) and Susceptibility Weighted Imaging (SWI) in inter-rater reliability, lesion detection and outcome prediction in patients with traumatic brain injury (TBI).

METHODS

MRI images of 58 TBI patients were retrospectively assessed. Each sequence was scored independently from the others on number of traumatic punctate lesions and lesion volume. Twenty images were assessed by four raters, one rater scored the remaining images. Injury severity was assessed with the Glasgow Coma Scale (GCS) at the emergency department. Functional outcome was assessed with the Glasgow Outcome Scale Extended (GOSE) at time of MRI.

\section{RESULTS}

Inter-rater reliability was good for lesion volume in all sequences and punctate lesions on T2*-GRE and SWI (ICC between .84 and .93 ) but was poor for punctate lesions on T2WI and FLAIR (ICC ¡Ü .13). T2*-GRE revealed a significantly lower lesion volume compared to the other sequences. SWI showed significantly more punctate lesions compared to T2*-GRE, which in turn revealed more lesions than FLAIR and T2WI. Number of punctate lesions on T2*-GRE $(\mathrm{r}=.51, \mathrm{p}<.001)$ and SWI $(\mathrm{r}=.56, \mathrm{p}<.001)$ correlated with the GCS while SWI lesion volume correlated with the GOSE $(\mathrm{r}=.41, \mathrm{p}=.002)$.

CONCLUSION

Visually detected traumatic punctate lesions on T2WI and FLAIR should be interpreted with caution since there is considerable disagreement between raters. Since SWI was equal or superior to the other sequences in the detection of lesions and showed best correlations with injury severity and outcome, it should be included in MRI protocols in TBI patients.

\section{P1:15}

\section{REPRODUCIBILITY OF A FAST RETINOTOPIC MAPPING FMRI PROTOCOL}

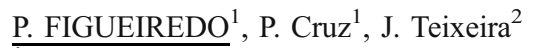

${ }^{1}$ Instituto Superior Tecnico, Lisboa, PORTUGAL, ${ }^{2}$ Sto. António Hospital, Porto, PORTUGAL

\section{PURPOSE}

Mapping the visual cortex is one of the most well established applications of fMRI and could potentially be used to evaluate cortical re-organization in visual field loss disorders or to assist the pre-surgical planning for resection of tumours or epileptogenic tissue. Although fine retinotopic mapping can be achieved with good reproducibility in healthy subjects, the results are dependent on high magnetic field strengths and long acquisitions in order to optimize sensitivity. These conditions are not easily met in a standard clinical setting, where $1.5 \mathrm{~T}$ systems are still common and patient cooperation limits scanning durations. The purpose of this work was to develop a fast visual mapping protocol and to determine its reproducibility.

\section{METHODS}

We implemented a visual field quadrant stimulation paradigm using a flashing checkerboard wedge and tested a group of 5 subjects on two separate sessions. Scanning was performed on a 1.5T Philips system using BOLD imaging and the data were analysed using a standard GLM approach to identify the clusters of activation in primary visual cortex for each quadrant.

\section{RESULTS}

Reproducibility tended to be higher for within- relative to between-session comparisons. Moreover, increasing the dataset size generally increased the reproducibility of the results, as expected. However, no significant systematic differences in reproducibility were found by using different processing parameter combinations. With the optimal processing choices: the median distance between activation peaks was $\sim 3$ to $4 \mathrm{~mm}$; the median percentage of overlapping voxels was $\sim 50$ to $60 \%$; and the median coefficient of variation of the signal change was $\sim 4 \%$.

\section{CONCLUSION}

We have developed and characterized the reproducibility of a fast fMRI protocol for quadrant mapping of retinotopic cortical visual areas. Our reproducibility measurements are comparable to those found in the literature and suggest that this protocol could become sufficiently robust for applications in the clinical routine. 


\section{Cerebrovascular Disease-Imaging and Intervention}

P2:16

\section{THE ROLE OF NONINVASIVE PROCEDURES TCD, CTA, MRA IN DIAGNOSING STENOSIS OF INTRACRANIAL VESELLS}

\author{
V. VUKOVIC ${ }^{1}$, K. Kacar ${ }^{1}$, M. Vukicevic ${ }^{2}$ \\ ${ }^{1}$ Hospital of cerebrovascular diseases "Sveti Sava" Department of \\ neuroradiology., Belgrade, SERBIA, ${ }^{2}$ Hospital of cerebrovascular \\ diseases "Sveti Sava" Department of neurology, Belgrade, SERBIA
}

\section{INTRODUCTION}

Transcranial Doppler (TCD), CT angiography (CTA) and MR angiography (MRA) are noninvasive methods of great importance in estimation of cerebral blood flow (CBF) in patients with symptomatic and asymptomatic stenosis of intracranial vessels. Our main objective is the evaluation of hemodynamic results of noninvasive examinations of patients with suspicious intracranial vessel stenosis.

MATERIAL AND METHODS

29 patients (14 m, 15f), mean age $62 \pm 7$ were examined with application of TCD. 24 of all patients $(83 \%)$ had a brain stroke in the acute/subacute stage of this condition, with symptoms of the affected vascular area, while 5 patients had clinical findings of TIA. TCD was performed using of Spenser technologies PMD (sound of $2 \mathrm{MHz}$ ). MDCT angiography was performed on GE 16 device. Patients with CTA adverse effects or whose clinical findings matched the symptoms of trunci cerebri were examined by 3DTOF MRA sequence (GE 1,5T).

RESULTS

Multifocal stenosis were confirmed with 5 patients (17\%) with the application all three methods. TCD suspicious stenosis was found in the VB territory in 16 patients $(55 \%)$. They were confirmed with CTA, exception two cases. The one of them had stroke of medulla spinalis with restriction of DWI (MRI confirmed) with MRA findings of vertebral artery occlusion. Four patients (14\%) were found TCD suspicious stenosis in the territory of ACM and confirmed by CTA with three patients (in one case CTA was normal). Four patients had TCD suspicious stenosis of ACI (cavernous part), which were confirmed by CTA, except one case which was defined by CTA like occlusion.

CONCLUSION

The most important role has CTA, except in cases with subocclusion where TCD can give additional information, as like MRA and MRI in clinical findings Sy trunci cerebri.

\section{P2:17}

\section{HYPOPLASTIC EXTERNAL CAROTID ARTERY: REPORT OF THREE CASES DIGNOSED BY MRA}

\author{
A. UCHINO, W. MIZUKOSHI \\ Saitama Medical University International Medical Center, \\ Department of Diagnostic Radiology, Hidaka, JAPAN
}

\section{INTRODUCTION}

Agenesis of the main trunk of the external carotid artery (ECA) is rare congenital anomaly. In patients with this anomaly, proximal branches of the ECA, such as lingual, facial, and occipital arteries, arise separately from the distal common carotid artery (CCA).

\section{CASE REPORTS}

Case 1: An 80-year-old man with cerebral infarctions underwent cerebral MRI and MRA including neck region. The 3D-TOFMRA showed high right carotid bifurcation at the level of $\mathrm{C} 3$ vertebral body. The common trunk of the lingual and facial arteries, the distal trunk of the ECA and the occipital artery arise separately from the distal CCA.

Case 2: Cerebral MRI and MRA including neck region were performed in a 48-year-old man with a cerebral infarction. The MRA demonstrated high right carotid bifurcation at the level of C3 vertebral body. From the anomalous trunk of the ECA, the facial artery and the distal trunk arose. The lingual artery arose directly from the distal CCA, and the occipital artery arose from the vertebral artery.

Case 3: A 76-year-old woman with a cerebral infarction underwent cerebral MRI and MRA including neck region. The MRA revealed separately originating lingual, facial, distal trunk of the ECA, and the occipital artery arose from the distal CCA at the level of $\mathrm{C} 3-\mathrm{C} 2$ vertebral bodies.

CONCLUSIONS

Hypoplasitic ECA is rare fusion anomaly of the internal carotid artery and the main trunk of the ECA. In these patients, there is high carotid bifurcation or non-bifurcating carotid artery. Knowledge of this anomaly is important during selective cerebral angiography, interventional procedure, and neck surgery.

\section{P2:18}

\section{CAROTID-ACA ANASTOMOSIS: REPORT OF THREE CASES DIGNOSED BY MRA}

\section{A. UCHINO $^{1}$, Y. OKADA ${ }^{1}$, Y. SATO ${ }^{2}$}

${ }^{1}$ Saitama Medical University International Medical Center, Department of Diagnostic Radiology, Hidaka, JAPAN, ${ }^{2}$ Saitama Medical University, Department of Radiology, Moroyama, JAPAN

\section{INTRODUCTION}

Carotid-ACA anastomosis is rare congenital anomaly. It is also called "ginfraoptic course of ACA" h or "ginteroptic course of ACA" h, because it arises from the ICA at the level of the ophthalmic artery and ascends between the optic nerves.

\section{CASE REPORTS}

Case 1: A 69-year-old woman with syncopal attack underwent cerebral MRI and MRA. The 3D-TOF-MRA showed a large anomalous artery arising from the right ICA at the level of the ophthalmic artery. It ascended within the interoptic space and anastomosed with the right end of the anterior communicating artery. The A1 segment of the right ACA was absent.

Case 2: Cerebral MRI with MRA was performed in a 73-year-old man with cerebral infarctions. The MRA demonstrated an anomalous artery arising from the right ICA at the level of the ophthalmic artery. The A1 segment of the right ACA was present but hypoplastic. The anomalous artery ascended between optic nerves and anastomosed with A1-A2 junction.

Case 3: A 75-year-old man with double vision underwent cerebral MRI and MRA. The MRA revealed bilateral anomalous arteries arising from the ICAs at the level of the ophthalmic arteries. The 
artery of the right side was larger than the other. The A1 segments of the bilateral ACAs were absent.

\section{CONCLUSIONS}

Carotid-ACA anastomosis is rare, but it can be seen unilaterally (right side predominance) or bilaterally. In some cases with this variation, ipsilateral A1 segment is present. Thus, this anomalous artery should not be regarded as an aberrant origin and course of ACA.

\section{P2:19}

\section{PROATLANTAL ARTERY TYPE 2 ASSOCIATED WITH PERSISTENT TRIGEMINAL ARTERY: MR ANGIOGRAPHIC DIAGNOSIS}

\author{
A. UCHINO $^{1}$, K. INOUE $^{1}$, N. KOMIYAMA ${ }^{2}$ \\ ${ }^{1}$ Saitama Medical University International Medical Center, \\ Department of Diagnostic Radiology, Hidaka, JAPAN, ${ }^{2}$ Saitama \\ Medical University International Medical Center, Department of \\ Cardiovascular Medicine, Hidaka, JAPAN
}

\section{INTRODUCTION}

Proatlantal artery type 2 is extremely rare congenital anastomosis between the proximal external carotid artery (ECA) and the vertebral artery (VA) via an extremely dilated proximal occipital artery (OA).

CASE REPORT

An 83-year-old man with cerebral infarctions underwent cerebral MRI and head \& neck MRA. The 3D-TOF-MRA showed aplasia of the proximal left VA, hypoplasia of the right VA, and a large anastomotic artery between the left ECA and the distal left VA at the level of the foramen magnum. From this anastomotic artery, distal branch of the OA arose. Thus, we diagnosed that this was the proatlantal artery type 2 . This patient also had an anastomosis between the precavernous segment of the left internal carotid artery and the midbasilar artery, indicative of the persistent trigeminal artery, the most frequently observed type of the carotid-vertebrobasilar anastomoses. This is, to our knowledge, the first reported case of such association.

CONCLUSIONS

We reported the first case of the proatlantal artery type 2 associated with the ipsilateral trigeminal artery diagnosed by MR angiography. If cerebral MR angiography is routinely performed including the carotid bifurcation, extracranial arterial variations including the proatlantal artery type 2 may be found more frequently.

\section{P2:20}

\section{STENTING FOR THE TREATMENT OF INTRACRANIAL STENOSIS}

N. TSAMOPOULOS $^{1}{ }^{1}$ M. PAPATHANASIOU $^{2}$, L. VALVASSORI $^{1}$, E. BOCCARDI ${ }^{1}$, A. GOULIAMOS ${ }^{2}$

${ }^{1}$ Ospedale Niguarda $\mathrm{Ca}$ 'Granda - Department of Interventional and Therapeutic Neuroradiology, MILANO, ITALY, ${ }^{2}$ National and Kapodistrian University of Athens - "ATTIKON" University Hospital - Department of Radiology, ATHENS, GREECE

\section{PURPOSE}

To evaluate the safety, the clinical efficacy and the angiographic outcome of stenting for patients with symptomatic intracranial stenosis. And also, to determine the significance of classification based on location, morphology and access of stenosis in intracranial stenting. METHODS

We evaluated the outcome of primary stenting of 14 of our patients, with 15 intracranial stenosis, while on antithrombotic therapy. All patients had neurologic symptoms ( ischemic stroke or transient ischemic attack ) attributable to intracranial stenosis $>80 \%$. The lesions were situated in the Left intracranial Internal Carotid artery $(n=03)$, Right intracranial Internal Carotid artery $(n=03)$, Left Vertebral artery $(n=02)$, Right Vertebral Artery $(n=01)$, VertebralBasilar junction $(n=01)$ and Basilar artery $(n=05)$. They were classified according to Location-Morphology-Access (LMA) classification $\}$ morphologically into Type $A(n=08)$, Type $B(n=06)$ and Type C $(n=01)$ lesions. And into Type I $(n=05)$, Type II $(n=02)$ and Type III $(n=08)$ accesses. Patients age ranged from 55 to 89 years. RESULTS

The technical success rate was $100 \%$ for total lesions. There was only one complication, without consequences - in one case a dissection occured which was successfully managed by deploying a stent — giving an overall complication rate of $7 \%$ ( $1 / 14$ patients). The clinical outcome was good, according to Modified Rankin Scale (MRS) was 0-1. CONCLUSIONS

Stenting for the treatment of intracranial stenosis appears to be an effective and feasible therapy with a high degree of procedural success and relatively low risk of complications. A classification based on location, morphology and access of stenosis seems to be helpful to decide on the treatment for the individual patient, to predict the prognosis of stenting and conclude the procedure in the shortest time with the least complications.

\section{P2:21}

\section{VERTEBROBASILAR ARTERY DISSECTION IN ACUTE CEREBELLAR AND/OR BRAIN STEM INFARCTION}

\section{Y. TOYOGUCHI, A. ODA, T. HOSOYA, M. KANOTO \\ DEPT. OF DIAGNOSTIC RADIOLOGY, FACULTY OF MEDICINE, YAMAGATA UNIVERSITY, YAMAGATA, JAPAN}

\section{PURPOSE}

To clarify the relationship between vertebrobasilar artery dissection and acute cerebellar and/or brain stem infarctions using basiparallel anatomical scanning (BPAS) as a screening method. SUBJECTS AND METHODS

We investigated 25 patients (18 men, 7 women) aged 35 to 87 years (mean, 70.7 years) having acute cerebellar and/or brain stem infarctions without cerebral infarctions. We performed MR angiography (MRA) and BPAS addition to routine MRI examination in all patients. If possible, we performed further neuroradiological examinations; such as contrast-enhanced T1FFE, contrast-enhanced CT, and DSA.

RESULTS

Among 25 patients, 14 showed external dilatation of vertebrobasilar artery on BPAS. Of 15 patients with contrast-enhanced T1FFE, contrast-enhanced CT, and/or angiography, 9 patients were diagnosed as vertebrobasilar artery dissection. Of the 9 patients diagnosed as vertebrobasilar artery dissection, 8 patients showed external dilatation of vertebrobasilar artery on BPAS. 


\section{CONCLUSIONS}

Vertebrobasilar artery dissection frequently occurred in the patients with acute cerebellar and/or brain stem infarction. Combination of MRA and BPAS is useful screening method for vertebrobasilar artery dissection.

\section{P2:22}

\section{PROGRESSIVE ADC VALUES (DWI) IN ISCHEMIC STROKE}

S. Benakis ${ }^{1}$, C. Louizakis ${ }^{2}$, D. Exarhos ${ }^{1}$, V. Ouranos ${ }^{1}$, K. Stefanidis ${ }^{1}$, $\overline{\text { I. Skouras }}^{1}$, V. Vantali ${ }^{1}$, A. Pavlopoulou ${ }^{1}$, D. Chondros ${ }^{1}$

${ }^{1}$ Evangelismos Hospital, CT \& MRI Department, Athens, GREECE,

${ }^{2}$ Polikliniki Hospital, Department of Radiology, Athens, GREECE

\section{PURPOSE}

To investigate DWI, ADC and Expo-ADC values at the various stages of ischemic stroke, beyond hyperacute stage, according to the transition of cytotoxic to vasogenic brain edema and at last to gliosis and neuronal loss of the diseased area. To evaluate the usefulness of DWI and calculation of ADC and Expo-ADC values in definition of the stage of a stroke in cases of undeterminated medical history.

METHODS

26 patients, with symptoms of ischemic stroke of various time of appearance, were examined and retrospectively analyzed at the CTMRI department. According to the time interval between symptoms initiation and MRI examination, four stages were determined: acute $(n=3)$, early subacute $(n=7)$, late subacute $(n=9)$, and chronic $(n=7)$. RESULTS

DWI showed increased signal intensity in all acute and subacute stages of ischemic stroke, except from chronic stage of gliosis that showed hypointensity. ADC maps and values were calculated in the specific areas showing low values at acute and early subacute stage (small amount of vasogenic edema), iso- to hyperintense at late subacute stage (increased amount of vasogenic edema), and increased ADC value at neuronal loss of chronic stages. Exponential ADC values appeared to be hyperintense at the first stages and iso- to hypointense at late subacute, in correlation with ADC maps.

CONCLUSIONS

In spite its well established usefulness at hyperacute ischemic strokes in the first 6 hours, DWI, ADC and Exponential ADC map might be helpful in the determination of other stages of ischemic strokes, especially in differentiation of the early and late subacute stage following the variance of the amount of cytotoxic and vasogenic edema.

\section{P2:23}

\section{ADC VALUES AND CONVENTIONAL T2* GRADIENT-ECHO SEQUENCE IN BRAIN HEMORRHAGE}

D. Exarhos ${ }^{1}$, C. Louizakis $^{2}$, V. Ouranos ${ }^{1}$, K. Stefanidis ${ }^{1}$, I. Skouras ${ }^{1}, \underline{\text { S. Benakis }}{ }^{1}$, A. Skoula ${ }^{1}$, S. Tsekouras ${ }^{1}$, D. Chondros ${ }^{1}$ ${ }^{1}$ Evangelismos Hospital, CT \& MRI Department, Athens, GREECE, ${ }^{2}$ Polikliniki Hospital, Department of Radiology, Athens, GREECE

\section{PURPOSE}

To investigate DWI sequences and calculate ADC and Expo-ADC values at the various stages of a brain hemorrhage relatively to $\mathrm{T} 2 *$ sequence. To evaluate the usefulness of its sequence in the determination of the stage of the process.

METHODS

In this study, 16 patients with hemorrhage underwent an MRI protocol that included diffusion weighted imaging (DWI) and T2* gradientecho images in 12 of them. We investigated the signal intensity and apparent diffusion coefficient (ADC) and compared the signal intensities of hemorrhage in DWI and T2* gradient echo MR images. The analysis included patients with symptoms of stroke of various time of appearance, categorized in four stages: acute $(n=2)$, early subacute $(n=6)$, late subacute $(n=5)$, and chronic $(n=3)$, examined at the CT-MRI department.

RESULTS

Brain hemorrhage in DWI showed hypointense at the acute and early subacute stage, hyperintense at late subacute and iso- or hyperintense at chronic stages. The ADC values at the acute and early subacute stage were hypointense relatively to normal white and grey matter, and at late subacute were of variable signal ranged from hypointense to medium hyperintense, and hyperintense at the chronic stage. T2* sequence showed hypointense at acute and early subacute stage, hyperintense at late subacute and hypo- or isointense surrounded by hypointense rim at chronic stage.

CONCLUSION

MRI protocols including DWI, ADC maps and conventional T2* sequence additional to common MR sequences may be useful in determination of the stage of hemorrhagic strokes, and in the DD between hemorrhagic and ischemic stroke, especially in subacute stages, and other brain pathology.

\section{P2:24}

\section{ANTIPHOSPHOLIPID ANTIBODY SYNDROME: CT AND MR FINDINGS OF THE BRAIN}

\author{
V. Vantali ${ }^{1}$, V. Ouranos ${ }^{1}$, C. Louizakis ${ }^{2}$, K. Stefanidis ${ }^{1}, \underline{\text { S. Benakis }}{ }^{1}$, \\ D. Exarhos ${ }^{1}$, E. Kratimenou ${ }^{1}$, N. Ananiadis ${ }^{1}$, D. Chondros ${ }^{1}$ \\ ${ }^{1}$ Evangelismos Hospital, CT \& MRI Department, Athens, \\ GREECE, ${ }^{2}$ Polikliniki Hospital, Department of Radiology, Athens, \\ GREECE
}

\section{PURPOSE}

The antiphospholipid syndrome (APS) is an autoimmune prothrombotic condition characterized by arterial and/or venous thrombosis and pregnancy morbidity in the presence of antiphospholipid antibodies. The purpose of this study was to determine the spectrum of neuroradiologic findings of the brain in patients with APS. METHODS

We retrospectively analyzed 8 patients (mean age 29 y.o. range: 19 38 y.o.) with antiphospholipid antibody syndrome who underwent CT or MR imaging. In five patients APS presented in association with systemic lupus erythematosus (secondary APS), and in three patients as an isolated disorder (primary APS). All patients initially presented with neurologic symptoms without any other systemic manifestations. A standard MRI protocol with intravenous contrast media was performed with additional diffusion weighted imaging (DWI) in all patients. 


\section{RESULTS}

The CT or MRI findings were normal in 2 patients. The initial involvement was cerebral in 5 cases and cerebellar in one case. Multiinfarct atrophy was seen in two patients with dementia. Large infarcts were seen in 5 patients. One patient with large cerebellar and cerebral infarcts, complicated with hydrocephalus, showed hemorrhagic transformation. Small cortical infarcts and lacunar infarcts were depicted in two patients. T2-weighted images revealed multiple focal areas of increased signal intensity in white matter, in gray/white matter junction, in both cerebellar hemispheres, in cerebellar peduncles and in thalamus. Patients with hyperintense white matter foci had at least one large lesion, and two patients had five or more small foci. One patient with large white matter hyperintense lesions had also prominent welldefined area of increased signal intensity in corpus callosum that could be presumed to represent ischemic lesions of the brain.

CONCLUSION

Usually large Infarcts and hyperintense white matter foci are the most common abnormalities seen on CT and MRI. Radiologists should always consider the possibility of APL syndrome when the above type of lesions are seen.

P2:25

\section{VERTEBRAL ARTERY-TO-POSTERIOR INFERIOR CEREBELLAR ARTERY STENTING FOR PRESERVATION OF THE PICA IN THE RUPTURED VERTEBRAL ARTERY DISSECTION}

Y. SHIN, J. Chung, D. Lee, B. Kim

St Mary's Hospital, Catholic University of Korea, Seoul, SOUTH KOREA

The ideal method of treatment for patients with vertebral artery (VA) dissections involving the posterior inferior cerebellar artery (PICA) origin is complete isolation of the dissected segment by trapping, with revascularization of the PICA. Instead, the authors reported a patient treated by placing VA-to-PICA stenting to save the PICA patency and internal trapping of the dissected segment by coiling. A 47-year-old man was admitted with Hunt-Hess (HH) Grade II SAH. Angiography revealed a right VA dissecting aneurysm on involving the origin of the PICA. Left VA was patent. In order to saving PICA flow, we placed the Enterprise stent (Cordis Neurovascular, Miami Lakes, FL, USA) through proximal VA to PICA. Then, coil embolization of the dissected segment was performed. Three-month follow-up angiography showed that the dissected segment was completely occluded and the diameter of the PICA was increased with a good stent patency.

\section{P2:26}

\section{HEMORRHAGE IN THE NORMOTENSIVE ELDERLY: THE FACES OF AMYLOID ANGIOPATHY}

I. SARALEGUI PRIETO, J.M. ONTAÑON GARCES, A. CABRERA ZUBIZARRETA, I. VICENTE OLABARRIA, B. FERNANDEZ RUANOVA

GALDAKAO HOSPITAL OSATEK S.A. MR UNIT, GALDAKAO-BILBAO, SPAIN

\section{PURPOSE}

To describe both the typical and atypical features of cerebral amyloid angiopathy (CAA) on MRI and to review the imaging protocol to reach a correct diagnosis.

METHOD

Since 2001 we retrospectively reviewed the MRI findings of 200 patients with a probable or probable with supporting pathologic evidence cerebral amyloid angiopathy based on the Boston criteria. Apart from the standard cranial protocol, T2 gradientecho and more recently susceptibility-weighted imaging were sistematically performed.

RESULTS

CAA is an important but underrecognized cause of cerebrovascular disorders that predominantly affects elderly patients. CAA results from deposition of $\beta$ amyloid protein within the cerebral arterioles, which is responsible for the wide spectrum of clinical symptoms and neuroimaging findings. Currently, CAA can only be diagnosed histopathologically following biopsy or at postmortem examination. Many cases of CAA are asymptomatic. Magnetic Resonance Imaging is an important tool for the diagnosis of these patients, exclusion of other causes of acute cortical-subcortical hemorrhage and assessment of disease progression. Most of the times a correct diagnosis of CAA is easily done based on MRI because of visualization of the typical findings of cortical-subcortical microhemorrhages or other types of lobar, subarachnoid or subdural hemorrhages. Nevertheless, It's important to recognize other less common signs described in some cases of CAA even in the absence of cerebral hemorrhages, such as atrophy, ischemic infarcts, tumoral deposition of amyloid (amyloidoma) or leukoencephalopathy, both including and sparing $U$ fibers (reversible).

Conventional gradient-echo imaging is a well-established technique for detecting cerebral microhemorrhages, although has been recently substituted by a new neuroimaging technique, susceptibility-weighted imaging, based on tissue magnetic susceptibility differences to generate a unique contrast, being more detailed in identifying the microangiopathic changes seen in CAA and a more sensitive diagnostic tool. CONCLUSIONS

Accurate recognition of imaging findings is important in guiding clinical decision making in patients with CAA.

P2:27

\section{CLAUDE'S SYNDROME WITHOUT INVOLVEMENT OF THE RED NUCLEUS}

\author{
G. SANTOS $^{1}$, S. PEREIRA ${ }^{1}$, J. FREITAS ${ }^{2}$, G. CORDEIRO ${ }^{2}$, \\ E. MACHADO ${ }^{1}$ \\ ${ }^{1}$ Hospitais da Universidade de Coimbra - Neuroradiology Unit - \\ University Clinic of Imagiology, Coimbra, PORTUGAL, ${ }^{2}$ Hospitais \\ da Universidade de Coimbra - Department of Neurology, Coimbra, \\ PORTUGAL
}

\section{BACKGROUND AND PURPOSE}

Claude's syndrome represents a midbrain vascular syndrome characterized by homolateral oculomotor nerve palsy and contralateral cerebellar ataxia. Although classically attributed to involvement of the red nucleus, there is still some discussion regarding lesion location in Claude's syndrome. Superior cerebellar peduncle has been proposed as the probable lesion site. 
The authors present a case of Claude's syndrome without involvement of the red nucleus.

\section{METHODS AND RESULTS}

A 61 year old male patient presented to the emergency department with sudden horizontal diplopia. He had a past history of type 2 diabetes mellitus and hypertension.

On neurological examination, a complete palsy of the right oculomotor nerve was noted. Also, the patient had left kinetic ataxia and ataxic gait with veering to the left.

Head CT showed no recent ischemic or hemorrhagic lesions. CT angiography showed occlusion of the right internal carotid artery and vertebrobasilar atheromatous dolichoectasia.

Patient then underwent MR imaging that showed an acute ischemic lesion involving the tegmentum of the midbrain, medial and inferior to the right red nucleus, and involving fibers from the superior cerebellar peduncle.

CONCLUSIONS

This case seems to support the hypothesis that the red nucleus is not the lesion site in Claude's syndrome.

\section{P2:28}

\section{VARIANTS OF THE CAUDAL DIVISION OF THE INTERNAL CAROTID ARTERY AND OF THE BASILAR ARTERY DEMONSTRATED BY CT ANGIOGRAPHY: A PICTORIAL ESSAY}

\author{
G. SANTOS, C. MARQUES, S. PEREIRA, T. PARREIRA, \\ F. RIO, E. MACHADO \\ Hospitais da Universidade de Coimbra - Neuroradiology Unit - \\ Department of Imagiology, Coimbra, PORTUGAL
}

\section{PURPOSE}

Cerebral CT-Angiography (CTA) is a non invasive technique that allows acquisition of images of high spatial resolution with short aquisition time. Besides being a first-line method for detecting vascular pathology, it can accurately display the vascular anatomy and its variations.

Posterior communicating artery and $\mathrm{P} 1$ segment of the posterior cerebral artery (PCA) constitute the caudal division of the internal carotid artery (ICA), communicating with the tip of the basilar artery. These vessels are subject to anatomical variants.

While most of these variants have no major clinical significance, their recognition can be important to the interventional neuroradiologist and the neurosurgeon in order to decide the appropriate therapy.

We present a review of the normal anatomy and variants of the caudal division of the ICA and the basilar artery, as detected on Cerebral CTA, discussing aspects relevant to clinical practice.

METHODS

Retrospective review of the cerebral CTAs performed at our institution in 2008, on a 64 detector row CT scanner. Patients with history of cerebral vascular surgery were excluded. The normal anatomy and variants are demonstrated in $3 \mathrm{D}$ reconstructions.

\section{RESULTS}

Among the 68 evaluated exams we found vascular pathology in $51.5 \%$ of patients (aneurysms in $29.4 \%$ ). Fetal origin of the PCA was the most frequent variant (39.7\%). Fenestrations of the basilar artery were present in $11.8 \%$ of cases. In $48.5 \%$ of patients there was a basilar tip with a cranial fusion disposition. P1 asymmetry was noted in $27.9 \%$ of patients.

CONCLUSIONS

Our results confirmed the anatomical variability of the caudal division of the ICA and the basilar artery. We found a high frequency of fetal origin of the PCA and of basilar fenestrations compared to the literature, which can be related to the high frequency of vascular pathology in our population.

Cerebral CTA was efficient in demonstrating the vascular anatomy.

\section{P2:29}

\section{CEREBRAL VENOUS THROMBOSIS: AN UPDATE}

$\underline{\text { J. SANCHEZ }}{ }^{1}$, P. PUYALTO ${ }^{2}$, A. CAMINS ${ }^{1}$, P. CUADRAS ${ }^{2}$, C. AGUILERA ${ }^{1}$

${ }^{1}$ IDI-Hospital de Bellvitge-Duran i Reynalds, Neuroradiology Department, Barcelona, SPAIN, ${ }^{2}$ Hospital Germans Trias i Pujol, Neuroradiology Department, Barcelona, SPAIN

\section{PURPOSE}

The aims of this exhibit are:

1) To review the cerebral venous normal anatomy and most common variants.

2) To describe the etiological factors and physiopathology of this disorder.

3) To present the radiographic findings and pitfalls of cerebral venous thrombosis.

4) To review the imaging modalities and techniques protocols used in the diagnosis of this entity.

\section{METHODS}

47 patients with cerebral venous trombosis were retrospectively reviewed by two experienced neuroradiologists. Imaging techniques such as Unenhanced Computer Tomography (CT), CT-venography , Conventional Magnetic Resonance (MR) and MR-venography and theirs imaging protocols were reviewed. Some common and uncommon pitfalls were also assessed.

\section{RESULTS}

Cerebral venous thrombosis is a serious neurological disorder with a mortality range of $10-80 \%$ that is potentially reversible with a appropriate medical care. Haemostatic disorders and pro-thrombotic drugs are the leading causes of cerebral venous thrombosis. MR with angiographic sequences (venography) can show the presence of a thrombus within a venous vessel and its consequences on the cerebral parenchyma such as haemorrhagic infarction following an atypical vascular territory distribution.

\section{CONCLUSIONS}

MR- venography in conjunction with conventional MR can accurately diagnose cerebral venous thrombosis. With careful interpretation and a high degree of clinical suspicion, CT also may lead to the diagnosis, although pitfalls must be avoid. 


\section{P2:30}

\section{INTRACRANIAL AND EXTRACRANIAL ATHEROSCLEROSIS: THE SAME DISEASE? ANGIOGRAPHIC ANALYSIS OF A MEDITERRANEAN POPULATION}

\section{J. RAMALHO, I. FRAGATA, J. REIS}

Centro Hospitalar de Lisboa Central, EPE; Department of Neurorradiology, Lisbon, PORTUGAL

\section{PURPOSE}

It has been demonstrated that there is a high association of extracranial (EC) and intracranial (IC) atherosclerotic disease in certain populations. We evaluated the prevalence of IC and EC atherosclerosis in a Mediterranean group of patients with ischemic stroke, to determine the relations between IC and EC stenosis and atherosclerotic risk factors.

\section{METHODS AND MATERIALS}

We retrospectively reviewed the digital subtraction angiography findings and charts of 103 consecutive ischemic stroke patients with intracranial and/or extracranial atherosclerosis (defined as $>$ or $=30 \%$ narrowing of the luminal diameter), that underwent digital subtraction angiography, focusing on the location of stenosis, and comparison of risk factors between groups.

\section{RESULTS}

A total number of 103 patients was studied, with $59.5 \%$ isolated EC atherosclerosis, 31\% isolated IC atherosclerosis, and only 9.5\% combined IC and EC disease. Patients in the IC atherosclerosis group were significantly younger (56.5 versus 65.8 years), had a higher incidence of diabetes mellitus (53.8\% versus $28 \%$ ), and less smoking habits (15.4\% versus $40 \%)$.

\section{CONCLUSION}

In our population, there seems to be poor association between IC and EC stenosis, and different associated risk factors for each location. Careful evaluation of the intracranial circulation in young, diabetic ischemic stroke patients should be performed, even in the absence of EC atherosclerotic disease.

\section{P2:31}

\section{COMPUTED TOMOGRAPHY ANGIOGRAPHY ROLE IN CAROTID STENOSIS}

T. PARREIRA, C. MARQUES, C. CASIMIRO, P. LIMA, S. PEREIRA, P. MELO FREITAS

Hospitais da Universidade de Coimbra - Clinica Universitária de Imagiologia - Neurorradiologia, Coimbra, PORTUGAL

\section{PURPOSE}

To characterize the role of computed tomography angiography (CTA) evaluating the stenosis degree and the composition of atherosclerotic plaques, correlating with the presence of neurologic symptoms. We also evaluate the agreement between ultrasound echo-colour doppler (US-ECD) and CTA determining the stenosis degree and characterizing plaque composition.

\section{METHODS}

From April 2007 to December 2008, 138 patients who had previously undergone CTA were studied retrospectively. For each patient, stenosis degree applying the North American Symptomatic Carotid Trial (NASCET) criteria, plaque composition and distribution, anatomical variants and neurologic symptoms was obtained. When available, data from the USECD relative to the stenosis degree and plaque composition was obtained. Patients with non-atheromatous carotid stenosis were excluded.

RESULTS

$52 \%$ of the CTA observed plaques were located at the carotid bulb and $31 \%$ at the bifurcation. Plaques showing sole calcium component at CTA correlate with asymptomatic neurological status and purely lipidic plaques at CTA correlate with the presence of ipsilateral neurological symptoms $(p=0.008$, Odds Ratio=5). Plaque ulceration at CTA strongly correlates with the presence of neurological symptoms (left: $p=0.026$ / right: $p=$ 0.012). The stenosis degree shows good agreement between the two evaluated techniques (Pearson correlation $\mathrm{p}<0.01 \mathrm{k}=0.7$ ). The stenosis degree using the minimum vessel diameter correlates, as expected, with stenosis degree using the vessel area, scatterplot showing higher values of area stenosis. The mean diameter stenosis within the symptomatic group is higher and statistically significant comparing to the asymptomatic group.

\section{CONCLUSION}

CTA is a useful technique determining the stenosis degree, composition of atherosclerotic plaques and detecting plaque ulceration, factors related to neurological symptoms. Threedimensional CT reconstructions offer a unique opportunity for surgeons to visualize the carotid plaque and surrounding structures prior to endarterectomy.

P2:32

\section{SENSITIVITY OF MAGNITUDE SUSCEPTIBILITY WEIGHTED IMAGING (SWIM) IN DETECTING FOCAL CEREBRAL HEMORRHAGIC LESIONS}

J. MUNUERA DEL CERRO, R. Mitjana, S. Mecho, S. Siurana, S. Sarria, A. Rovira

Magnetic Resonance Unit, Department of Radiology (IDI), Hospital Universitario Vall d'Hebron, Barcelona, SPAIN

\section{OBJECTIVE}

To analyze the sensitivity of SWIm in detecting focal brain hemorrhagic lesions, as compared to 2 other T2 sequences: turbo spin echo (T2 TSE) and gradient echo (T2* GE).

MATERIAL AND METHOD

Over an 8-month period, a prospective brain MRI study was performed in a cohort of 62 patients with a clinical diagnosis of stroke, chronic cerebrovascular disease, vascular malformation, brain trauma, or brain tumor. The number of focal hemorrhagic 
lesions seen in the 3 sequences (T2 turbo spin echo, T2* gradient echo, and SWIm) was assessed by 2 radiologists, first independently and then by consensus.

SWIm images were obtained on a 1.5-T magnet, based on a short TR gradient-echo 3D sequence. The volumetric images were postprocessed using a minimum intensity projection algorithm (minIP), with the same section position and thickness as the other T2 sequences.

\section{RESULTS}

Focal brain hemorrhages were identified in 40 of $62(65 \%)$ patients (mean age, 58 years). Mean number of lesions detected was 31 (range, 0-500) on SWIm, 16 (range, 0-300) on T2* gradient-echo, and 8 (range, 0-8) on T2 turbo spin-echo sequences. Statistical analysis showed that SWIm was significantly more sensitive $(\mathrm{P}<0.005)$ than the other $\mathrm{T} 2 \mathrm{~W}$ sequences studied, and confirmed the higher sensitivity of $\mathrm{T} 2 *$ gradientecho compared with $\mathrm{T} 2 \mathrm{~W}$ turbo spin-echo sequences.

\section{CONCLUSION}

The acquisition properties and post-processing of the SWIm sequence result in significantly higher sensitivity of MRI for detecting focal brain hemorrhagic lesions.

\section{P2:33}

\section{VALUE OF 64 ROW CT ANGIOGRAPHY (CTA) WITH IODINE LOW DOSE INJECTION IN THE MANAGEMENT OF THE NON-TRAUMATIC SUBARACHNOID HEMORRHAGE}

\section{MILLON, A.L. DERELLE, P.A. LEBEDINSKI, V. DELMIRO, R. ANXIONNAT, S. BRACARD \\ Hôpital Central-Service de Neuroradiologie, NANCY, FRANCE}

\section{PURPOSE}

The aim of this study is to evaluate the CTA protocol on a 64 multidetector row spiral CT, with a low dose contrast agent injection, in the management of non-traumatic subarachnoid haemorrhage $(\mathrm{SAH})$.

METHODS

We retrospectively studied 73 patients who presented SAH, from August 2007 to September 2008.

CTA was performed in emergency on a 64 multidetector row spiral CT (GEMS), with automatic injection of $25 \mathrm{~mL}$ of iomeprol $($ Bracco) $(5 \mathrm{~mL} / \mathrm{s})$, followed by $25 \mathrm{~mL}$ of saline.

RESULTS

73 patients were included. In 3 cases, CTA findings were inconclusive, and digital subtraction angiography (DSA) has been performed to get further information. In these cases, despite successive acquisitions, poor vessels opacification was due to intra cranial hyperpressure.

CTA was sufficient to manage the remaining 70 patients

In 57 cases, CTA was positive, and could diagnose the origin of the haemorrhage (52 aneurysms, 2 AVM, 1DAVF and 2 dissections).

No vascular abnormality was diagnosed on the 13 other patients. $2 / 13$ patients died before the angiographic evaluation. The lack of vascular abnormality was confirmed by the DSA in the remaining 11 cases.

All associated aneurysms, except one were diagnosed on CTA (C1 segment of internal carotid artery, $2 \mathrm{~mm}$ length)

Morphologic evaluation of aneurysms was considered as sufficient to make the therapeutic choice. 10 were clipped and 44 were coiled.

\section{CONCLUSION}

CTA, with low dose injection should be used as the first diagnostic modality for patients with non-traumatic subarachnoid haemorrhage.

It represents a very good test to manage and select correct patient for any therapy.

\section{P2:34}

\section{ANATOMIC VARIANTS OF A1-ACOA-A2 JUNCTION IN BRAIN CT-ANGIOGRAPHY-A PICTORIAL ESSAY}

C. MARQUES, G. Santos, T. Parreira, S. Pereira, P. Bastos Lima, E. Machado, F. Rio

Neuroradiology Unit, Department of Imagiology, Hospitais da Universidade de Coimbra, Coimbra, PORTUGAL

\section{BACKGROUND AND PURPOSE}

The circle of Willis constitutes a vital anastomotic vascular ring, providing collateral cerebral blood circulation in cases of occlusive vascular disease. This potential collateral pathway depends on the circle's morphology, which is known to be extremely variable. In addition, there are anatomic anomalies, which are associated with an increased prevalence of intracranial aneurysms. CT-angiography is a noninvasive modality that plays an important role in detecting the intra-cranial vascular anatomy and pathology, because of its high resolution imaging and fast acquisition time. The purpose of this study is to evaluate the efficiency of CT-angiography (CTA) in characterizing, through a pictorial essay, the anatomy of the junction between the anterior communicating artery and the first and second segments of the anterior cerebral arteries, (A1-ACoA$\mathrm{A} 2$ ), in order to recognize the most frequent anatomic variants.

\section{MATERIALS AND METHODS}

Retrospective review of the cerebral CTAs performed at our institution in 2008, on a 64 detector row CT scanner. Patients with history of cerebral vascular surgery were excluded. The normal anatomy and variants are demonstrated in $3 \mathrm{D}$ reconstructions.

\section{RESULTS}

68 CTAs were evaluated. Brain haemorrhage was the most frequent reason for performing the exam. Vascular disease was found in $51.5 \%$ of patients (aneurysms in 29.4\%). In what concerns anatomic variability, the calibre of A1 segments was found to be asymmetric in $17.6 \%$ of the cases. Fenestration constituted the most frequent anatomic variant affecting the anterior communicating artery $(13.2 \%)$. The persistence of the embryonic median artery of the corpus callosum was identified in 
$2.9 \%$ of the patients and a bihemispheric anterior cerebral artery was present in $4.4 \%$.

\section{CONCLUSION}

The data obtained confirmed the anatomic variability in the anterior part of the circle of Willis, agreeing generally with the values referred by literature. CT-angiography clearly demonstrates the circle's morphology, allowing an efficient illustrative anatomic characterization.

\section{P2:35}

\section{UNRECOGNIZED EARLY SUBTLE CT SIGN OF VENOUS CEREBRAL INFARCTION WITH CONSEQUENT LETHAL OUTCOME}

\author{
D. KOZIC $^{1}$, B. Radovanovic ${ }^{2}$, D. Ivanovski ${ }^{2}$, M. Zarkov ${ }^{2}$ \\ ${ }_{1}^{1}$ Institute of Oncology, Diagnostic Imaging Center, Sremska \\ Kamenica, SERBIA, ${ }^{2}$ Clinical Center of Voivodina, Clinic of \\ Neurology, Novi Sad, SERBIA
}

\section{PURPOSE}

To point out the early CT sign of venous infarct that was missed on initial scannining.

\section{METHODS}

Initial brain $\mathrm{CT}$ examination followed by $\mathrm{CT}$ and $\mathrm{MR}$ brain scanning three and four days later was performed.

\section{RESULTS}

CT examination of the brain was indicated in a normotensive, afebrile patient with a two days history of nausea, vomiting, worsening neck pain and occipital headache. No abnormalities were noted on brain $\mathrm{CT}$ and the patient was discharged with recommendation for routine laboratory tests and plain X-ray of the cervical spine. However, right hemiparesis was evident next day with persistant headache. The patient was sent back to Neurology Clinic where he developed myoclonic seizure compatible with focal motor status epilepticus, just after the lumbar punture. The therapy for status epilepticus was administred and the emergency MRI with angiography was indicated. The another status epilepticus appered within the MR scanner and the exam was discontinued. Glasgow coma scale was 3. Leucocytosis withe neutrophilia were found on routine laboratory study. The Glasgow coma scale worsened to 6 witin 48 hours. CT and MR examination of the brain revealed three huge hemorrhagic venous infarcts in the left cerebral hemisphere associated with the typical signs of venous thrombosis. The presence of subtle curvilinear hyperdensity was detected in the left parietal cortical-subcortical border during the reevaluation of initial brain $\mathrm{CT}$, most compatible with developing venous infarct. No improvement was noted after administration of anticoagulant treatment and the lethal outcome appeared 11 days after initial CT scanning.

\section{CONCLUSION}

Detection of early parenchimal signs of venous throbosis is extremely important, especially in patients with vague delta sign, since the delay of adequate tretment may have catastrophic consequences.
P2:36

\section{NEUROIMAGING EVALUATION OF "TOP OF THE BASILAR" SYNDROME}

Y. KIROGLU ${ }^{1}$, C. ONCEL ${ }^{2}$, B. CIRAK ${ }^{3}$, I. ORAN ${ }^{4}$, N. KARABULUT ${ }^{5}$, S. SENEL ${ }^{6}$, S. AKALIN ${ }^{7}$, I. AKDOGAN ${ }^{8}$

${ }^{1}$ Pamukkale University School of Medicine-Depatment of Radiology, Denizli, TURKEY, ${ }^{2}$ Pamukkale University School of MedicineDepartment of Neurology, Denizli, TURKEY, ${ }^{3}$ Pamukkale University School of Medicine-Department of neurosurgery, Denizli, TURKEY, ${ }^{4}$ Ege University School of Medicine-Department of Radiology, Izmir, TURKEY, ${ }^{5}$ Pamukkale University School of Medicine-Department of Radiology, Denizli, TURKEY, ${ }^{6}$ Pamukkale University School of Medicine-Department of Internal Medicine, Denizli, TURKEY, ${ }^{7}$ Pamukkale University School of Medicine-Department of Infectious diseases, Denizli, TURKEY, ${ }^{8}$ Pamukkale University School of Medicine-Department of Anatomy, Denizli, TURKEY

Abstract

PURPOSE

To evaluate the imaging manifestations and clinical findings of "Top of the basilar" syndrome (TOB-S).

METHOD

We retrospectively evaluated the clinical and imaging features of six patients who had distal basilar artery (BA) circulatory disturbance using magnetic resonance imaging (MRI), computed tomography (CT) and digital subtraction angiography (DSA). RESULTS

Six patients had diagnosis of TOB-S based on the neuroimaging and clinical findings. The underlying cause was atherosclerosis of vertebrobasilar system (VBS) in two, thromboembolus in two, chronic vasculitis in one and traumatic dissection in one patient. Corresponding of ischemic areas in the rostral BA territory was demonstrated in thalamus, medial temporal and occipital lobes, cerebellum and brain stem.

CONCLUSION

Familiarity with the pattern and distribution of infarcts, and imaging features of distal BA territories help accurate diagnosis of TOB-S.

P2:37

\section{LONG TERM FOLLOW UP OF NON-HEMORRHAGIC VERTEBROBASILAR ARTERY DISSECTION AFTER STENTING}

S. KIM, H.S. KIM, N.J. LIM

Ajou University Hospital, Department of Radiology, Suwon, SOUTH KOREA

\section{PURPOSE}

The therapeutic management of patients presenting with nonhemorrhagic vertebrobasilar dissection remains controversial. Currently, few longterm follow up after stent placement are available, We evaluated the effectiveness of stent placement for vertebrobasilar dissection by re-evaluated angiographic and clinical results. 


\section{MATERIALS AND METHODS}

We reviewed 13 patients ( 9 men, 4 women; age range 36 to 46 years) during the last 5 years, with nonhemorrhagic vertebrobasilar dissection, were treated by stenting. 11 lesions were located at vertebral artery( two bilateral cases), and two are basilar artery. 10 patients presented ischemic symptoms and 3 with severe headache. Initial angiographic findings included abrupt artery narrowing with aneurismal dilatations in 9 lesions and irregular bulubous aneurismal dilatation in 4 lesions.

8 lesions involved the posterior inferior cerebellar artery(PICA). RESULTS

Placement of single stent in 5 lesions and stent-within-a-stent (diuble stent) for 8 patients. Follow-up was performed using CT angiography, digital subtraction angiography(Try to alternate follow-up by 2 methods)and MRI/MRA. Successful occlusion or gradual decreased contrast filling outside of the stent was noted in 11 patients(12 lesions), partial parent artery occlusion in 1 patient and increased aneurysmal sac in 1 case, but stabilized size at 1 year follow-up. All cases did not show remodeling of stent. No remarkable recurrent ischemic events in all cases. PICA was preserved in all those with follow-up(1 week to 4 years).

CONCLUSION

Stent placement is safe and suitalble for treatment of vertebrobasilar artery dissection by exclusion of dissected false lumen from the normal vessel, as well as prevention of vessel recoil and rupture. Futhermore, it could eliminates the possibility of more causes of thrombolembolism. But, the potential benefits of endovasucular stenting cannot be determinded, randomized control trial is needed, to compare with results of medication only.

\section{P2:38}

\section{EARLY RECANALIZATION OF ACUTE ISCHEMIC STROKE USING NEUROFORM STENTS}

S. KIM, D.H. Lee, H.K. Lim, D.C. Suh

University of Ulsan College of Medicine, Asan Medical Center, Seoul, SOUTH KOREA

\section{PURPOSE}

To report four cases with acute ischemic stroke using Neuroform stents.

\section{METHODS}

During local intra-arterial thrombolysis (LIT), management of the secondary embolization of a fragmented or dislodged clot, can be cumbersome. The fate could be dismal if one fails to pursue and lyse the migrated clot. Although to date, the occurrence and significance of clot fragmentation and distal migration during local intra-arterial thrombolysis (LIT), have not been systematically addressed, it is quite common to observe this phenomenon during the LIT. It is well-known that this phenomenon is common even when using clot retrieval devices. The distal clot migration may hamper the success of a procedure, especially when migrated clots further occlude the arteries having clinically significant perforators or cortical branches. Recently, we experienced four patients with acute ischemic stroke which were due to large clot impinged in the mid basilar trunk or distal ICA. RESULTS

We successfully managed four patients without distal migration using a self-expandable stent during LIT. The CT angiogram obtained the following day revealed complete resolution of the filling defect with good patency of the stent.

\section{CONCLUSION}

Primary stenting can be used as primary treatment modality for early recanalization in selective cases of recalcitrant occlusion. We could avoid distal clot migration by containing the clot using a self-expandable stent during local infusion of thrombolytics.

\section{P2:39}

\section{GREEK EXPERIENCE IN CADASIL}

V. KATSAROS $^{1}$, M. VIKELIS ${ }^{2}$, D.D. MITSIKOSTAS ${ }^{3}$, C. KARAGEORGIOU ${ }^{2}$, C. DROSSOS ${ }^{4}$, A.D. GOULIAMOS ${ }^{5}$

${ }^{1}$ IKA Oncology Hospital, Department of CT and MRI, Athens, GREECE, ${ }^{2}$ Athens Gemeral Hospital, Neurology Clinic, Athens, GREECE, ${ }^{3}$ Athens Naval Hospital, Neurology Clinic, Athens, GREECE, ${ }^{4}$ Athens Gemeral Hospital, Department of Modern Imaging Modalities, Athens, GREECE, ${ }^{5}$ Areaieion Hospital, Department of Radiology, University of Athens Medical School, Athens, GREECE

\section{PURPOSE}

To prospectively investigate the patterns and rates of progression of magnetic resonance (MR) imaging abnormalities in a welldocumented cerebral autosomal dominant arteriopathy with subcortical infarcts and leukoencephalopathy (CADASIL) cohort. MATERIALS AND METHODS

We describe 6 cases who were NOTCH3 mutation carriers and 15 who were non-mutation carriers who presented with a minor stroke that occurred in the absence of significant vascular risk factors. His family history included stroke, dementia and early death.

\section{RESULTS}

MRI brain scan demonstrated hyperintensities in the white matter on FLAIR images with prominent involvement of the area of the external capsule bilaterally as well as in the subcortical white matter of the temporal regions. Based on the family history and the MRI findings, CADASIL was suspected. Mutational analysis of the Notch3 gene disclosed in one patient a novel mutation substituting cysteine for glycine at codon 251 in exon 5, confirming the diagnosis of CADASIL.

\section{CONCLUSION}

CADASIL should be suspected in patients with stroke that arises in the absence of known vascular risk factors, especially if there are typical MRI findings. A strong family history of stroke and dementia are also supportive.

\section{P2:40}

\section{MULTIDETECTOR COMPUTED TOMOGRAPHY ANGIOGRAPHY (MDCTA) FOR THE EVALUATION OF CAROTID DISEASE. AN ODYSSEY BEYOND THE STENOSIS}

P. ZAMPAKIS, C. KALOGEROPOULOU, P. KRANIOTIS, E. SKONDRAS, A. KARATZAS, T. PETSAS UNIVERSITY HOSPITAL OF PATRAS.RADIOLOGY DEPARTMENT, PATRAS, GREECE 


\section{PURPOSE}

To assess the multivariate role of MDCTA for the pre-treatment evaluation of patients with carotid disease.

PATIENT-METHOD

We retrospectively analyzed 285 patients with symptomatic and asymptomatic carotid disease over the last three years.

All patients underwent a carotid and cerebral CTA, using a 16-row multi-detector CT scanner (Lightspeed 500, GE). Images were obtained from the level of the aortic arch, including the entire cranial cavity.

With the use of semi-automatic software we evaluated the carotids, in terms of the percentage of the stenosis and the pattern of atheromatous plaque.

RESULTS

Computed Tomography Angiography (CTA) accurately showed the differentiation between soft and calcified plaques, revealed ulcerated plaques, and managed to differentiate complete occlusions of the ICA from almost complete occlusion (99\%), either short or long segment (string sign).

Furthermore we managed to depict any other vascular pathology, including aneurysms, dissections, dysplastic vessels, in the entire course of extra and intra cranial carotid arteries. We also evaluated anatomic variations in the intracranial vessels.

CONCLUSION

The multidetector computed tomography of the carotids is an excellent diagnostic tool for the management of these patients and could be the sole examination for the pre-treatment planning (either surgical or endovascular).

\section{P2:41}

\section{ISCHEMIC HYPERINTENSITIES ON DIFFUSION-WEIGHTED IMAGING MAY BE REVERSIBLE AFTER THROMBOLYSIS}

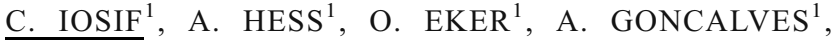
M. PASQUINI ${ }^{1}$, D. TRYSTRAM ${ }^{1}$, M. PETKOVA ${ }^{1}$, S. RODRIGO ${ }^{1}$, E. TOUZE ${ }^{2}$, C. OPPENHEIM ${ }^{1}$, J.F. MEDER ${ }^{1}$

${ }^{1}$ DEPARTMENT OF NEURORADIOLOGY, STE ANNE HOSPITAL, PARIS, FRANCE, ${ }^{2}$ DEPARTMENT OF NEUROLOGY, STE ANNE HOSPITAL, PARIS, FRANCE

\section{PURPOSE}

Diffusion-weighted Imaging (DWI) hyperintensities related to brain ischemia are usually considered irreversible. Nevertheless, these findings could resolve in case of early recanalisation. Our objective is to evaluate the frequency and factors associated with the reversibility of DWI findings after IV thrombolysis and their clinical correlates. MATERIAL AND METHODS

Among 38 consecutive stroke patients treated with IV thrombolysis in 2008, 29 patients with MRI before and after treatment $(<48 \mathrm{~h})$ were selected. The patients were divided in two categories according to the reversibility (group A) or non reversibility (group B) of the findings. Their clinical and radiological characteristics were compared with non parametric tests.

\section{RESULTS}

The DWI hyperintensities regressed partially (mean \pm SD, $64 \pm 25 \%$ volume reduction) or totally in 6 patients $(20.7 \%$ group A) and were stable or increased in 23 patients (group B). The two categories were comparable in terms of NIHSS at onset ( $13 \pm 6$ vs $13 \pm 6$; N.S.), time from onset-to-initial MRI and site of occlusion. The patients of group A were younger $(\mathrm{P}=0.02)$, thrombolysed earlier $(120 \pm 32$ vs $158 \pm 49$ minutes; $\mathrm{P}=0.06)$ and more frequently recanalyzed $(\mathrm{P}=0.04)$ than those of group B. They had a better clinical outcome 24 hours after thrombolysis (NIHSS $=2 \pm 2$ vs $11.3 \pm 7 ; \mathrm{P}=0.002$ ) and at hospital discharge (Rankin Score $1 \pm 0.9$ vs. $3.22 \pm 1.6 ; \mathrm{P}=0.006$ ). CONCLUSION

The reversibility of DWI abnormalities after IV thrombolysis was observed in about $20 \%$ of patients in our series and was associated with an excellent clinical outcome. The ischemic penumbra may include part of the DWI hyperintensities.

\section{P2:42}

\section{MULTI-DETECTOR COMPUTED TOMOGRAPHY ANGIOGRAPHY (MDCTA) FOR THE DETECTION OF ANATOMICAL VARIATIONS OF INTRACRANIAL VESSELS AND EXTRACRANIAL CAROTIDS}

\author{
P. Zampakis, S. Spiliopoulos, P. Kraniotis, A. Diamantopoulos, \\ C. Kalogeropoulou, D. Siablis
}

University Hospital of Patras, Patras, GREECE

\section{PURPOSE}

Our aim is to describe and analyze some particularly interesting anatomical variations of the intracranial arteries, and extracranial carotids detected with the use of MDCTA and to emphasize their clinical significance.

\section{MATERIAL AND METHODS}

MDCTA is routinely used in our department, for the detection of endocranial and/or extra-cranial vessel pathology with the exception of arterio-venous communications. We present 7 cases of clinically interesting anatomical variations of the head and neck vessels, detected during MDCTA imaging and post-processing data acquisition.

RESULTS

The MDCTA findings will be presented after a short but necessary introduction regarding the embryologic development of the vertebral and carotid system, which is essential for the understanding of the anatomical variations. Finally the clinical significance of these findings will be analyzed. CONCLUSIONS

The detection of the anatomical variations of the head and neck vessels using MDCTA, combines high resonance angiographic images with the visualization of the adjacent anatomical structures, which are necessary for the diagnosis of these variations. The detection and description of these entities from the radiologist provides very useful information, essential for the accurate design of any therapeutic intervention.

P2:43

\section{OPTIMIZATION OF COMPUTED TOMOGRAPHY ANGIOGRAPHY FOR THE STUDY OF CERVICAL VESSELS: THE IDEAL INJECTION- END-ACQUISITION-LAG (IDEAL) IDEA}

R. Ramos, M. Cordeiro

Hospitais da Universidade de Coimbra, Coimbra, PORTUGAL 


\section{PURPOSE}

To optimize contrast injection parameters in order to avoid the cervical veins reflux artifact (CEVRA) of intra-venous (IV) administration of iodinated contrast and stil obtain diagnostic images while minimizing contrast volume injection to decrese the risk of its side efects, namely nephrotoxicity.

METHODS

We studied 14 patients (8 male) performing CTA to control previous dissected carotids. Cranio-caudal spiral acquisition in a 64 slices GE Light Speed scanner were performed (rotation 0,5 s, detector $20 \mathrm{~mm}, 0,625 \mathrm{~mm}$ slice thicness, pitch 1, speed 19, $37 \mathrm{~mm} /$ rotation, FOV-small head, $120 \mathrm{KVolts}$, smart $\mathrm{mA}$, time acquisition $7 \mathrm{~s}$ ) after test bolus with $15 \mathrm{ml}$ of iodinated contrast (iomeprol $400 \mathrm{mg} / \mathrm{ml}$ ) at a $3,5 \mathrm{ml} / \mathrm{s}$ rate. We then injected $80 \mathrm{ml}$ of the same contrast agent beginning the acquisition at the determined time. As in most of the patients there was venous reflux that hindered part of the carotid image, we began decreasing the contrast amout in order to increase the injection-end-acquisitionlag (IDEAL), i.e., the time between the end of the contrast injection and the end of the angio-CT acquisition at the aortic arch. Then, in each new patien, the volume was successively reduced, so that the IDEAL was $2,4,6,8,10,12$ and 14 seconds. We analised the images thus obtained, looking for the CEVRA, and carotid and jugukar filling (CF and JF).

\section{RESULTS}

Patients with a 0 to $10 \mathrm{~s}$ IDEAL had CEVRA and good CF. The 3 patients with $12 \mathrm{~s}$ IDEAL had no artifacts and good CF; we injected $50 \mathrm{ml}$ of contrast in two patients and $53 \mathrm{ml}$ in the third. Patients with $14 \mathrm{~s}$ IDEAL had no CEVRA, but an sub-optimal CF. CONCLUSION

The ideal IDEAL to obtan cervical images without CEVRA and a good carotid filling is $12 \mathrm{~s}$ and in most of the times it corresponds to an administration of about $50 \mathrm{ml}$ of contrast.

\section{P2:44}

\section{POSTERIOR REVERSIBLE ENCEPHALOPATHY SYNDROME AS A COMPLICATION OF DIGITAL SUBTRACTION ANGIOGRAPHY}

\section{CORDEIRO, R. Pereira, T. Parreira, P. Freitas, C. Moura Hospitais da Universidade de Coimbra, Coimbra, PORTUGAL}

\section{PURPOSE}

To describe a previously unreported reversible complication of digital subtraction angiography (DSA).

\section{METHODS}

We report a clinical case of posterior reversible encephalopathy during and after DSA.

\section{RESULTS}

A 51 years old women presented with the worst headache of her life with no other neurologic deficits. Head computed tomography (CT) showed a perimesencephalic subarachnoid hemorrhage specially collected at the left cerebellopontine angle cistern. At the Angio-CT thus performed, no aneurysm was detected. After 36 hours she was submitted to a DSA. Femoral puncture evoked a self limited vasovagal syndrome with sudoresis, dizziness and hypotension which lasted few seconds.Vertebral artery was selected with a 5 French Simmons- 2 catheter and we manually injected $2 \mathrm{ml}$ of the non anionic iodinated contrast Ultravist 370 . After about 30 seconds the patient became non responsive, tetraplegic amaurotic and with fixed gaze on the mid line. She ventilated spontaneously, had a normal heart rate and blood pressure and had one vomit. We stopped the DSA and performed a heart CT which showed no new signs (only slight reabsorption of the sub-arachnoid haemorrhage. The patient gradually recovered from the paraplegia and aphasia about 30 minutes after the event but remained with cortical amaurosis which recovered $24 \mathrm{~h}$ after the event and $125 \mathrm{mg}$ of metilprednisolone. Brain MR showed vasogenic edema at the occipital cortex with low $\mathrm{CBV}$ and $\mathrm{CBF}$ in the perfusion study consistent with PRES.

\section{CONCLUSION}

Reversible amaurosis is an infrequent but known complication of DSA. Complete clinical and imaging features of PRES haven't, to our knowledge, been previously described. We should consider this hypothesis in a patient with suggestive clinical signs and avoid potential armful thrombolytic therapy for embolic stroke.

\section{P2:45}

\section{CEREBRAL CONTUSION OR ABSCESS?}

C. CHRISSICOPOULOS ${ }^{1}$, S. Mourgela ${ }^{1}$, K. Kirgiannis ${ }^{1}$, K. Petritsis $^{1}$, N. Ampertos ${ }^{1}$, A. Tavernaraki ${ }^{2}$, A. Spanos ${ }^{1}$

${ }^{1}$ Neurosurgical Department, Agios Savvas Anticancer Institute, Athens, GREECE, ${ }^{2}$ Department of Radiology, Agios Savvas Anticancer Institute, Athens, GREECE

\section{PURPOSE}

Head injury is one of the commonest causes for requesting cranial $\mathrm{CT}$, which can identify different pathological entities such as hematomas, contusions, abscesses, air entrapment and fractures. A ring contrast enhancement on the periphery of a parenchymal lesion can be observed in abscesses, contusions, gliomas, metastases and infarcts. We describe this case in order to show that ring enhancement around a lesion could be misinterpretated in the every day clinical practice.

Case presentation: A 38 year-old man involved in a car accident was admitted in our hospital. Brain computed tomography (CT) revealed right sided contusions in the frontal and parietal lobes. A brain CT examination performed 15 days after the injury revealed the presence of multiple hypodense areas with ring contrast enhancement in the right frontal and parietal lobes. This figure could represent either multiple contusions or abscesses. Brain MRI followed, which revealed that the lesions were brain contusions.

\section{CONCLUSIONS}

During healing process of a brain contusion there is a neovascularization phase, but the new vessels formed have defective endothelial junctures causing disturbance in blood brain barrier. Thus, at this stage, if intravenous contrast is given for cross sectional imaging, enhancement will be demonstrated, a finding that is characteristic but not specific of brain abscesses. The ring enhancement feature of a brain abscess on CT represents different degrees of encapsulation and is more common at the neovascularization phase, two weeks after the initiation of cerebritis at the beginning of the maturation process. Magnetic resonance imaging is the most reliable and more sensitive examination than $\mathrm{CT}$ in imaging cerebral abscesses. 
We conclude from this case that lesions, which show a neovascularization phase in their clinical course, can appear as ring enhancing lesions on CT study and need further investigation with MRI.

P2:46

\section{A CASE OF INTRACEREBRAL HEMATOMA DUE TO AN ANEURYSM RUPTURE WITHOUT SUBARACHNOID OR INTRAVENTICULAR HEMORRHAGE}

C. CHRISSICOPOULOS, S. Mourgela, N. Ampertos, K. Kirgiannis, K. Petritsis, A. Spanos

Neurosurgical Department, Agios Savvas Anticancer Institute, Athens, GREECE

\section{PURPOSE}

An aneurysmal rupture typically presents on computed tomographic (CT) imaging as a subarachnoid hemorrhage (SAH). A typical history of sudden severe headache, CT scan and sometimes a lumbar puncture would help clinical the diagnosis. In some rare cases aneurysmal rupture may be associated with intraparenchymal hemorrhage (IPH), intraventricular hemorrhage (IVH), or subdural hemorrhage $(<2 \%)$. We describe a rare case of aneurysmal rupture presented with IPH in order to denote the clinical significance of performing digital substraction angiography (DSA) in such a case. CASE PRESENTATION

A 52-year old woman was admitted in our hospital because of a right sided intraparenchymal hemorrhage (Fig. 1), which was localized close to the ventricular system. The clinical picture was that of low consciousness level and left paresis. From the past history no significant pathologic findings were noticed. Because of the localization of the hemorrhage we decided to perform a DSA examination, which revealed an aneurysm of the posterior cerebral artery (Fig. 2).

CONCLUSION

Initial presentation of a ruptured aneurysm without $\mathrm{SAH}$ is rare and may have a multifactorial cause attributable to the timing of CT imaging, physiological parameters, or location of the aneurysm. Patients presenting with a head CT scan revealing IPH in the temporal lobe(close to the ventricular system) or even with IVH, should be considered for an urgent workup of a ruptured aneurysm, even in the absence of diffuse SAH. In such cases, a high index of suspicion is needed to deliver the correct management.

\section{P2:47}

\section{IS COMPUTED TOMOGRAPHIC(CT) ANGIOGRAPHY A RELIABLE DIAGNOSTIC TOOL FOR THE DETECTION OF CEREBRAL ANEURYSMS?}

\section{J. CHEONG, H.J. Hong, J.M. Kim, C.H. Kim}

Hanyang University Guri Hospital, Guri, SOUTH KOREA

\section{INTRODUCTION}

Computed tomographic(CT) angiography is a promising minimally invasive method for both detection and emergency surgical planning of intracranial aneurysms. However, there are still limitations despite improving CT angiographic techniques. We describe unusual cases of false-positive aneurysms due to venous structures on preoperative CT angiography and brief overview of pitfalls of CT angiography.

CASE SUMMARY

1)A 64-year-old man presented with a 14-day history of headache associated with nausea and nuchal rigidity. On neurological examination, he showed no abnormal findings. Brain CT revealed no abnormal findings. After lumbar puncture, cerebrospinal fluid appeared xanthochromia in three successive tube. Preoperative CT angiography showing an aneurysm at the bifurcation of the left middle cerebral artery.

At operation, prominent sylvian vein superimposed on the bifurcation of the left MCA and was adhered to surrounding arteries. The hematoma existed around the vessels and there was no definite hemorrhagic focus.

2)A 45-year-old woman presented with a 2-month history of headache associated with nausea. Brain CT revealed no abnormal findings. However, the CT angiography showing an aneurysm at the proximal right middle cerebral artery. We tried conventional angiogram to rule out false-positive aneurysm and it showed no aneurysm.

CONCLUSIONS

Although CT angiography is a useful method of evaluating patients with suspected subarachnoid hemorrhage or cerebral aneurysm, close attention to image acquisition and interpretation is required to reduce a false-positive result in $\mathrm{CT}$ angiography of intracranial aneurysm.

\section{P2:48}

\section{POSTPROCESSING RECONSTRUCTIONS IN DYNAMIC CONTRAST ENHANCED MAGNETIC RESONANCE ANGIOGRAPHY OF LARGE AND GIANT CEREBRAL ANEURYSM AT PRASAT NEUROLOGICAL INSTITUTE}

\section{S. CHANYAWATTIWONGSE}

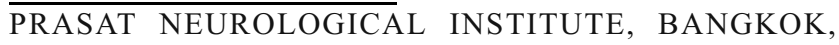
THAILAND

\section{PURPOSE}

Large $(2-2.5 \mathrm{~cm})$ and giant (larger than $2.5 \mathrm{~cm}$ ) cerebral aneurysms are difficult to identification by $3 \mathrm{D}$ time-of-flight (3D-TOF) MR Angiography (MRA) due to signal loss by spin saturation and intravoxel phase dispersion. Dynamic contrast enhanced MR angiography was well demonstrated these aneurysms. The aim of this study was to study postprocessing reconstructions modalities maximum intensity projection (MIP) and volume rendering technique (VRT) to evaluate large and giant cerebral aneurysm in dynamic contrast enhanced MR angiography at Prasat Neurological Institute.

METHODS

3 Patients (1 male, 2 females, 1 large aneurysm, 2 giant aneurysm) had undergone 3D-TOF and 3D gradient recalled echo dynamic contrast enhanced MR angiography with 1.5 tesla MR system between January 2008 and February 2009. For postprocessing reconstructions, maximum intensity projection 
and volume rendering technique were used. The results were evaluated by the well detection of aneurysm neck, dome morphology and adjacent vessels.

RESULTS

In this study 3D-TOF is inadequate to show large and giant cerebral aneurysms. Dynamic contrast enhanced MR angiography allows the visualization of aneurysm and the temporal resolution provides a separation of arteries and veins. Postprocessing reconstructions, VRT is superior to MIP as it provides better morphology of aneurysm sac and connected vessels for the detection of large and giant cerebral aneurysms.

\section{P2:49}

\section{CRANIAL MAGNETIC RESONANCE VENOGRAPHY: NORMAL ANATOMY AND VARIATIONS}

M. APAYDIN, E. DOGAN, M. APAYDIN, M. VARER, A. SARSILMAZ, O. OYAR, E. ULUC

IAEAH- Department of Radiolgy, IZMIR, TURKEY

The aim of this study was to evaluate the variations of cerebral venous and dural sinuses anatomy and signal defects by using $2 \mathrm{D}$ time-of-flight(TOF) cranial magnetic resonance(CRMRV) technique. Seventy-eight patients with clinical findings suggestive of cranial venous and dural sinus thrombosis with normal CRMRV, were examined retrospectively. They were rewieved to determine the presence or absence of the dural sinuses, major intracranial veins and signal defects especially in the transvers sinuses.

The review of the 78 cases revealed that, all the cases had internal cerebral and galen veins. The basal vein of Rosenthal were seen $97.5 \%$. The superior sagittal,transvers, sigmoid and straight sinuses were seen in every venogram; the inferior sagittal sinus was seen in $94 \%$. There was hypoplasia of the right sigmoid sinus in $35 \%$ and left sigmoid sinus $43 \%$. The juguler veins were revealed the same characteristics.But in the transverse sinuses, the $14-18 \%$ were showed codominancy. Flow gaps were observed in non-dominant transverse sinus, sigmoid sinus as well as transverse sigmoid sinus junctions. Transverse sinus flow gaps in $37 \%$ of the cases, with $75 \%$ of these occurring in the nondominant transverse sinus and $25 \%$ in the codominant transverse sinuses. The occipital sinus was visualized in $6 \%$.

CRMRV deliniates major cerebral venous and dural sinuses. Awareness of the normal anatomical variations of venous sinuses and CRMRV signal defects prevent misdiagnosis of cerebral venous and dural sinus thrombosis.

\section{P2:50}

\section{ENDOVASCULAR EMBOLIZATION OF PARTIALLY CLIPPED ANEURYSMS}

E. AKGUL, T. BALLI, S. ILHAN, F. BINOKAY, E. AKSUNGUR CUKUROVA UNIVERSITY, FACULTY OF MEDICINE, RADIOLOGY DEPARTMENT, ADANA, TURKEY

\section{PURPOSE}

We aimed the endovascular embolization of intracranial aneurysms partially clipped surgically.

\section{MATERIALS AND METHODS}

3 patients with intracranial aneurysms which could not be clipped totally were included in the study. They were 2 males and 1 female with ages ranging between 29 and 53 years. All patients were operated on because of ruptured aneurysms and partially clipped aneurysms were detected on angiographic control. Patient with anterior communicating aneurysm was embolized 10 days after operation. Patients with right posterior communicating and right media aneurysms were embolized 3 months after operation. In all patients, neck remodelling balloons (Hyperform $4 \times 7 \mathrm{~mm}$ and Hyperglide $4 \times 20 \mathrm{~mm}$, Micro Therapeutics, USA) were used. Posterior cerebral artery aneurysm in patient with media aneurysm was also embolized in same session. In addition, a stent (Leo stent, Bard) was used in patient with posterior communicating aneurysm, due to wide neck of aneurysm. 1 patient was controlled angiographically after 3 months.

RESULTS

All aneurysms but posterior communicating aneurysm were totally embolized.

There was a minimal neck filling in the posterior communicating aneurysm. There was no progression in this residual filling. Angiographic control could not be performed on the other 2 patients because they did ot show up for appointment. No addditional neurologic deterioration was seen.

\section{CONCLUSION}

Endovascular embolization is an alternative, safe and effective method in the treatment of residual aneurysm after surgical clipping.

\section{P2:51}

\section{CASE REPORT: UNILATERAL MOYAMOYA DISEASE}

\section{G. LUCENTE $^{1}$, M. RAGUSO ${ }^{2}$, I. FRANCAVILLA ${ }^{1}$}

${ }^{1}$ Ospedale A. Perrino - U.O. Radiologia Interventistica, Brindisi, ITALY, ${ }^{2}$ Ospedale S. Marco - Reparto di Radiodiagnostica, Grottaglie, ITALY

\section{PURPOSE}

Case report of a young man, nineteen years old, comes in our hospital with recent anamnesis of headache and seizure and signs of acute stroke.

\section{METHODS}

Diffusion magnetic resonance (DWI-MRI), reveal a recent stroke in globus pallidus, putamen and internal capsule. Angio-MRI and Computed Tomography (CT) and Angio-CT shows generic vasculopathy in the clinoid part of left internal carotid artery (ICA) and in tract M1 of left media carotid artery (MCA). Angiography shows stenosis of clinoid left ICA and steno-occlusion of tract M1 of left MCA with enlarged lenticulo-striate and thalamoperforating arteries who gives the tipical "puff of smoke" appearance of Moyamoya desease (MD); aplasia of A1 of left anterior cerebral artery (ACA). Collateral leptomeningeal and dural from branches of ECA support the circle of distal tract of MCA and, between comunicant anterior artery, the Circle of Willis fill left ACA.

\section{RESULTS}

$\mathrm{MD}$ is a angiography diagnosis of progressive steno-occlusive process, that tipically involve bilateral clinoid portion of ICA and/or the proximal portion of ACA and MCA in children or young adult; unilateral MD is recognised in $18 \%$ of the cases. The management of 
patient with this chronic and progressive vasculopathy have two options, in early stage often is choosen the "wait and see" behaviour with rigorous follow-up in the others cases is preferred the surgical by-pass. In our case, after an exam of Single Photon Emission Computed Tomography (SPECT) who shows non significant deficit of perfusion of the left side of the brain, we decided to plane a followup (MRI/Angio-MRI and Angiography) who is still going on.

CONCLUSIONS

Unilateral MD is finded in a small rate of a rare chronic and progressive vasculopathy; angiography gives diagnosis and imaging of cerebral perfusion helps us to manage the patient.

\section{P2:52}

\section{DIAGNOSTIC CRITERIAS OF HYPOGLOSSAL DURAL ARTERIOVENOUS FISTULA: ABOUT SEVEN CASES}

A.L. Derelle $^{1}$, R. Anxionnat ${ }^{1}$, A. Lebedinsky ${ }^{1}$, A. Aly ${ }^{1}$,

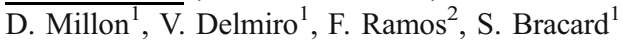

${ }^{1}$ Service de Neuroradiologie CHU Nancy, Nancy, FRANCE, ${ }^{2}$ Servico de Neurorradiologia HC / incor FMUSP, Sao Paulo, BRAZIL

\section{PURPOSE}

The hypoglossal canal is an uncommon topography of skull base dural arteriovenous fistula (AVF). Diagnostic is important to assess because this location is usually and easily treated by venous way.

\section{PATIENTS AND METHODS}

We retrospectively rewieved 13 posterior fossa fistulas. Among these, an hypoglossal location was diagnosed in 7 patients. CTangio and MRA were performed in 2 patients. All patients underwent angiography, including 3D angiography in one patient. RESULTS

AngioCT revealed asymmetrical enhancement in one case and enlargement of hypoglossal canal in another case. MRA in 3dTOF demonstrated high flow in hypoglossal canal in one case highly suggestive of hypoglossal fistula.

Diagnosis was definitely assessed by angiography for the seven patients.

Arterial afferences in all the cases of hypoglossal fistula were bilateral ascending pharyngeal and ipsilateral internal carotid arteries. Compared to the other posterior fossa fistulas, afferences by the controlateral ascending pharyngeal artery is the discriminating point. Drainage through the anterior condylar vein was constant for hypoglossal fistulas but was seen in 2 others posterior fossa fistulas.

The shunting point was best demonstrated on the contralateral pharyngeal artery angiogram. 3D angiography performed in one case allowed to localize precisely the fistula inside the hypoglossal osseous canal.

CONCLUSION

Hypoglossal dural AVF could be strongly suggested by angioCT and MRA using 3D TOF. The diagnosis is definitely assessed on selective angiography : afference from contralateral ascending pharyngeal artery was the discriminating point for the diagnosis. 3D unsubstracted MIP reconstructions demonstrate the foraminal location of the shunt and the precise architecture of the dural AVF.
P2:53

\section{THE ROLE OF CT-VENOGRAPHY IMAGING ON THE DIAGNOSIS OF AN ISOLATED CORTICAL VEIN THROMBOSIS CASE}

J. MARTINS, G. SANTOS, C. CASIMIRO, D. MARNOTO

University Hospital of Coimbra - Division of Neuroradiology University Clinic of Imaging, Coimbra, PORTUGAL

\section{PURPOSE}

Isolated cortical vein thrombosis (ICVT) is a rare subtype of cerebral vein thrombosis. Its diagnostic is challenging, due to anatomic variability of cortical veins, and complicated by a wide range of unspecific clinical presentation. Diagnosis is usually based on magnetic resonance imaging (MRI) with Angiographic study or on Digital subtraction angiography. The role of cerebral CT-Venography is less well established in the literature.

The authors present a case of ICVT diagnosed by cerebral CTVenography, compare the findings of this exam with those of MRI, and discuss the role of cerebral CT-Venography imaging on initial diagnosis of ICVT as an alternative to MRI.

METHODS AND RESULTS

A 39-year-old female presented to emergency room with a onemonth complaints of headache and superior limbs and left inferior limb paresthesia. On exam, left superior limb hipostesia was noted. Initial head CT was normal; a week later, patient again underwent head CT scan that showed an area of hyperdensity in left posterior frontal high convexity, involved by hipodensity of the surrounding brain, suggestive of venous infarction. Cerebral CT-Venography with $3 \mathrm{D}$ reconstructions showed, on the same location, lack of filling of the proximal portion of a cortical vein; cerebral MRI, on T1-weighted images, showed spontaneous hyperintensity of a cortical vein and $\mathrm{T} 2 *$-weighted gradient-echo (T2*GE) images identified a tubular, serpentine hypointensity with blooming. The patient symptoms improved and, a week later, CT-Venography showed repermeabilization of the cortical vein, with residual hipodensity of the surrounding brain. The patient is nearly asymptomatic.

\section{CONCLUSIONS}

Our case seems to suggest that CT-Venography can be a good alternative to MRI for the diagnosis of ICVT. The 3D reconstructions were especially useful to establish the diagnosis, by simplifying the visualization of the cortical veins anatomy. Follow-up is better evaluated with MRI, especially $\mathrm{T} 2 * \mathrm{GE}$ sequence, because of its high sensitivity to the paramagnetic products of hemoglobin.

\section{P2:54}

\section{ACCURACY OF MRA (MAGNETIC RESONANCE ANGIOGRAPHY) FOR THE DETECTION OF INTRACRANIAL ANEURYSM AS COMPARED TO DSA (DIGITAL SUBSTRACTION ANGIOGRAM)}

\author{
P. METARUGCHEEP, N. KIATHRIRANON, \\ S. CHANYAWATTIWONGSE \\ PRASAT NEUROLOGICAL INSTITUTE, BANGKOK, \\ THAILAND
}




\section{PURPOSE}

To assess the role of MRA for detection of intracranial aneurysm as compared to DSA by using strandard method of post processing (MIP)in the blinded reader study.

\section{METHOD}

Two hundred vessels were examined with DSA and 3DTOFMRA in 50 patients with SAH ( subarachnoid hemorrhage) :38 ameurysms,1 DAVF (dural arterio-venous fistula) and 13 patients with no aneurysm at DSA. MRA were interpreted by neuroradiologist blinded to the DSA results for presence, location, size and morphology of the aneurysm.

\section{RESULTS}

Mean sensitivity for the detection of aneurysm was $92.1 \%$, specificity $100 \%$, positive predictive value $100 \%$ and negative predictive value $81.3 \%$. 38 aneurysms were found : A-com 14, Pcom 8 , MCA 7 , BCA 4 , ICA 3 , VBA 1 , DAVF 1 and 13 no aneurysm. True positive 35 , true negative 13 , false positive 0 and fasle negative 3 .

CONCLUSION

MRA with standard post processing can result in high sensitivity and specificity for the diagnosis of intracranial aneurysm, sufficient size to be considered for surgical treatment. The positive predictive value is also very high but the negative predictive value is not high enough. Therefore, in the negative study of MRA,DSA is still recommended.

\section{Free Topics}

\section{P3:55}

\section{IDENTIFICATION OF DILATED VIRCHOW-ROBIN SPACES ON MR IMAGING}

E. Testempasi ${ }^{1}$, K. Stefanidis ${ }^{1}$, V. Ouranos $^{1}$, S. Benakis ${ }^{1}$, C. Louizakis ${ }^{2}$, I. Skouras ${ }^{1}$, E. Kratimenou ${ }^{1}$, A. Sykara ${ }^{1}$, D. Chondros ${ }^{1}$

${ }^{1}$ Evangelismos Hospital, CT \& MRI Department, Athens, GREECE, ${ }^{2}$ Policliniki Hospital, Radiological Department, Athens, GREECE

\section{PURPOSE}

VR spaces are pial lined extensions of the subarachnoid space that surround small arteries and arterioles as they perforate the surface of the brain and extend into the brain tissue. The objective of this study is to describe and illustrate the MR radiological features of perivascular spaces also known as Virchow-Robin (VR) spaces in order to recognize and differentiate these normal anatomic structures from pathologic lesions.

\section{METHODS}

In this study a total of 443 consecutive patients, who underwent brain MR examination, were retrospectively evaluated to investigate the presence of dilated VR spaces (191 men, 252 women, Mean age: 61 y.o., range $20-86$ y.o.). An MR scan was performed to rule out any structural lesion in the brain.

\section{RESULTS}

Dilated VR spaces were visible in 23 patients. On MR imaging, dilated VR spaces appeared round, oval or curvilinear with a well defined, smooth margin and with no mass effect. They did not enhance with contrast material and they appeared isointense to cerebrospinal fluid (CSF) in all sequencies. Their localization was along the lenticolostriate arteries entering the basal ganglia through the anterior perforated substance, along the path of the perforating medullary arteries and in the midbrain. The differential diagnosis includes other lesions isointense to CSF such as lacunar infarctions, cysts (ependymal cyst, neuroepithelial cyst and arachnoid cyst), cystic periventricular leucomalacia, multiple sclerosis, cystic neoplasms and mucopolysaccharridosis.

\section{CONCLUSION}

Virchow-Robin spaces are a common finding that can be seen on MR images in all ageâ $€^{\mathrm{TM}_{\mathrm{S}}}$ group. Knowledge of their signal intensity characteristics and localization is of great importance for the radiologist in order to recognize the radiological features of these normal anatomic structures and differentiate them from pathologic conditions.

\section{P3:56}

\section{RADIOTHERAPY OF PITUITARY ADENOMAS}

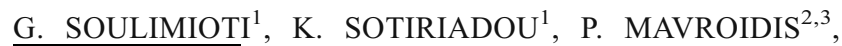
G. KYRGIAS ${ }^{1}$

${ }^{1}$ Department of Radiotherapy-Larissa University Hospital, Larissa, GREECE, ${ }^{2}$ Department of Medical Physics-Larissa University Hospital, Larissa, GREECE, ${ }^{3}$ Department of Medical Radiation Physics-Karolinska Institutet and Stockholm University, Stockholm, SWEDEN

\section{PURPOSE}

Pituitary adenomas are tumors that occur in the pituitary gland, and account for about $10 \%$ of intracranial neoplasms. The aim of this work is to determinate the role of radiotherapy in the management of pituitary $\phi s$ adenomas.

\section{MATERIAL}

The radiotherapy technique for the adenomas of pituitary is the 3Dconformal. MRI and CT for planning helps to define the treatment volume (pituitary fosse and adjacent tissues) with a margin of not more than $3-5 \mathrm{~mm}$. The patients head must be fixed with a thermoplastic immobilization mask. This procedure guaranties precise repositioning over the course of treatment. The CT for planning was acquired with the patient in the treatment position and the data was transferred in a planning system in which the radiation oncologist will define the target volume and then the medical physicist develops the treatment plan of radiotherapy with the best coverage of the target volume and the least radiation to normal and sensitive tissues (chiasma, optic nerve etc). For pituitary adenomas the best plan is a three portal arrangement with an open vertex field and two lateral beams with wedge. The daily dose is $180 \mathrm{cGy}$ and the total dose 5000-5400 cGy with 6MV photons. RESULTS

As an alternative option to medical and surgical managements, the radiation therapy is used in the treatment of pituitary adenomas because:

1. Percontrol of hypersecretion

2. Control of mass effects (decompression of optic chiasma)

3. Subtotal resection

4. Inoperable tumors

5. Recurrence after surgey

CONCLUSIONS

Radiation therapy is very effective for pituitary adenomas because it controls the hypersection ( $80 \%$ for acromegalia, $50 \%$ for Cusching, $50 \%$ for prolatinemia) diminish the mass effects (decompression of 
optic chiasma) and diminish the recurrence (postoperative radiation offers subtotal resection).

\section{P3:57}

\section{INCIDENCE OF PITUITARY INCIDENTALOMAS IN PATIENTS WITH ADRENAL ADENOMAS}

C. SAMARA ${ }^{1}$, G. KALTSAS ${ }^{2}$, T. SOLDATOS ${ }^{1}$,

I. ANDROULAKIS ${ }^{2}$, A. CHRYSALIDOY ${ }^{2}$,

Z. NIKOLAKOPOULOU ${ }^{1}$, C. DROSSOS ${ }^{1}$

${ }^{1}$ Department of Radiology and Imaging, G.Gennimatas General State Hospital, Athens, GREECE, ${ }^{2}$ Department of Endocrinology, G.Gennimatas General State Hospital, Athens, GREECE

\section{PURPOSE}

Adrenal and pituitary incidentalomas are commonly discovered by cross sectional imaging. As the frequency of pituitary incidentalomas has not been estimated before, we investigated the incidence of the latter in patients with adrenal adenomas.

METHODS

From the 24 patients (17 females, $61 \pm 12$ years old) who were prospectively evaluated, 16 (group A) had known adrenal adenoma(s), while 8 (group B) had normal adrenal morphology. All subjects underwent magnetic resonance imaging of the pituitary gland, which included conventional and contrastenhanced T1W images, as well as T2 images in the coronal and sagittal planes.

\section{RESULTS}

From the patients of group A, one was found to have a $4 \times 5 \mathrm{~mm}$ pituitary cyst and one revealed an empty sella. There was no evidence of a pituitary incidentaloma in any patient of both groups.

\section{CONCLUSIONS}

Despite the limited sample size of the study, no association was exhibited between adrenal adenomas and pituitary incidentalomas. However, further studies in larger populations are required to establish the above result.

\section{P3:58}

\section{PRESEPTAL PROTRUSION OF RETROORBITAL FAT CLINICALLY MIMICKING LACRIMAL GLAND ENLARGEMENT IN PATIENTS WITH THYROID-ASSOCIATED ORBITOPATHY}

C. SAMARA ${ }^{1}$, K. CHATZISTEFANOU ${ }^{2}$, T. SOLDATOS $^{1}$, Z. NIKOLAKOPOULOU ${ }^{1}$, E. KROMMIDA ${ }^{1}$, C. DROSSOS $^{1}$

${ }^{1}$ Department of Radiology and Imaging, G.Gennimatas General State Hospital, Athens, GREECE, ${ }^{2}$ Department of Opthalmology, G.Gennimatas General State Hospital, Athens, GREECE

\section{PURPOSE}

Thyroid-associated orbitopathy (TAO) is an autoimmune inflammatory process that affects the orbital tissues, mainly causing enlargement of the extraocular muscles and retroorbital fat. However, protrusion of the latter in the preseptal space and concomitant lacrimal gland involvement has rarely been reported in TAO. We describe 3 cases with TAO and unusual clinical and magnetic resonance imaging (MRI) findings.

\section{METHODS}

Three patients (2 males, age range 29-56 years) with clinically and biochemically established Grave's disease underwent MRI of the orbits for the evaluation of bilateral soft-tissue masses which protruded under the upper eyelids mimicking lacrimal glands enlargement. Conventional, contrast-enhanced, fat-suppressed T1 images as well as T2 images were acquired in axial, coronal and sagittal planes.

RESULTS

All MRI studies revealed bilateral enlargement of all or part of the extraocular muscles with sparing of the respective tendinous insertions, as well as bilateral protrusion of the retroorbital fat in the preseptal space. In two patients bilaterally and in one patient unilaterally the lacrimal gland was small in size and was displaced laterally by the protruding fat. In the latter subject the contralateral gland was not identified in its anatomic location. CONCLUSIONS

In TAO the retroorbital fat may expand to the preseptal space and mimic enlargement of the lacrimal gland. In such cases the gland may undergo atrophy and lateral displacement, possibly due to inflammatory infiltration and retroorbital fat protrusion. Hence, the interpretation of orbital MRI studies in patients with TAO should include the careful evaluation of the retroorbital fat and lacrimal glands in order to establish possible pathology and explain potential clinical findings.

P3:59

\section{MAGNETIC RESONANCE IMAGING FINDINGS OF CARBONMONOXIDE INTOXICATION CASES}

\author{
M. VARER $^{1}$, M. APAYDIN ${ }^{1}$, A. SARSILMAZ ${ }^{1}$, \\ Y. OZTURK $^{1}$, S. DERICI ${ }^{2}$, O. OYAR ${ }^{1}$ \\ ${ }^{1}$ ATATURK STATE AND EDUCATION HOSPITAL, RADIOL- \\ OGY, IZMIR, TURKEY, ${ }^{2}$ ATATURK STATE AND EDUCA- \\ TION HOSPITAL, NEUROLOGY, IZMIR, TURKEY
}

\section{INTRODUCTION}

Carbonmonoxide $(\mathrm{CO})$ is a colourless, odourless, tasteless and nonirritating toxic gas which can cause neurological mortality and morbidity. Clinical presentation of $\mathrm{CO}$ poisoning depends on the duration and intensity of the exposure. Magnetic Resonance Imaging (MRI) findings depend on the severity of intoxication. We report three CO intoxication cases.

\section{CASE REPORT}

Patient one was a 64 years old woman. She was brought to emergency room because of muteness, immobility and loss of social relation. Patient two was a 46 years old man who had unconsciousness and respiratuary distress. They both had increased signal intensity on both globus pallidus on $\mathrm{T} 2 \mathrm{~W}$ MR images. Patient three was a 48 years old woman who fainted at home. She had the same MRI findings, in addition she had small hiperintense changes on the left substantia nigra and on the white matter areas.

DISCUSSION

Possible mechanism of CO toxicity includes decrease in oxygen carrying capacity of blood, alteration in dissociation characteristic of oxyHb further decreasing oxygen delivery to tissues, decrease in cellular respiration by binding with cytochromes, binding to myoglobin causing myocardial and skeletal muscle dysfunction 
resulting in impaired tissue perfusion. The most common site of involvement is bilateral globus pallidus.Also abnormalities can be found in other basal ganglia structures, cerebral white matter, hippocampuscerebral cortex and cerebellum. The most common involvement of globus pallidus may be due to hypotensive effect of $\mathrm{CO}$ poisoning in the watershed territory of the arterial supply. CONCLUSION

MRI is superior to CT in diagnosing the hypoxic changes. Also MRI is useful to determine overdose and prognosis.

\section{P3:60}

\section{WHAT CAN CT AND MR SAY ABOUT THE OUTCOME OF PATIENTS WITH DIFFUSE AXONAL INJURY?}

J. SANCHEZ ${ }^{1}$, P. PUYALTO ${ }^{2}$, P. CUADRAS ${ }^{2}$, S. CASTAÑER ${ }^{1}$, S. MOURELO $^{2}$, I. URRA ${ }^{2}$, C. AGUILERA ${ }^{1}$, A. OLAZABAL ${ }^{2}$

${ }^{1}$ IDI-Hospital Bellvitge-Duran i Reynalds-Neuroradiology Department, Barcelona, SPAIN, ${ }^{2}$ Hospital Germans Trias i Pujol, Barcelona, SPAIN

\section{PURPOSE}

1) To illustrate $\mathrm{CT}$ and MR imaging findings of diffuse axonal injury (DAI)

2) To discuss the role of CT and MR imaging signs that allow a prompt diagnosis and outcome prediction of DAI.

3) The importance of advanced MR techniques such as DTI will also be discused.

\section{METHODS}

Imaging features of 36 patients ( $73 \%$ men and $27 \%$ women), aged 17-63, with mild-severe head trauma diagnosed of DAI (clinically, CT and MRI) were retrospectively reviewed.

All subjects had at least an initial CT at the moment of the trauma, a 24 hours CT and ,5T MR performed within 2 to 6 weeks after trauma. DAI lesions were classified as haemorrhagic or non hemorrhagic and by its location within the cerebral hemisphere white matter interface (Type I),corpus callosum(Type III) and dorsolateral brainstem(Type III).

\section{RESULTS}

$55,6 \%$ of our patients showed no evidence of DAI in initial CT,38,8\% evidenced intraventricular haemorrhage and 5,6\% showed puntacte haemorrhagic foci. MR demonstrated corpus callosum haemorrhagic lesions in $85,7 \%$ of the patients that showed intraventricular haemorrhage in initial CT.

In the subacute phase,T2 weighted images and mostly FLAIR weighted images were more sensitive than T1 weighted images in detecting non haemorrhagic lesions; whereas T2 gradient- echo weighted images were more sensitive in detecting haemorrhagic lesions.

\section{CONCLUSION}

In our institution, CT is the initial modality of choice for the acutely injured patient; but once the patient is stabilized, MR is performed due to its superior sensitivity for the detection of DAI in its most common locations. Based on the information of the trauma mechanism, location, number and size of the non hemorrhagic or hemorrhagic lesions within the brain assessed by CT and mainly by an appropriate combination of sequences of MRI, an accurate diagnosis and prognosis prediction of DAI can be made.
P3:61

\section{UNILATERAL HYPOPLASIA OF THE ROSTRAL SUPERIOR SAGITTAL SINUS}

D. SAN MILLAN RUIZ ${ }^{1}$, J. FASEL ${ }^{2}$, P. GAILLOUD ${ }^{3}$

${ }^{1}$ Service of Diagnostic and Interventional Neuroradiology, Department of Radiology, Geneva University Hospital, Geneva, SWITZERLAND, ${ }^{2}$ Anatomy Section, Department of Cellular Biology and Physiology, Geneva University, Geneva, SWITZERLAND, ${ }^{3}$ Division of Interventional Neuroradiology, The Johns Hopkins Hospital, Baltimore, MD, USA

\section{PURPOSE}

To report the existence of unilateral hypoplasia of the rostral superior sagittal sinus.

\section{METHODS}

100 whole-head dynamic subtracted angiography studies using a 320-multidetector row CT (WHDSCTA) in 100 consecutive pediatric and adult patients were retrospectively reviewed for the presence of unilateral HSSS. The presence of bilateral HSSS were also noted. Associated dural venous sinus anomalies were noted in all patients. The angiographic anatomy of the unilateral HSSS was illustrated by two cases investigated with DSA.

\section{RESULTS}

Unilateral HSSS was found in 7\% of the patients, of which three had other variations of the dural venous sinuses. In all cases, compensatory drainage occurred through a large superior frontal vein that joined the SSS in the region of the coronal suture. 3 out of the 7 patients with a unilateral HSSS had at least another dural venous sinus anomaly. $3 \%$ of the patients had a complete or bilateral HSSS. CONCLUSION

Unilateral HSSS is more than twice as frequent as bilateral HSSS. It is the most frequently encountered variation of the SSS. Knowledge of this anatomical variation is important to avoid diagnostic pitfalls and erroneously mistaking it for a thrombosis. Four types of variations of the rostral SSS may be identified: 1) 'classic anatomy' with a fully developed rostral SSS; 2) duplication of the rostral SSS; 3) complete or bilateral HSSS; 4) unilateral HSSS. Emergence of the four types of rostral SSS variations may be explained by studying the embryological development of the SSS.

\section{P3:62}

\section{INTRACRANIAL NEURENTERIC CYSTS - RARE INCIDENTAL FINDINGS WITH TYPICAL MR SIGNAL CHARACTERISTICS}

\section{S. PROTHMANN ${ }^{1}$, R. Trampel ${ }^{2}$, D. Fritzsch $^{3}$, J.P. Schneider ${ }^{4}$ ${ }^{1}$ University hospital rechts der Isar, Department of Neuroradiology, Munich, GERMANY, ${ }^{2}$ Max Planck Institute for Human Cognitive and Brain Sciences, Department of Neuro- physics, Leipzig, GERMANY, ${ }^{3}$ Leipzig university hospital, De- partment of Neuroradiology, Leipzig, GERMANY, ${ }^{4}$ Radiology group practice am Diakonissenhaus, Leipzig, GERMANY}

Intracranial neurenteric cysts are extremely rare congenital spaceoccupying extra-axial lesions, usually located near the midline in the posterior fossa. They can cause a variety of neurological symptoms; 
however, they occasionally appear as incidental findings in MRI. They mostly display typical MR signal characteristics: Hyperintense to cerebrospinal fluid (CSF) in T1-, T2-weighted imaging and in the Fluid Attenuated Inversion Recovery (FLAIR) sequence, while showing no restriction in diffusion weighted imaging and no enhancement. Herein, we present three cases from our clinic.

\section{P3:63}

\section{HYPERINTENSE BASAL GANGLIA ON T1 WEIGHTED MRI IN RENDU-OSLER-WEBER DISEASE}

A. OIKONOMOU ${ }^{1}$, P. MINTZOPOULOU ${ }^{1}$, A. CHATZISTEFANOU ${ }^{1}$, K. VADIKOLIAS $^{2}$, J. HELIOPOULOS ${ }^{2}$, P. PRASSOPOULOS ${ }^{1}$

${ }^{1}$ UNIVERSITY HOSPITAL OF ALEXANDROUPOLIS, Department of Radiology, ALEXANDROUPOLIS, GREECE, ${ }^{2}$ UNIVERSITY HOSPITAL OF ALEXANDROUPOLIS, Department of Neurology, ALEXANDROUPOLIS, GREECE

\section{PURPOSE}

To present changes in basal ganglia in Rendu-Osler-Weber disease on MRI.

\section{MATERIALS AND METHODS}

A 60 year old man with history of Rendu-Osler- Weber disease and episodes of epistaxis, multiple telangiectases of the lips, tongue and fingertips, presented with an episode of gastric bleeding. During the investigation of CNS involvement an MRI of the brain was performed. RESULTS

Cranial MRI depicted significantly increased signal intensity in the basal ganglia and specifically in the globus pallidus bilaterally, as compared to white matter on T1-WI images. Cerebral crura exhibited also high signal intensity on T1-WI images. There was no enhancement after gadolinium administration. T2 and FALIR sequences depicted normal basal ganglia regarding both size and signal intensity. This finding was consistent with deposition of paramagnetic substance, most probably manganese. Laboratory investigation verified manganese accumulation in the peripheral blood: manganese 5,2 ìg/dl (normal 0,8-2,5 ig/dl).

CONCLUSION

Rendu-Osler- Weber disease may cause alterations of the signal intensity on T1-WI MRI at the basal ganglia, as well as at the cerebral crura. The latter finding has not been previously described. This increase in signal intensity is attributed to deposition of manganese that may cause extrapyramidal syndromes and parkinsonism. Cranial MRI may be valuable in detecting manganese toxicity in RenduOsler-Weber disease even at initial stage of the disease.

\section{P3:64}

\section{BRAIN ABNORMALITIES IN ASPHYXIA}

A. OIKONOMOU ${ }^{1}$, P. ARGYROPOULOU ${ }^{1}$, G. KARAGIANNAKIS ${ }^{1}$, E. ASTRINAKIS $^{1}$, J. PNEVMATIKOS ${ }^{2}$, P. PRASSOPOULOS ${ }^{1}$

${ }^{1}$ UNIVERSITY HOSPITAL OF ALEXANDROUPOLIS, Department of Radiology, ALEXANDROUPOLIS, GREECE, ${ }^{2}$ UNIVERSITY HOSPITAL OF ALEXANDROUPOLIS, Intensive Care Unit, ALEXANDROUPOLIS, GREECE

\section{PURPOSE}

To describe the CT and MRI changes of brain parenchyma in two cases of prolonged asphyxia. MATERIALS AND METHODS

A 16-year old girl with history of recurrent crises of asphyxia due to myasthenia gravis for four months, and a 3 year old boy with a prolonged near-drowning episode while swimming are presented. Both patients were admitted in the hospital cyanotic and intubation was performed due to acute airway obstruction and respiratory failure. CT and MRI examinations were performed. RESULTS

The first patient had an MRI elsewhere four months prior to admition that was unremarkable. MRI in our hospital showed areas of high signal intensity in T2 and FLAIR sequences in the head of the caudate lobe and in the putamen bilaterally with no gadolinium enhancement. T1-WI images were unremarkable. One month follow-up MRI revealed progression of disease with high signal intensity in both T1 and T2 -WI images in the basal ganglia, as well as in subcortical areas of the parietal and occipital lobes exhibiting peripheral gadolinium enhancement. In the second case CT disclosed diffusely and symmetrically hypodense lentiform nuclei and thalami and a small well-defined hypodense lesion in the left globus pallidus.

CONCLUSION

Brain changes in asphyxia may present with hypodensity of basal ganglia on CT. On MRI the may present with high T1, T2 and FLAIR signal intensity of basal ganglia and subcortical regions with enhancement.

\section{P3:65}

\section{THE CLINICAL IMPACT OF 1.5 T NEUROLOGIC MRI IN PATIENTS WITH A PERMANENTLY IMPLANTED PACEMAKER: A NECESSARY PROCEDURE FOR INDICATED CASES}

\author{
G. MILLER $^{1}$, N. CAMPEAU ${ }^{1}$, W. SHEN $^{2}$, J. FELMLEE ${ }^{1}$, \\ R. WATSON 1 \\ ${ }^{1}$ MAYO CLINIC DEPARTMENT OF RADIOLOGY, ROCHESTER, \\ NY, USA, ${ }^{2}$ MAYO CLINIC DEPARTMENT OF CARDIOLOGY, \\ ROCHESTER, NY, USA
}

\section{PURPOSE}

Current guidelines state that an implanted pacemaker is a relative contraindication for MRI. Investigators have reported that MRI can be performed safely if certain criteria are met and precautions taken. The purpose of our study was to determine the clinical impact of 1.5T MRI in pacemaker patients at our institution.

METHODS

A recently developed pacemaker protocol was strictly followed. The indication for the MRI was reviewed by neuroradiology. Imaging alternatives were considered and discussed with ordering physicians. Patients were confirmed to be non-pacemaker dependent, and pacemaker parameters were interrogated prior to and after the MRI by cardiology. Neuroradiology, cardiology and medical physics staff were present during scanning to tailor the exam to the clinical question, monitor the effect of the magnetic field and gradients on the cardiac rhythm, and modify the imaging parameters to maintain SAR below 1.5 watts $/ \mathrm{kg}$. Patient histories, 
prior imaging examinations and MRI studies were reviewed to determine the effect the MRI study had on patient management.

\section{RESULTS}

Thirty four 1.5 Tesla MRI examinations of the head and/or spine were performed on 22 patients for a variety of indications. All exams were completed without incident. A variety of pathologic conditions were identified. For 17 of 23 patients, the MRI study was determined to be critical to patient management, resulting in surgical intervention in 9 of the 22 patients $(41 \%)$ and significantly altered treatment in another $8(36 \%)$. In 5 patients, the MRI added value to patient management.

\section{CONCLUSIONS}

MRI examinations at 1.5 Tesla in pacemaker patients have the ability to significantly affect patient care and offers diagnostic information not available by other studies. This study illustrates the need for MR imaging in patients with implanted pacemakers. Further work regarding the safety and efficacy of performing MRI in pacemaker implanted patients is warranted.

\section{P3:66}

\section{PITUITARY ADENOMAS MRI-CORRELATION WITH HISTOLOGY?}

C. MARQUES ${ }^{1}$, P. Bastos Lima ${ }^{1}$, P. Gouveia ${ }^{2}$, F. Rio $^{1}$

${ }^{1}$ Neuroradiology Unit, Department of Imagiology, Hospitais da Universidade de Coimbra, Coimbra, PORTUGAL, ${ }^{2}$ Neuroradiology Unit, Centro Hospitalar de Coimbra, Coimbra, PORTUGAL

\section{BACKGROUND AND PURPOSE}

Pituitary adenomas represent an extremely common entity, found as "incidentalomas" in about $40 \%$ of healthy individuals. Being the most common tumor of the sellar region, it also comprises 10 $15 \%$ of all intra-cranial tumors. MRI constitutes the ideal diagnostic imaging modality, allowing adenomas detection and their accurate characterization. The purpose of this study is to characterize the type of adenomas found, in what concerns size, hormonal production and appearance on MRI.

MATERIALS AND METHODS

Retrospective review of clinical files and MRI images of all the patients, who had a histological diagnosis of pituitary adenomas during the years of 2006 and 2007, in this hospital.

RESULTS

A total of 34 patients, with a histological diagnosis of pituitary adenoma, were investigated. Non-secreting macroadenomas constituted the most frequent group of adenomas (61.8\%), mostly with homogeneous isointensity in $\mathrm{T} 1$ (47.6\%), showing high intensity in T2 (10 out of 14 patients with this sequence). They enhanced moderately and in a homogenous way in $33.3 \%$ of the cases. Adrenocorticotropic hormone (ACTH)-secreting adenomas were the second most common group, affecting 5 patients $(14.7 \%)$, with 3 of them being microadenomas. Most of these were isointense in T1, enhancing less than the gland. No prolactinomas were detected.

\section{CONCLUSIONS}

According to what was expected, non-secreting macroadenomas were the most common type of adenomas found. Regarding the hormonally active group, in contrast to literature descriptions, ACTH-secreting constituted the most frequent type instead of prolactinomas. This can be explained by an increasing attempt to treat prolactinomas conservatively, through drug therapy, not obtaining thus a histologic result. T2 sequence seems to have some importance in the non-secreting macroadenomas, since their frequent hiperintensity may be due to the presence of cystic lesions, which can be clinically relevant especially if surgical treatment is performed.

\section{P3:67}

\section{SAFETY OF GADOBENATE DIMEGLUMINE FOR CONTRAST-ENHANCED MR IMAGING OF THE CNS IN INTRAINDIVIDUAL CROSSOVER STUDIES}

\author{
M. KUHN ${ }^{1}$, H. ROWLEY ${ }^{2}$, C. COLOSIMO $^{3}$, M. ESSIG ${ }^{4}$,

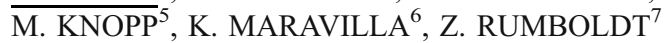 \\ ${ }^{1}$ University of Illinois at Peoria, Peoria, IL, USA, ${ }^{2}$ University of \\ Wisconsin, Madison, WI, USA, ${ }^{3}$ Catholic University of Sacred \\ Heart, Rome, ITALY, ${ }^{4}$ German Cancer Research Center, Heidel- \\ berg, GERMANY, ${ }^{5}$ Ohio State University, Columbus, OH, USA, \\ ${ }^{6}$ University of Washington, Seattle, WA, USA, ${ }^{7}$ Medical Univer- \\ sity of South Carolina, Charleston, NC, USA
}

\section{PURPOSE}

To summarize safety results from 5 prospective, randomized, double-blind, intraindividual crossover comparisons of gadobenate dimeglumine (Gd-BOPTA) with comparator agents for MRI of the CNS.

METHODS

In 5 studies, adult patients underwent 2 identical MRI examinations within $48 \mathrm{~h}$ to 2 weeks, one with $0.1 \mathrm{mmol} / \mathrm{kg}$ bodyweight gadobenate dimeglumine $(\mathrm{N}=378)$ and the other with an equal dose of comparator (gadopentetate dimeglumine [Gd-DTPA, N=224], gadodiamide [Gd-DTPA-BMA, N=125], or gadoterate meglumine [Gd-DOTA, N=31]). Safety monitoring included vital signs, lab values, and adverse events (AE). All patients were monitored for $\mathrm{AE}$ from the time informed consent was obtained until $24 \mathrm{~h}$ after administration of the first contrast agent, and then again from the time the second agent was administered until $24 \mathrm{~h}$ after administration. AE were classified as either serious (ie, death, life-threatening, requiring or prolonging hospitalization) or not serious (rated as mild, moderate, or severe), and the perceived relationship to the contrast agent was noted as probable, possible, not related, or unknown.

\section{RESULTS}

In all trials, the contrast agents were well tolerated, and no significant or clinically meaningful differences between GdBOPTA and comparator were noted in terms of AE incidence or other safety parameters. Based on a pooling of the data from the 5 studies, the overall incidence of adverse events was 42/378 (5.5\%), with an incidence of 25/378 (6.6\%) with Gd-BOPTA, $15 / 224(6.7 \%)$ with Gd-DTPA, $1 / 31$ (3.2\%) with Gd-DOTA, and $1 / 125(0.8 \%)$ with Gd-DTPA-BMA. No potentially-related serious $\mathrm{AE}$ were reported after any contrast agent and all AE potentially related to the agents were mild and self-resolving, except for 5 instances of nausea, headache, pruritus, epistaxis, and ear discomfort of moderate intensity. 


\section{CONCLUSIONS}

Based on intraindividual crossover comparisons involving over 350 patients, the safety of gadobenate dimeglumine is comparable to that of other gadolinium agents used for enhanced MRI of the CNS.

\section{P3:68}

\section{CENTRAL PONTINE MYELINOLYSIS: CT FEATURES}

P. KRANIOTIS, P. ZAMPAKIS, A. KARATZAS, E. KONSTANTATOU, C. KALOGEROPOULOU, T. PETSAS UNIVERSITY HOSPITAL OF PATRAS-RADIOLOGY DEPARTMENT, PATRAS, GREECE

\section{PURPOSE}

Central pontine myelinolysis is a rare disease, due to osmotic demyelination. Its incidence is estimated between 1.2-3.8\%. In liver transplantation patients its incidence rises to $29 \%$. It happens also to alcoholics and patients with rapidly corrected hyponatremia. Greatest risk is when correction rate $>10-15 \mathrm{mmol} / \mathrm{l} /$ day and symptoms occur within 7 days. Our purpose is to highlight the CT features for the diagnosis of this entity which should not be confused with an infarct.

METHODS

A 55 year-old female patient was referred for a routine brain CT for further evaluation due to fever and seizures. The patient had a history of alcohol abuse. The posterior fossa was scanned with $2.5 \mathrm{~mm}$ sections.

\section{RESULTS}

CT depicted a triangular shape hypodense lesion in a central pontine location, characteristically sparing the periphery. The lesion was attributed to central pontine myelinolysis.

CONCLUSIONS

Although MRI is the examination of choice for the brainstem, radiologists can come across central pontine myelinolysis as an incidental finding on CT, when it is established. One should be familiar with the typical features of this rare entity and not confuse it with a pontine infarct.

\section{P3:69}

\section{BRAIN METASTASES: VALUE OF CT-GUIDED BIOPSY OF THORACIC, LIVER, BONE OR ADRENAL CONCOMITANT LESIONS}

E. KOLLIAKOU $^{1}$, P. MANIATIS ${ }^{1}$, C. Triantopoulou ${ }^{1}$, I. Siafas ${ }^{1}$, E. Spathi $^{2}$, D. Apessou ${ }^{3}$, J. Papailiou ${ }^{1}$

${ }^{1}$ Konstantopouleio General Hospital-Department of Computed Tomography, Nea Ionia, Athens, GREECE, ${ }^{2}$ Konstantopouleio General Hospital-Department of Cytology Department, Nea Ionia, Athens, GREECE, ${ }^{3}$ Konstantopouleio General Hospital-Department of Histopathology Department, Nea Ionia, Athens, GREECE

\section{PURPOSE}

To estimate the value of CT guided FNA or FNB of extracranial lesions in order to evaluate the histopathology of the neoplasm in cases of brain metastases.
PATIENTS-METHODS: 50 patients, 41 men and 9 women, 4082 years old who presented with brain metastases from primary lung cancer were enrolled in the study. In order to evaluate the exact histopathology of the primary lesion, 32 patients underwent CT-guided lung biopsy. In 9 out of 18 who had also liver metastases liver biopsy was performed, in 6 out of 9 bone biopsy was done in metastatic sites, while in the rest 3 patients biopsy of metastatic adrenal lesions was performed. Overall 19 FNAs and 31 FNBs were done.

RESULTS

CT-guided FNA or FNB was successful in 40 out of 50 cases, resulting in the diagnosis of 12 small cell carcinomas and 28 adenocarcinomas. All patients were treated accordingly. In 5 cases of lung biopsies, in 2 liver biopsies, in 2 bone biopsies and in one adrenal biopsy exact characterization of tumor type was not possible. CONCLUSION

Characterization of tumor type in cases of metastatic lung cancer has a great impact in proper patient management. CT guided FNA or FNB of extracranial lesions was proven effective in $80 \%$ of the cases obviating the need of complex procedures such as stereotactic brain biopsies.

\section{P3:70}

\section{A CASE REPORT OF AN OSTEOGENESIS IMPERFECTA TYPE I PATIENT WITH PROGRESSIVE LOWER LIMB WEAKNESS ATTRIBUTED TO A MENINGIOMA}

\author{
$\underline{\text { A. ANASTASIOU }}^{1}$, O. KIRITSI ${ }^{1}$, C. TSONIDIS $^{2}$, \\ N. VOUGIOUKLIS ${ }^{1}$ \\ ${ }^{1}$ RADIOLOGY DEPARTMENT HIPPOKRATIO GENERAL \\ HOSPITAL, THESSALONIKI, GREECE, ${ }^{2}$ NEUROSURGERY \\ CLINIC HIPPOKRATIO GENERAL HOSPITAL, THESSALONIKI, \\ GREECE
}

\section{PURPOSE}

We present a case of a 46 year old male with a well known history of type I Osteogenesis imperfecta, with short stature, blue sclera and progressive lower limb weakness.

\section{METHODS}

After clinical examination brain CT was obtained in a spiral CT unit. In addition MR imaging was performed on a 1.5-T unit using standard head coil. Conventional MR with spin-echo T1-weighted images, fast spin-echo T2-weighted images, enhanced spin-echo T1weighted images and Diffusion- weighted images were obtained. Apparent diffusion coefficient (ADC) map was generated with dedicated software.

RESULTS

The CT and MRI images of the brain revealed a large transhemispheric vigorously enhancing extra-axial mass with intraosseous component as well as multiple scattered foci of infiltration in the diploe. The former was considered to be a meningioma and the latter were attributed to the underlying disease. The lesion was totally excised and the histological report confirmed the diagnosis of an atypical type II meningioma with intraosseous infiltration. After the operation the patient regain gradually his muscle power. CONCLUSIONS

In conclusion in an Osteogenesis imperfecta patient the presence of neurologic deficits should prompt the diagnosis of an intracranial tumour even though the concurrence is considered to be extremely rare. 


\section{P3:71}

\section{IMAGING FINDINGS IN TARSAL TUNNEL SYNDROME}

$\frac{\text { P. BRESTAS }}{1}{ }^{1}$, V.K. KATSAROS ${ }^{2}$, M. TSOUROULAS ${ }^{1}$, ${ }^{1}$ ATHENS GENERAL HOSPITAL, DEPARTMENT OF MODERN IMAGING MODALITIES, ATHENS, GREECE, ${ }^{2}$ IKA ONCOLOGY HOSPITAL, DEPARTMENT OF CT AND MRI, ATHENS, GREECE

\section{PURPOSE}

To present the imaging findings in the diagnosis of tibial nerve entrapment in the tarsal tunnel.

\section{MATERIALS AND METHODS}

Twenty patients with clinical and electromyographical findings suggestive of tarsal tunnel syndrome were studied. Dynamic ultrasonographic assessment of the tarsal tunnel and the adjacent anatomic structures was performed using high-frequency transducers. MRI was obtained, including axial, sagittal and coronal T2Weighted, proton density, STIR and T1-Weighted images, before and after administration of Gd-chelate $(0.2 \mathrm{ml} / \mathrm{kg}$ body weight $)$.

\section{RESULTS}

Two cases of muscular anatomical variants were demonstrated. In three cases arthrosynovial cysts caused tibial nerve displacement. Pressure of the tibial nerve also derived in two patients by tenosynovial effusion of the flexor hallucis longus, in one case by venous varicosities and in one case by post-traumatic bone spur. Six cases of direct post-traumatic nerve lesions were also imaged. In the rest five cases nerve involvement was caused by extrinsic or intrinsic space-occupying lesions (lipoma, Schwannoma, neurinoma or sarcoma).

\section{CONCLUSION}

Combination of dynamic ultrasound imaging and MRI of the tarsal tunnel is of outmost importance, in order to discriminate the etiopathology of tibial nerve entrapment.

\section{P3:72}

\section{NEUROIMAGING IN MICROVASCULAR DECOMPRESSION OF THE TRIGEMINAL NERVE}

V. KATSAROS ${ }^{1}$, T. Mpouras $^{1}$, G. Stranjalis ${ }^{1}$, T. Flaskas ${ }^{1}$, N. Bondozoglou $^{2}$, D. E. Sakas ${ }^{1}$

${ }^{1}$ Evangelismos Hospital, Department of Neurosurgery, University of Athens Medical School, Athens, GREECE, ${ }^{2}$ Athens Medical Center, Athens, GREECE

\section{PURPOSE}

To compare neuroimaging and neurosurgical findings in patients suffering from drug-resistant pain of the trigeminal nerve.

\section{MATERIALS \& METHODS}

Twenty-five patients underwent microvascular decompression of the trigeminal nerve. Brain MR imaging included axial proton density, T2-weighted and FLAIR, T1-weighted sequences in three planes before and after iv administration Gd-chelate $(0.2 \mathrm{ml} / \mathrm{kg}$ body weight), as well as $3 \mathrm{D}$ constructive interference steady state sequence (CISS $-0.5 \mathrm{~mm}$ slice thickness) in the posterior cranial fossa. All of the patients had a video tape during the neurosurgical approach. Comparison of the MR imaging and surgical findings followed.

RESULTS

Two patients had meningioma of the cerebellopontine angle, one patient arachnoid cyst and one patient epidermoid. From the 21 patients left, 12 had compression of the Vth cranial nerve by the superior cerebellar artery, six showed compression by veins and three demonstrated unusual compression of the Vth cranial nerve by the ipsilateral vertebral artery, the basilar artery and the contralateral superior cerebellar artery. In five of six cases $(83.33 \%)$ with compression of the Vth cranial nerve by veins MR imaging was negative. MR imaging was accurate in 14/15 patients(93.33\%) with compression of the trigeminal nerve by arterial branches.

CONCLUSION

The presurgical neuroimaging control using CISS sequence in patients suffering from drug-resistant pain of the trigeminal nerve is mandatory.

P3:73

\section{USEFULNESS OF SCOUT VIEW OF HEAD CT}

S. KAN , K. Hayakawa

Kitasato University, Sagamihara, JAPAN

\section{PURPOSE}

Scout view is usually used as an aid to locating the scan level. However, scout view can contribute to the reading of brain CT. The purpose of this exhibit is to illustrate the usefulness of scout view of head CT.

MATERIALS \& METHODS

When reading the brain CT scans, scout view is also viewed and evaluated.

\section{RESULTS}

Following conditions are visualized on scout view and useful information are also obtained.

1. Post operative state

Several types of operation such as burr hole, burr hole button, cranioplasty, aneurysm clipping, deep brain stimulation, VP shunt. craniectomy, craniotomy, coiling for aneurysm

2. Bone defect

Metastasis, diploic vein, vascular groove

3. Bone sclerosis

Metastasis, osteopetrosis, hyperostosis frontalis interna

Fibrous dysplasia

4. Skull fracture

5. Calcification

Cranipharyngioma, AVM, meningioma, petroclinoid ligament

6. Skull size change

7. Paranasal lesion

Sinusitis, tumor, pneumosinus dilatance

8. Sellar enlargement

9. Craniovertebral junction lesions

Atlantoaxial vertical subluxation

CONCLUSION

We found that a careful study of CT scout view can contributes significantly to the diagnosis of brain CT.

Scout view imaging must be displaced with axial head CT slice and carefully evaluated. 


\section{P3:74}

\section{DIPLOPIA, EXTRA OCULAR MUSCLES DISEASES AND MRI}

\section{F. HERAN, M.F. Lafitte}

Fondation Rothschild, Paris, FRANCE

\section{PURPOSE}

Among the many causes of diplopia, extra ocular muscles (EOM) lesions represent a frequent and challenging diagnosis. This work aims to present a simple and practical classification of these diseases, according to clinical data and MRI aspects.

METHODS

Retrospective study of 350 patients, $67 \%$ women, mean age 52 with pathological EOM, depicted on MRI study. Head coil was used. MRI protocol focused on the orbits and performed eyes shut associated at least coronal $\mathrm{T} 2$ and axial $\mathrm{T} 1$ sequences (slice thickness 2 to $3 \mathrm{~mm}$ ) and in most of the cases coronal T2 with fat suppression, axial and coronal $\mathrm{T} 1$ with gadolinium and fat suppression. Analysis of abnormal muscle size, signal, number and location (ie what muscle, uni or bilateral) as well as of associated abnormalities was performed. Relationship with clinical data was studied.

\section{RESULTS}

A practical classification was established, according to the number of EOM involved, which seemed the main discriminating factor. Involvement of all the EOM was due to metabolic affection, of one or few EOM to Grave disease, non specific myositis, tumour (metastasis, rhabdomyosarcoma), infection (cysticercosis) and of all the OEM in one orbit to venous stasis (dural fistulae, apical mass), infiltration (neurofibromatosis). Differences between theses different pathologies was made according to the shape and the signal of the abnormal muscle.

\section{CONCLUSION}

This classification based on the number and location of pathological EOM seems a simple and easy way to reach the etiology of the disease affecting the muscle.

\section{P3:75}

\section{INTERNAL CAROTID AND IPSILATERAL VERTEBRAL ARTERY AGENESIS COMBINED WITH AORTIC ARCH ANATOMIC VARIATION AND PONTINE CRYPTIC (OCCULT) ANGIODYSPLASIA. IMAGING FINDINGS}

\author{
$\underline{\text { E. Primetis }}^{1}$, K. Palialexis ${ }^{1}$, A. Dalakidis ${ }^{1}$, D. Gargas ${ }^{1}$, \\ N. Deligiorgis $^{2}$, A. Gouliamos ${ }^{1}$ \\ ${ }^{1}$ Aretaieio Hospital, Radiology Department, University of Athens, \\ Athens, GREECE, ${ }^{2}$ Errikos Ntynan Hospital, Neurology Depart- \\ ment, Athens, GREECE
}

\section{BACKGROUND}

Internal carotid agenesis is a rare congenital vascular abnormality, (about 100 cases reported in the literature), first described in 1787 by Todd. Imaging study and confirmation was documented in less than 50 of reported cases, most of them recently described. It involves more often the left internal carotid, in contrast to our case. Most patients were asymptomatic and an adequate blood supply from the contralateral internal carotid was observed. Absence of horizontal petrous segment bony carotid canal at skull base is indicative of the abnormality.

PURPOSE

To demonstrate the imaging finding of computed tomography, magnetic resonance imaging and digital subtraction angiography in a case of right internal carotid and ipsilateral vertebral artery agenesis, combined with aortic arch anatomic variation and occult pontine angiodysplasia.

CASE REPORT

A 40 year old female presented with diplopia and acute onset of headache. The patient had no significant clinical history. The neurologic evaluation revealed paresis of the right abducens nerve and hemiparesis of the right side of her body. CT and MRI revealed a focal hemorrhagic lesion at the right side of the pons on the grounds of Developmental Venous Anomaly (DVA) or mixed vascular malformation (DVA + Cavernous Malformation). In addition absence of internal carotid artery and ipsilateral carotid canal were observed. DSA confirmed right internal carotid absence. Also revealed right vertebral artery agenesis and aortic arch anatomic variation (right subclavian artery, right common carotid artery and left carotid artery originate from common trunk). No intracranial aneurysm or angiodysplasia were observed by angiography.

CONCLUSION

Imaging findings of CT, MR and DSA are diagnostic of internal carotid agenesis. Combination of internal carotid and vertebral artery agenesis, congenital aortic arch variation and pontine cryptic angiodysplasia strongly suggests a generalized vascular developmental abnormality during embryogenesis.

P3:76

\section{INTRACRANIAL HYPOTENSION. CLINICAL AND IMAGING FINDINGS}

E. Primetis $^{1}$, A. Dalakidis ${ }^{1}$, T. Prantzos $^{1}$, K. Palialexis ${ }^{1}$, N. Deligiorgis ${ }^{2}$, A. Gouliamos ${ }^{1}$

${ }^{1}$ Aretaieio Hospital, Radiology Department, University of Athens, Athens, GREECE, ${ }^{2}$ Errikos Ntynan Hospital, Neurology Department, Athens, GREECE

\section{BACKGROUND}

Intracranial hypotension is a clinical entity first described in 1953 by Schaltenbrand as spontaneous onset of headache dependent on patient's stature, nausea, neck rigidity and dizziness in patience with low cerebrospinal fluid (CSF) pressure. Diagnosis is based on history, clinical symptoms, imaging and laboratory findings. PURPOSE

To present the imaging findings of intracranial hypotension in Cervical Spine MRI and demonstrate the contribution of imaging in differential diagnosis.

CASE REPORT

A 48 year old man that suffered a stroke without known predisposing factors, presented for scheduled neurologic work up. Immediately after a strenuous and unsuccessful lumbar puncture, the patient complained of headache, neck pain and burning sensation in cutaneous distribution of $\mathrm{C} 6$ nerve root. MRI 
performed the next day, showed in the anterior epidural space a tumourlike lesion with intense enhancement and moderate mass effect on the dural sack. The interpreting radiologist in the outside institution included in the differential diagnosis the likelihood of meningioma and infection but not epidural venous plexus expansion. Patient's symptoms subsided 10 days after. The following lumbar puncture showed no pathologic findings except for slight protein increase in CSF. CSF pressure wasn't measured. Clinical and laboratory results were unremarkable for malignancy, as well as infection, granulomatous and inflammatory disease. The patient refused surgical exploration. At follow up one year after, MRI yielded normal findings.

In this case, imaging findings as well as history and symptoms evolution were diagnostic of anterior epidural venous plexus expansion due to intracranial hypotension secondary to unsuccessful lumbar puncture.

\section{CONCLUSION}

Venous plexus expansion must be included in the differential diagnosis of tumorlike lesions in the anterior epidural space. Knowledge of its characteristic imaging appearance is critical for recognition of this entity and discriminate it from tumours and infectious or inflammatory diseases, in order to avoid misinterpretation and unnecessary interventions and treatment.

\section{P3:77}

\section{VENTRICULUS TERMINALIS}

S. COSTA PEREIRA, G. Santos, T. Parreira, D. Marnoto, E. Machado, O. Brito

University Hospital of Coimbra - Neuroradiology, Coimbra, PORTUGAL

\section{INTRODUCTION}

Ventriculus terminalis (VT) designates a dilated cavity at the conus medullaris. This rare developmental defect, almost exclusively found in children, usually represents an incidental finding, although it can present clinically as back pain, signs of medullar compression or urinary disturbances. Its etiology remains controversial, being probably related with the persistence of a ventricular structure, the $\mathrm{V}$ ventricle, or resulting from a proliferation of ectopic ependimal cells. CLINICAL CASES

1st case: 45 year-old female patient with unremarkable past medical history. She complained of back pain with bilateral leg irradiation for the last 6 months and denied any trauma. MRI revealed a large cystic dilatation of the conus medullaris, measuring $5.5 \mathrm{~cm}$ (vertical axis), at the L1 to L2 level. The content was homogeneous, with the same signal intensity that CSF in all sequences and no enhancement. There was no medullar edema or any dilatation of the ependimal canal. Management consisted of pharmacologic pain control and periodical imaging surveillance.

2nd case: 51 year-old female patient presenting a 1-year history of back pain with unilateral irradiation to the right leg. She was also being treated for depression and denied traumatic events. MRI revealed a cystic dilatation of the conus medullaris, at D12-L2 levels, displaying similar features to those observed in the first patient. As medical treatment revealed disapointing, a surgical approach was decided. A cyst-subaracnoid derivation was performed, with full recovery over the next 2 months.

\section{CONCLUSIONS}

We report these two cases, presenting large lesions that were managed in different ways and focus attention on the imagiological diagnosis of VT. The differential diagnosis of a cystic dilatation of the conus medullaris includes others disorders, such as a cystic medullar neoplasm and syringomyelia. In this setting, imaging techniques are very useful, as VT almost always presents typical features, allowing accurate diagnosis and preventing unnecessary and potencially harmful invasive procedures.

\section{P3:78}

\section{IMAGING FINDINGS OF VON HIPPEL-LINDAU DISEASE WITH REGARDING OF A FAMILY}

M. APAYDIN, M. APAYDIN, M. VARER, A. SARSILMAZ, N. ERDOGAN, E. Uluc, O. Oyar

IAEAH-Department of Radiology, IZMIR, TURKEY

Von Hippel Lindau syndrome(vHL'S) is an autosomal dominant multisystem/multitumoral cancer disease diagnosed by clinical, radiologic and genetic findings. It's prevelance has been estimated to be of $1 / 36000$. The tumors can be benign or malignant. Central nervous system lesions include hemangioblastomas and endolymphatic sac tumors. Visceral manifestations include renal/pancreatic carcinomas and cysts, neuroendocrine tumors and epipididimal cysts. There is germline mutation of the VHL tumor suppressor gene on the short arm of chromosome 3p25-26. Symptoms caused by vHL'S depend on the organ involved. Patients with involvement of the CNS at presentation are usually aged 25-35 years. CNS hemangioblastoma is the most commonly recognized manifestation of vHL'S and occurs in $40 \%$ of patients. The most important mortality factors are renal cell carsinoma and cerebellar hemangioblastomas. We represent a family with vHL'S and discuss imaging findings with regards to the literature. Radiologic imaging is very important for the early diagnosis and treatment especially for asymptomatic patients.

\section{P3:79}

\section{EVIDENCE OF VASCULAR COMPROMISE OF VISUAL CORTEX IN MIGRAINOUS HEADACHE: A CASE REPORT OF MRI STUDY}

\section{O. CHAWALPARIT ${ }^{1}$, W. Siriacharwattana ${ }^{2}$}

${ }^{1}$ Department of Radiology, Faculty of Medicine Siriraj Hospital, Mahidol University, Bangkok, THAILAND, ${ }^{2}$ Neuroscience Center, Bangkok Hospital, Bangkok, THAILAND

\section{OBJECTIVE}

To demonstrate MRI evidence of vascular compromise of a 17-years old female presenting with migrainous headache.

METHOD

A 17-years old female presented with migrainous headache for 2 days. She had visual aura lasting for a few hours before the attack. She went to see the physician with the symptom of left sided numbness during the headache. No neurological deficit was 
detected when the first MRI was performed. All of her symptoms resolved after 4 days of the attack.

\section{RESULT}

The brain MRI study showed focal area of restricted diffusion at right visual cortex. Short segment of vascular enhancement is noted on the surface of the affected gyrus. MR spectroscopy showed normal NAA, Cho, Cr with no elevation of Lac. Follow up MRI study next 5 months showed normal finding with no residual lesion.

\section{CONCLUSION}

The authors concluded that abnormality on first MRI was the ischemic insult of the ictal visual cortex which was transient during migraine attack. The pathophysiology was more likely from reversible focal venous congestion.

\section{P3:80}

\section{WEB-BASED TUTORIAL OF EAR IMAGING ANATOMY}

G. Sparacia, P. Purpura, G. Cavarretta, M. Midiri

DI.BI.MEL, Sezione di Radiologia P Cignolini, Palermo, ITALY

\section{PURPOSE}

Imaging plays a central role in the management of ear's disease because therapeutic approach substantially depend on extension of lesions and involvement of different structures inside ear. The purpose of this exhibit is to develop an interactive tutorial software for understanding the ear anatomy by $\mathrm{CT}$ and MR imaging.

METHOD AND MATERIALS

This tutorial is based on a web interface. The user can learn ear anatomy based on of CT and MR images in axial, coronal and sagittal sections and with multiplanar reconstructions or $3 \mathrm{D}$ reconstruction of CT images.

\section{RESULTS}

Tutorial is made up of CT and MR images in axial, coronal and sagittal sections and with multiplanar reconstructions or 3D reconstruction of CT images. Web interface is organized in frames and each section has been developed following the WBC guidelines for web content accessibility. By using a mouse to select specific portions on CT and MR images the user can learn normal anatomical structure inside ear. CONCLUSION

This interactive tutorial software should be a quick and simple instrument to identify normal anatomical structures inside ear in $\mathrm{CT}$ and MR imaging.

\section{P3:81}

\section{MR AND CT EVALUATION OF CONGENITAL ANOSMIA TESTED WITH SEMIQUANTITATIVE OLFACTOMETRY}

A. MAS BONET ${ }^{1}$, R. SOLER VILLARASA ${ }^{2}$, M.J. PICADO ${ }^{1}$, A. MOLL SERVERA ${ }^{1}$, G. ALONSO FIEL ${ }^{1}$, L. NOJE ${ }^{1}$

${ }^{1}$ Section of Neuroradiology. Hospital Son Dureta, PALMA DE MALLORCA., SPAIN, ${ }^{2}$ Department o ENT, PALMA DE MALLORCA, SPAIN

\section{PURPOSE}

Anosmia since birth or early childhood could be associated with chromosomal disorders or without evidence of other defects (isolated anosmia). Only a few studies we have found published about the fronto-basal anomalies associated to this entity. The purpose of the present study is to describe the morphologic anomalies of the frontal lobe, olfactory tracts and anterior fosa associated to this anomaly.

METHODS

All the patients presented were referred from the ENT department with complete anosmia tested by semi quantitative olfactometry. Eighteen patients (10 females and eight males) were evaluated, ten by using 1,5-T system Siemens Magnetom Vision and eight by Siemens Avanto.In all we also evaluated the paranasal sinuses and the anterior skull base.by using 64row Multidetector CT,

\section{RESULTS}

Six patients had bilateral hypoplastic olfactory bulbs. Two patients had hypoplastic olfactory bulbs in the right and aplastic in the left. One hypoplastic in the left and aplastic on the right. Five had bilateral aplastic olfactory bulbs. Another one had Kallmann's syndrome. In four patients none anomalies were detected. All the patients with olfactory bulbs anomalies had associated smaller olfactory sulcus and dysplastic girus rectus. In four of them the fronto-basal frontal lobe had and appearance in pseudomeningocele with descends and flattening anterior skull base better detected by CT. CONCLUSIONS

Anomalies of the olfactory tracts, girus rectus and olfactory sulcus are common anomalies associated to congenital anosmia. Only in four cases we detected associated osseous anomalies of the anterior skull base.

\section{P3:82}

\section{THE OLYMPIC ALGORITHM: UTILISATION AND PRELIMINARY EVALUATION OF A NEW STRATEGY IN DIAGNOSIS OF THE MIDFACE}

\author{
J. ZAJACZEK, F. DONNERSTAG, K. BLUM, P. OPHERK, \\ W. SCHLÖTELBURG, H. LANFERMANN \\ Inst. for Diagnostic and Interventional Neuroradiology, Hannover \\ Medical School, Hannover, GERMANY
}

Complex threedimensional anatomical relationships within the midface lead to difficulties in detection of pathology. The majority of existing schematisations or classification systems, particularly for midfacial fractures, are very complex and not everywhere part of daily routine. To overcome this diagnostic challenge the diagnostic strategy of the "Olympic rings of attention" has been introduced previously. Now this pragmatic pictogram is integrated into daily practice and initial experience is evaluated to estimate its usefulness. Case histories of midfacial fractures with varying complexity were analysed by 3 radiologists in training. Analysis was split, at first investigators attention was focussed on the concept of beams and buttresses that are most relevant in examination of the midface. 10 cases had to be analysed, completion and quality of report on diagnostic findings were evaluated. After 3 weeks same radiologists were made familiar with the concept of the "Olympic rings of attention". 10 different cases had to be analysed by harking back to this diagnostic strategy. Again evaluation of actual benefit was performed, including standardised assessment sheet and interview. 
All investigators rated the diagnostic strategy as very beneficial in aspects of certainty and complete acquisition of diagnostic findings, especially if reflecting on long range effects of its internalisation. Utilisation of the diagnostic strategy is appreciated to be beneficial within night duty in particular.

Consolidated analysis of the assessment sheets shows that diagnostic report is prepared faster, more comprehensive and more compactly with the help of the diagnostic strategy of the "Olympic rings of attention" overall.

Preliminary evaluation demonstrates that our diagnostic strategy helps to increase quality of radiological report particularly if reflecting on comprehensive coverage of pathology, reliability, and time management.

The new diagnostic strategy is promising not only for daily routine but also for educational purposes. To facilitate recognition it shall be denoted as the "Olympic algorithm" within midfacial diagnostics henceforward.

\section{Head and Neck Imaging and Intervention}

\section{P4:83}

\section{NEW CONCEPT OF HEAD AND NECK VASCULAR TERRITORY ACCORDING TO THE FUNCTIONAL VASCULAR LAYER}

Y. UCHIYAMA, T. ABE, N. TANAKA, M. TSUJI, N. HAYABUCHI KURUME UNIVERSITY SCHOOL OF MEDICINE, FUKUOKA, JAPAN

\section{OBJECTIVE}

To understand the three dimensional vascular territory of the external carotid artery and its branches.

METHODS

To determine the vascular territory of the head and neck, functional layers based on the anatomical functional concept with hemodynamic balance were described. Anatomical findings obtained from digital subtraction angiography (DSA) and flat panel detector computed tomography (FPD-CT) with super selective injection of contrast material, and 3-D CT angiography were evaluated.

\section{RESULTS}

The four functional vascular layers were ideally reconstructed. The musculo-cutaneous layer was fed by facial artery, superficial temporal artery, and occipital artery. The neuro-meningeal layer was fed mainly by middle meningeal artery, ascending pharyngeal artery and their anastomosis. The naso-masticatory layer was fed by maxillary artery and its branches. The oro-laryngeal layer was fed by lingual artery, superior thyroid artery and their branches and collateral circulations. These four layers had collateral network formation each other. These functional vascular layers were superimposed on the CT image for easy understanding of the vascular territory in the head and neck.

\section{CONCLUSION}

The concept of functional vascular layer makes it easy to understand the vascular territory of external carotid artery and its branches. This will be effective for understanding blood supply of the tumors or vascular malformations, especially for safety interventional radiological procedure in this region.

\section{P4:84}

\section{CALVARIAL LESIONS: CT AND MRI FINDINGS}

\author{
M. THEOFANOPOULOU $^{1}$, I. NIKAS ${ }^{2}$, K. SIRGIANNIS ${ }^{2}$, \\ V. THEODOROPOULOS ${ }^{2}$ \\ ${ }^{1}$ IMAGING DPT, IONIA EUROMEDICA DIAGNOSTIC \\ CENTER, ATHENS, GREECE, ${ }^{2}$ IMAGING DPT, AGIA SOFIA \\ CHILDREN'S HOSPITAL, ATHENS, GREECE
}

\section{PURPOSE}

Skull vault lesions are common and they may be clinically identified as palpable masses or incidentally encountered in radiologic examinations. Their differential diagnosis which often presents difficulties is important in order to decide further management: biopsy, surgical intervention or follow-up. Purpose of this study is to present a review through imaging of the most frequently encountered calvarial lesions. METHODS

Lesions included in our presentation occurring in the pediatric as well as the adult population, are categorized as tumoral and nontumoral

\section{RESULTS}

Plain radiographs are usually the first imaging approach in evaluating lesions of the cranial vault. In infants US is often used to further characterize the lesion. CT is able to evaluate localization (inner or outer table), lytic or sclerotic patterns, contour of the lesion and the presence of calcifications, whereas MR is better at delineating soft-tissue involvement, bone marrow abnormalities and extention into the intracranial cavity. The most commonly encountered lesions in children and young adults were: cephaloceles, dermoids, epidermoids, $\mathrm{LCH}$, , angiomas, fibrous dysplasia, osteomas and sinus pericranii. In adults metastases and multiple myeloma were more often found.

\section{CONCLUSIONS}

Imaging is essential in achieving a correct diagnosis or limiting the differential diagnosis of most calvarial lesions, thus aiding in their further management.

\section{P4:85}

\section{POSTTRAUMATIC CERVICAL SPINE INJURIES-5-YEAR REVIEW}

N. SYRMOS, C.H. ILIADIS, G. GAVRIDAKIS, K. GRIGORIOU, D. ARVANITAKIS, L. TRIANTAFYLLOU

NEUROSURGICAL DEPARTMENT-CT DEPARTMENT VENIZELEIO HOSPITAL, HERAKLION, CRETE, GREECE

\section{AIM}

The purpose of this study was the epidemiological analysis of trauma causes and trauma effects including age and sex.

MATERIAL-METHODS-During the last five years (2003-2008) 138 patients after cervical spine trauma were studied retrospectively. The following parameters were estimated: 1.age, 2. sex ,3. cause of injury,4. type and localization of pathology.

\section{RESULTS}

The mean age was 32.5 years. The largest age group consisted of patients 25-35 years old. Males predominated over females in all age groups. The most common cause of cervical spine injury was 
traffic accident with a patient as a car driver or as a car passenger$50 \%$ - Luxations and subluxations were most often localized on C1-C2 level and on C5-C6 level. Fractures were most often localized in $\mathrm{C} 5$ vertebra.

\section{CONCLUSION}

The results of this study underline the importance of proper clinical and computed tomographic evaluation in cases of facial fractures for recognition of cervical spine trauma Different approaches and measures shall be taken in regard to different age groups, different regions, different times and different transport modes, and corresponding policies shall be adopted.

\section{P4:86}

\section{MANAGMENT OF PATENTS WITH SEVERE HEAD TRAUMA-WITH SERIAL COMPUTED TOMOGRAPHY IMAGING}

N. SYRMOS, G. GAVRIDAKIS, C.H. ILIADIS, K. GRIGORIOU, D. ARVANITAKIS, L. TRIANTAFYLLOU

NEUROSURGICAL DEPARTMENT-CT DEPARTMENT VENIZELEIO HOSPITAL, HERAKLION, CRETE, GREECE

\section{AIM}

aim of our study was to evaluate the risk of progression of traumatic intracranial lesions in patients with severe head trauma by comparing initial and subsequent computed tomography (CT) scans.

MATERIAL-METHODS

We performed a retrospective study in 70 patients youngerwho underwent repeated CT scanning within 24 hours of their initial CT scanning procedure. The risk of progression between the initial and repeated CT scanning sessions and the need for delayed neurosurgical intervention were determined for each lesion type. RESULTS

In 25 patients $(35,7 \%)$ the normal findings on the initial CT study did not change on subsequent imaging. In 45 patients in whom abnormal findings were present on the initial scan, progression was demonstrated. Patients with epidural hematoma ,subdural hematoma cerebral edema and intraparenchymal hemorrhage were found to be at a significantly increased risk for progression and to require delayed neurosurgical intervention .No significantly increased risk was found for patients with subarachnoid hemorrhage ,intraventricular or skull fracture.

CONCLUSIONS

Repeated CT imaging in patients with high-risk lesions such is recommended.. CT remains to be the imaging modality of choice in the evaluation of patients with acute head trauma

\section{P4:87}

\section{MDCT ANGIOGRAPHY FOR THE DIAGNOSIS OF CAROTID AND VERTEBRAL ARTERY DISSECTIONS}

P. Zampakis, S. Spiliopoulos, P. Kraniotis, O. Romanos, A. Diamantopoulos, T. Petsas, C. Kalogeropoulou University Hospital of Patras, Patras, GREECE

\section{PURPOSE}

To present the reliability of multi-detector computed tomography angiography(MDCTA), for the diagnosis of cervical arteries dissections.

MATERIALS AND METHODS

Between 2006 and 2008 in total 7 patients ( 5 men) with a mean age of 50 years (range 45-55), without any previous medical history, were presented with the clinical diagnosis of stroke, confirmed by plain CT. Our department's imaging protocol in order to investigate the cause of the underlying pathology includes a MDCTA of the carotid and vertebral arteries. Three patients were followed-up for a mean time of one year. RESULTS

In 6 cases MDCTA revealed the pathognomonic signs of the carotid artery dissection as the cause of the ischemic stroke (increase of the vessel diameter, multiple stenosis/occlusions, increase of the vessel wall diameter) and in the remaining case a vertebral artery dissection as the cause of the subarachnoid hemorrhage. In 3 cases the diagnosis was confirmed by MRI. TUS revealed two cases of stenosis/ occlusion of the internal carotid artery and in one case thrombus in the carotid artery. One patient died from subarachnoid hemorrhage while the remaining 6 had a relatively favorable outcome with variable neurological defects.

CONCLUSIONS

MDCTA is a very effective non invasive method for the detection of carotid and/or vertebral artery dissection, as it can detect the pathognomonic signs of this syndrome and pose immediate diagnosis with accuracy.

\section{P4:88}

\section{MAGNETIC RESONANCE IMAGING WITH CINE PHASE-CONTRAST: APPLICATIONS TO CEREBROSPINAL FLUID FLOW STUDIES}

\author{
S. SOTO $^{1}$, F. ESTEFANIA ${ }^{1}$, H. PULGAR ${ }^{1}$, M.C. VIAL ${ }^{2}$, \\ V. VILLALON ${ }^{1}$ \\ ${ }^{1}$ CLINICA DAVILA, SANTIAGO, CHILE, ${ }^{2}$ OXFORD UNIVER- \\ SITY, OXFORD, UNITED KINGDOM
}

\section{PURPOSE}

To assess the usefulness of cine phase-contrast magnetic resonance imaging (MRI) to the evaluation of cerebrospinal fluid (CSF) flow abnormalities.

METHODS

Examinations using cine phase-contrast can be analyzed in a quantitative or qualitative fashion. The former can be used to study CSF flow dynamics in the aqueduct of Sylvius or to evaluate fluid flow restoration after ventriculostomy. Both quantitative and qualitative phase-contrast applications to CSF flow are evaluated, and substantiated by examples.

RESULTS

Different pathologies that compromise CSF flow are depicted along their phase-contrast imaging characteristics. Inherent to idiopathic normal pressure hydrocephalus is aqueductal flow void sign, as also are, specific CSF flow dynamics in the aqueduct of Sylvius, such as: maximum diastolic and systolic velocity, mean velocity, maximum diastolic and systolic blood flow, mean flow and stroke volume. In the case of endoscopic third 
ventriculostomy the quantification of stroke volume is a valid indicator of the post-operative functional status. Furthermore, phase-contrast MRI can be applied to the study of communications between intra-cranial arachnoid cysts and cisterns, and to the analysis of intra-ventricular lesions.

CONCLUSION

Either quantitative or qualitative cine phase-contrast MRI studies of CSF flow represent an invaluable assistance to the diagnosis of specific clinical entities.

\section{P4:89}

\section{ENDOVASCULAR TREATMENT OF HIGH-FLOW CERVICAL VASCULAR ANOMALIES: CASE REPORT}

\section{B. PRSTOJEVIC, I. VUKASINOVIC}

Institute of Radiology-Department of Neuroradiology, Belgrade, SERBIA

\section{PURPOSE}

High-flow cervical vascular anomalies, arteriovenous malformations (AVM) and arteriovenous fistulas (AVF) are rare pathological conditions, with congenital, spontaneous and posttraumatic etiology. Cervical AVF are consisted of high flow arteriovenous shunt between external carotid artery (ECA) and internal jugular vein (IJV).

\section{METHODS}

We present you a case of 3 year old male with pulsatile mass, thrill and bruit on the right side of the neck, later revealed on digital subtraction angiography (DSA) as high-flow cervical AVF between right ECA and right IJV. AVF had numerous aberrant draining veins, shunting large amount of blood through the both subclavian veins, and superior vena cava into the heart. Regarding AVF, on examination boy was healthy, demonstrating normal heart function. RESULTS

AVF was completely embolized transarterially with 19 coils, which was accompanied with immediate thrill and bruit disappearance after last coil deposition. As complication we encountered starting of one coil migration in the middle of embolization. Speeding up the coiling procedure we managed to stabilize proximal end of migrating coil attached to the main coil mass. Embolization went without further complications, and boy was discharged in good health. On DSA control AVF remains completely obliterated. CONCLUSIONS

Endovascular embolization is the treatment of choice for highflow cervical vascular anomalies.

\section{P4:90}

\section{SINGLE-CENTER EXPERIENCE IN ENDOVASCULAR TREATMENT OF INTRACRANIAL ANEURYSMS}

$\underline{\text { B. PRSTOJEVIC }}^{1}$, M. SAMARDZIC ${ }^{2}$, V. ANTUNOVIC ${ }^{2}$,

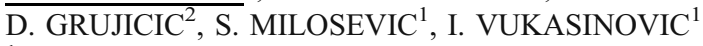

${ }^{1}$ Institute of Radiology-Department of Neuroradiology, Belgrade, SERBIA, ${ }^{2}$ Institute of Neurosurgery, Belgrade, SERBIA

\section{PURPOSE}

Brain aneurysms can cause intracranial hemorrhages with devastating consequences. Advancements in technology are rapidly improving endovascular armamentarium enabling treatment of most complex aneurysms. We present our clinical experience in endovascular treatment of intracranial aneurysms since the very beginning, June 2006.

\section{METHODS}

This is a retrospective review of 62 patients with 70 intracranial aneurysms who underwent embolization between June 2006 and May 2009. In 2006 two, 2007 twenty seven, 2008 twenty one, and 2009 twenty aneurysms were embolized. For the majority of cases, indication field were complex inoperable aneurysms. Patient age ranged from 24 to 81 years. Aneurysm size (mm) distribution was: 39 small (0-10), 28 big (11-24), and three giant $(>=25)$. Three were treated during the acute, and 40 during chronic phase of subarachnoid hemorrhage. Embolization was performed with coils in all aneurysms but one, which was embolized with liquid embolic material Onyx.

\section{RESULTS}

Angiographic results revealed complete occlusion in 29 of 70 aneurysms (41.4\%), neck remnants in $16(22.9 \%)$, and incomplete occlusion in $25(35.7 \%)$. Two of the 62 patients $(3.2 \%)$ experienced neurological deterioration caused by immediate procedural complications, which later completely resolved. Two patients died $(3.2 \%)$, first one as a result of aneurysm rerupture minutes before patient was put under general anesthesia, and second one because of spontaneous right middle cerebral artery thrombosis during procedure.

\section{CONCLUSIONS}

Experience in endovascular techniques, with careful consideration of vascular anatomy issues is essential to achieve technical and anatomical success while avoiding complications.

\section{P4:91}

\section{DIFFERENTIATION OF CERVICAL LYMPHADENOPATHY WITH APPARENT DIFFUSION COEFFICIENT VALUE}

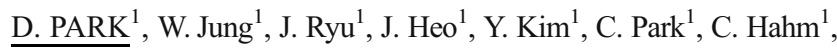 \\ Y. Lee ${ }^{2}$, S. Lee ${ }^{2}$ \\ ${ }^{1}$ Hanyang University Guri Hospital, Guri, SOUTH KOREA, \\ ${ }^{2}$ Hanyang University Hospital, Seoul, SOUTH KOREA
}

\section{PURPOSE}

ADC value for characterizing cervical lymphadenopathy is evaluated.

METHODS

MR imaging was performed in 24 patients(men: women=14: 10; 8 80 years old) with cervical lymphadenopathy, which consisted of 10 patients with squamous cell carcinomas of nasopharynx (5), oropharynx (2) and larynx (3), 1 patient with adenoid cystic carcinoma of oropharynx, 5 patients with non-Hodgkin's (3) and Hodgkin's lymphomas (2), and 8 patients with tuberculosis (2), Kikuchi's disease (1) and other nonspecific inflammation (5). Positive cervical nodes were histopathologically proved in 45 of 49 nodes, and the other 4 metastatic nodes of known primary head and neck cancers were diagnosed by increased FDG uptake on PET. 
ADC values of cervical lymph nodes including 17 metastatic nodes, 16 lymphomatous nodes, and 16 benign nodes were measured with b factor, $800 \mathrm{~s} / \mathrm{mm} 2$. Each group of metastatic, lymphomatous and benign nodes was statistically compared with ADC values.

\section{RESULTS}

The mean ADC values of metastatic, lymphomatous and benign nodes were $0.662 \pm 0.154 \times 10-3 \mathrm{~mm} 2 / \mathrm{s}(\mathrm{n}=17), 0.475 \pm 0.589 \times 10$ $-3 \mathrm{~mm} 2 / \mathrm{s}(\mathrm{n}=16), 0.823 \pm 0.123 \times 10-3 \mathrm{~mm} / \mathrm{s}(\mathrm{n}=16)$, retrospectively. Mean ADC value of malignant nodes including metastatic and lymphomatous nodes, $0.571 \pm 0.150 \times 10-3 \mathrm{~mm} 2 / \mathrm{s}$. Mean ADC value is significantly lower in malignant nodes than benign nodes $(\mathrm{P}<0.001)$, and also significantly lower in lymphomatous nodes than metastatic lymph node $(\mathrm{P}<0.001)$.

The mean ADC value of $0.650 \times 10^{-3} \mathrm{~mm} 2 / \mathrm{s}$, as a cut-off value for differentiating malignant from benign lymph nodes is obtained with the highest accuracy of $83.6 \%, 78.8 \%$ sensitivity, and $93.8 \%$ specificity. And cut-off value of 0.587 X $10-3 \mathrm{~mm} 2 / \mathrm{s}$ for differentiating lymphomatous and metastatic lymphadenopathy is obtained with the highest accuracy of $84.8 \%, 100 \%$ sensitivity, and $70.6 \%$ specificity.

CONCLUSION

ADC value can help the characterization and differentiation of cervical lymph nodes, especially in between benign, metastatic and lymphomatous nodal groups.

\section{P4:92}

\section{AN EXPLORATORY ANALYSIS OF VARIATIONS WITHIN THE NORM OF THE ARTERIES ORIGINATING FROM THE AORTIC ARCH}

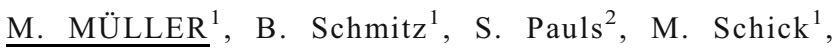 \\ W. Schlötzer \\ ${ }^{1}$ Sektion Neuroradiologie, Klinik für diagnostische und \\ interventionelle Radiologie, Uniklinik Ulm, Ulm, GERMANY, \\ ${ }^{2}$ Klinik für diagnostische und interventionelle Radiologie, Uni- \\ klinik Ulm, Ulm, GERMANY
}

\section{AIM}

Variations within the norm of the aortic arch often appear as secondary finding in routine CT-scans. However, these variations can be an issue when performing diagnostic or interventional angiography. In this study we analysed the common variations found within the arteries originating from the aortic arch.

METHODS

809 of the scans we analysed were carried out in order to inspect the thoracical or cervical arteries. The other 1224 scans were carried out as contrast media assisted routine thoracical examinations. 1200 of the 2033 patients were male, 833 female. The average age was $61 \pm 16$ years. Scans were carried out during $1 \mathrm{st}$ April 2008 to 15th March 2009.

\section{RESULTS}

The total percentage of variations within the analysed patients was $13 \%(270 / 2033)$. In $8 \%(167 / 2033)$ a truncus bicaroticus was found. $4 \%(87 / 2033)$ of the patients showed a left vertebral artery originating directly from the aortic arch, mostly proximal of the left subclavian artery. In one case the left vertebral artery originating directly from the aortic arch departed distal of the left subclavian artery. In $1 \%(20 / 2033)$ we found an arteria lusoria. 6 of the patients showed 2 coexistant variations. We also found one single case of a dexter aortic arch with separate origins of the carotic as well as subclavian arteries.

\section{CONCLUSIONS}

Variations within the norm of the arteries originating from the aortic arch are often found in routine CT-scans. The most common variation is the truncus bicaroticus. Other regularly found variations are a left vertebral artery originating directly from the aortic arch as well as an arteria lusoria. Other variations such as a dexter aortic arch are rare. Before performing diagnostic or interventional angiography the knowlegde of variations within the aortic arch is certainly helpful. Such knowlegde can easily be aquired by CT-scans carried out during routine diagnostic procedures.

\section{P4:93}

\section{CONGENITAL TEMPORAL BONE BILATERAL MALFORMATIONS IN SAETHRE-CHOTZEN SYNDROME}

\section{B. MOREIRA, P. PINTO, D. DIAS}

Hospital Geral de Santo Antonio - Neuroradiology Department, PORTO, PORTUGAL

\section{INTRODUCTION}

Saethre-Chotzen syndrome (acrocephalosyndactyly type III) is characterized by a broad set of malformations. Its estimated prevalence is 1 in 25000 to 50000 live births, and it is transmitted as an autosomal dominant trait with full penetrance and great variability in expression. Like many other syndromes and patterns of malformations, it may have auditory dysfunction as a component.

CASE REPORT

A 7-year-old boy with phenotype characteristics of SaethreChotzen syndrome (including coronal craniosynostosis) and normal developmental milestones, was seen by an otolaryngologist because of bilateral conductive hearing loss. The otologic examination revealed bilateral microtia type I and a membranous atresia in the osseous portion of the external auditory canal (EAC). He was also concerned about ossicular malformations, as they also partially arise from the first branchial cartilage (Meckel's cartilage), and ordered a CT scan. It confirmed the EAC membranous atresia and, although both medial ears were well developed, showed bilateral bony fusion of the incudomalleolar joints and an osseous fixation of both incus to the epitympanum. The other ossicles, the facial nerves and the inner ears were intact and symmetrical.

\section{COMMENTS}

Hearing loss is a variable finding in patients with syndromic craniosynostosis. From a clinical perspective, the findings of imaging studies may have ramifications in the habilitation or rehabilitation choices.

This case serves to reinforce the need for early screening for hearing loss, frequent otological examinations and prompt treatment of disease in children who exhibit craniofacial deformities. 


\section{P4:94}

\section{RECURRENT RETROPHARYNGEAL SPACE PLEOMORPHIC ADENOMA WITH SKULL BASE INVASION AND DISTANT METASTASES}

\section{B. MOREIRA, P. PINTO, C. VASCONCELOS, R. CRUZ}

Hospital Geral de Santo Antonio - Department of Neuroradiology, Porto, PORTUGAL

\section{INTRODUCTION}

Pleomorphic adenomas (PA), are common tumors of salivary glands. Although they are benign tumors, they occasionally recur after surgery and may undergo malignant transformation with potentially devastating consequences. Three types of malignancies are associated with PA: carcinoma ex-pleomorphic adenoma, and rarer variants such as true malignant mixed tumor and metastasizing benign pleomorphic (MPA).

CASE REPORT

A 40-year-old lady presented to the emergency room complaining of left ear pain and of non-specific right brachialgia. She had a previous history of PA of minor salivary gland in the retropharyngeal space diagnosed eleven years before, with two surgeries and post-surgical irradiation, the second one after a local recurrence.

The otologic findings were consistent with a left middle ear infection and the pharyngeal examination revealed a massive swelling of the nasopharyngeal wall.

The MRI confirmed local recurrence of a sharply marginated heterogeneous PA of the retropharyngeal space without invasion of the surrounding cervical tissues but with thinning and erosion of the skull base and extension into the medial fossa and into Meckel's cave and cavernous sinus. The neurologic examination, repeated after this exam, was unremarkable.

It was also noticed a right epidural infiltrating mass centered on C5-C6 with partial destruction of the adjacent vertebral bodies and extension, through an enlarged intervertebral foramen, to the prevertebral space. Coronal STIR sequence showed swelling and edema of the ipsilateral brachial plexus without contrast enhancement, consistent with denervation.

The pathologic studies of both lesions were consistent with a lowgrade salivary gland tumor.

\section{CONCLUSIONS}

Metastasizing pleomorphic adenoma is a rare tumor which etiology remains incompletely understood. This case illustrates an unusual location for the primary tumor and emphasizes the importance of meticulous surgical approach to prevent tumor recurrence and seeding.

\section{P4:95}

\section{BILATERAL PETROUS APEX CEPHALOCELE WITH EMPTY SELLA: A CASE REPORT}

\section{G. LEE, J. LEE, J. SHIM, Y. KIM, H. KIM, J. SEO} SEOUL PAIK HOSPITAL, SEOUL, SOUTH KOREA

The petrous apex can be involved by various cystic and solid lesions. Cystic lesions, such as cholesterol granuloma, mucocele, and congenital or acquired cholesteatoma, are much more common than the solid lesions. Petrous apex cephalocele(PAC) is a rare lesion. It arises from Meckel's cave and secondarily erodes the petrous apex, and is characterized by eccenteric location, cystic appearance, and contiguity to the posterolateral aspect of Meckel's cave on imaging finding. Patients with PAC can present trigeminal nerve symptoms or CSF leak. Primary empty sella is reported in $5-35 \%$ of the general population. PAC and empty sella are similar mechanically in terms of CSF expansion and erosion of adjacent bony structures, so raising a suspicion of possible etiologic relationship between the two.

We exprienced a case of PAC in 44-year-old female patient, who presented tinnitus and left hearing difficulty. Temporal bone CT and MRI including diffusion-weighted sequence demonstrated typical imaging findings at bilateral petrous apex with empty sella. So we describe here imaging findings of CT and MRI with the review of literature.

\section{P4:96}

\section{SUPERSELECTIVE INTRAARTERIAL CHEMOTHERAPY FOR HEAD AND NECK TUMORS: USEFULNESS OF CONE BEAM CT WITH A FLAT PANEL DETECTOR}

\section{Y. KOROGI}

University of Occupational and Environmental Health, Kitakyushu, JAPAN

The purpose is to assess the usefulness of the cone-beam CT using the flat panel detectors (FPD) in conjunction with conventional digital subtraction angiography (DSA) for performing superselective intraarterial chemotherapy for head and neck tumors. Twenty-three consecutive patients (43 feeding arteries) were prospectively examined. All patients underwent intraarterial rotational angiography using a FPD system, and the cone-beam CT were reconstructed from the volume data set. Two radiologists evaluated the quality of the cone-beam CT, and then evaluated whether the additional information provided by the cone-beam CT was useful for the interventional procedures. In 41 (95\%) of 43 arteries, the extent of contrast material perfusion was sufficiently visualized on cone-beam CT. In 20 (47\%) of 43 arteries, the DSA plus cone-beam CT was superior to the DSA alone regarding the precise understanding of vascular territory of each artery. This information was helpful for predicting the drug delivery for superselective intraarterial chemotherapy, especially in a deeply invasive tumor with multiple feeding arteries. In the superselective intraarterial chemotherapy for head and neck tumors, the cone-beam CT with FPD provides the useful additional information, which allows interventional radiologists to determine the feeders as well as dose of antitumor agent for each feeder.

P4:97

\section{DIAGNOSTIC ACCURACY OF MRI FOR DETECTING ABNORMALITIES OF THE BRACHIAL PLEXUS IN PATIENTS WITH IDIOPATHIC BRACHIAL PLEXITIS}

S. KIM, J.H. Lee, H.K. Lim, J.H. Baek, S.J. Kim University of Ulsan College of Medicine, Asan Medical Center, Seoul, SOUTH KOREA 


\section{PURPOSE}

To evaluate the diagnostic accuracy of MRI for detecting abnormalities of the brachial plexus in patients with brachial plexitis.

METHODS

Among 74 patients with suspicious brachial plexopathy, fifteen patients finally diagnosed as brachial plexitis were enrolled in this retrospective study. All of them had unilateral involvement of the brachial plexus. Two neuroradiologists evaluated bilateral brachial plexus on MR images including coronal STIR, T2-, and pre/postenhanced T1-weighted images with consensus. The analysis was focused on the presence of hyperintensity on STIR or T2-weighted images, swelling, or enhancement of the individual components of the brachial plexus. We calculated the diagnostic accuracy of MRI by correlation with electrodiagnostic studies as a reference standard.

\section{RESULTS}

MR demonstrated abnormalities in 11 brachial plexuses of 15 patients with only 1 false positive result. The hyperintensity on STIR was the most common abnormality $(n=11)$, followed by the swelling $(n=3)$ and the enhancement $(n=2)$. All the true positive findings of the MR images were correlated well with the electrodiagnostic studies. From this results, the sensitivity, specificity, positive and negative predictive values and diagnostic accuracy of the MR images for the diagnosis of brachial plexitis were $66 \%, 93 \%, 91 \%, 74 \%$ and $80 \%$, respectively. CONCLUSION

MRI is helpful for detecting abnormalities of the brachial plexus in patients with brachial plexitis; hyperintensity on STIR images is the single most useful diagnostic criteria.

\section{P4:98}

\section{A CASE OF CARCINOSARCOMA EX PLEOMORPHIC ADENOMA ARISING IN THE SUBMANDIBULAR GLAND}

J. KIM, H. SEO, S. JEE

Seoul Veterans Hospital, Seoul, SOUTH KOREA

Carcinosarcoma which is composed of both carcinoma and sarcoma components, is an exceedingly rare tumor of the salivary gland. Sometimes it develops in the background of pleomorphic adenoma (PA) and is referred to as carcinoma ex PA.

In carcinoma ex PA, malignant transformation may follow stepwise sequence, and manifest as non-invasive carcinoma and then invasive carcinoma. The differential diagnosis is of clinical importance, because the prognosis of non-invasive carcinoma is excellent than that of invasive carcinoma. Here we present a unique case of carcinosarcoma ex PA in submandibular gland, that contains intraductal carcinoma and undifferentiated spindle cell sarcoma. Based on the clinical history and histopathology, we consider the lesion to have originated from recurrent PA. Here we present the computed tomography $(\mathrm{CT})$ imaging feature and its histopathologic appearance.

\section{P4:99}

\section{THE RADIOLOGICAL SPECTRUM OF COWDEN DISEASE}

\section{W. JUNG, H.J. PARK, J.H. BAEK, S.S. WHANG, J.Y. KIM}

Department of Diagnostic Radiology, St. Vincent's Hospital, College of Medicine, The Catholic University of Korea, Suwon, SOUTH KOREA

\section{PURPOSE}

To demonstrate the various imaging spectrums of Cowden disease. METHODS

Findings of imaging studies, including computed tomography (CT), ultrasonography and fluorodeoxyglucose positron emission tomography integrated with CT (FDG PET-CT), of a 50-year-old woman diagnosed with a history of breast cancer were reviewed. RESULTS

CT imaging showed multiple, variably low attenuation lesions of thyroid gland, lung, liver, bowel, muscle and other abdominal organs. Diffusely enlarged thyroid gland showed multiple fatcontaining nodules. Small hamartomatous lesions were seen in lung, intestine, pancreas, spleen and kidney. A large sized hemangioma was also seen in the liver. FDG PET-CT helped to differentiate multiple lesions from metastases.

\section{CONCLUSIONS}

In this presentation, our patient demonstrated the various imaging spectrums of Cowden disease, a rare autosomal dominant disease.

\section{P4:100}

\section{CT ANATOMY OF CONGENITAL CYSTS \\ OF THE HEAD AND NECK \\ WITH EMBRYOLOGICAL CORRELATION AND DISCUSSION OF DIFFERENTIAL DIAGNOSIS}

\section{J. JOHNSON $^{1}$, R. Carmody ${ }^{2}$, G.E. Green ${ }^{3}$, H. Burbank ${ }^{1}$}

${ }^{1}$ Fletcher Allen Health Care / University of Vermont, Department of Radiology, Burlington, VT, USA, ${ }^{2}$ University of Arizona, Department of Radiology, Tucson, AZ, USA, ${ }^{3}$ University of Michigan, Department of Pediatric Otolaryngology, Ann Arbor, MI, USA

\section{PURPOSE/AIM}

1. To review the normal CT anatomy of the branchial clefts and arches (BA) with embryological correlation.

2. To illustrate the imaging findings in classic congenital cystic masses affecting the head and neck.

3. To discuss the list of differential diagnosis when examining congenital cysts of the head and neck.

\section{METHODS}

The literature on the embryology and congenital cysts of the head and neck was reviewed. Appropriate illustrative cases from our respective institutions were analyzed. 3D models were reconstructed when necessary using OsiriX (Geneva, Switzerland) for educational purposes.

\section{RESULTS}

Radiographic anatomy of the branchial clefts and arches with focus on the maxilla, auricle, mandibular condyle, temporomandibular joint, thyroid, thymus gland, carotid artery and jugular veins was prepared. Embryological development of each structure is discussed. Examples prepared include branchial cleft cysts, thyroglossal duct cysts, ectopic thymus cysts, thyroid and parathyroid cysts, laryngoceles and ranulae, dermoid and teratoid cysts, and cystic vascular abnormalities such as the cystic lymphangioma. CONCLUSION

This exhibit reviews the normal anatomy and embryology of the branchial clefts and arches of the head and neck and correlates 
them with the spectrum of congenital cysts that occur in this region. After reviewing this exhibit, the reader will be familiar with the anatomy and congenital cysts of the head and neck and appropriate differential diagnoses.

\section{P4:101}

\section{DIAGNOSTIC VALUE OF VOLUME CT WITH RAY SUMMATION AND VOLUME RENDERING IN BONE FRACTURE OF THE HEAD}

\section{T. HOSOYA, A. ODA, M. KANOTO, Y. TOYOGUCHI}

Yamagata University - Department of Diagnostic Radiology, Yamagata, JAPAN

\section{PURPOSE}

To evaluate diagnostic value of volume CT in bonefracture of hte head compared with the skull X-ray films.

SUBJECTS AND METHODS

Fifteen patients ( 7 males and 8 females) with bone fractures of the head underwent both plain X-ray examination and brain CT with volume scan. From volume CT data, we made ray summation images using bone function with $0.3 \mathrm{~mm}$ reconstruction $(\mathrm{n}=7)$ or using soft tissue function with $1 \mathrm{~mm}$ reconstruction $(\mathrm{n}=8)$. All ray summation images were calculated in 2 separately parts (i.e. up and down, back and forth, and right and left). In 9 patients, volume rendering images were also obtained. We evaluated visually ray summation images and volume rendering images compared with the plain X-ray films.

\section{RESULTS AND DISCUSSION}

Ray summation images showed 10 fractures more clearly, 11 fractures nearly equal, and 3 fractures less clearly compared with the plain X-ray films. All 3 fractures obscured in ray summation images were obtained by soft tissue function with $1 \mathrm{~mm}$ reconstruction. Volume rendering images showed fractures more clearly or nearly equal to the plain X-ray films. Although CT has lower modulation transfer function (MTF) than the plain X-ray films, volume CT is superior to the plain X-ray films in diagnosis of bone fracture of the head. This may be because that ray summation images show less summation effect than the plain Xray films and volume rendering images have higher contrast resolution.

\section{CONCLUSION}

In diagnosis of bone fracture of the head, volume CT is superior or equal to plain X-ray films. In acute head trauma, plain X-ray films should be unnecessary.

\section{P4:102}

\section{IMAGING OF THE FIFTH CRANIAL NERVE: CROSS-SECTIONAL ANATOMY AND PATHOLOGY}

D. GARGAS, K. PALIALEXIS, D. MATSARIDIS, E. KOLOVOU, V. KOUTOULIDIS, A. KOUREAS, L.A. MOULOPOULOS, A. GOULIAMOS

UNIVERSITY OF ATHENS, ARETEION HOSPITAL, RADIOLOGY DEPARTMENT, ATHENS, GREECE

\section{PURPOSE}

To illustrate the normal course and pathology of the intracranial section of the fifth (trigeminal) cranial nerve using cross-sectional imaging.

METHODS

Thin section CT and MR images in various planes were obtained to optimally depict the intracranial course of the trigeminal nerve. Cases of trigeminal nerve lesions were collected.

RESULTS

The motor and sensory nerve roots that emerge from the lateral aspect of the midpons, the Gasserian ganglion on the apex of the petrous bone in the middle fossa and the three divisions of the nerve, each passing through its own foramen are depicted. Various pathologic conditions involving one or more trigeminal branches such as neurogenic tumors, leptomeningeal metastases and inflammatory processes are shown and discussed. CONCLUSIONS

The 5th cranial nerve is best demonstrated by MRI . Pathology of the trigeminal can be imaged effectively with the use of crosssectional imaging.

\section{P4:103}

\section{COLOR DOPPLER IMAGING OF THE OPHTHALMIC ARTERY IN PATIENTS WITH CHRONIC HEART FAILURE}

D. FREITAS, D. MEIRA-FREITAS, W. IARED, J. MELO-JR, A. PARANHOS-JR, D. ALMEIDA, S. ASJZEN UNIVERSIDADE FEDERAL DE SÃO PAULO (UNIFESP/ EPM), SÃO PAULO, BRAZIL

\section{PURPOSE}

Ocular blood flow is affected in several ocular pathological conditions, such as glaucoma. Despite this, the effects of reducing cardiac output on the perfusion of the optic nerve and its possible role in the pathogenesis of glaucoma in these patients are unknown. Then, we decided to evaluated alterations in color Doppler imaging of the ophtalmic artery in patietns with heart failure (HF).

METHODS

Doppler parameters of ophthalmic artery of 21 patients with heart failure were evaluated. These parameters were correlated with assessments by echocardiography, clinical status and changes of the optical disk. Finaly, they were compared with 21 controls without heart disease.

RESULTS

Nine men and 14 women with an average age of $50.7 \pm 11$ years, ranging from 27 to 72 years were studied. Gender and age of cases compared with controls were similar $(\mathrm{p}=0.9$ and $\mathrm{p}=1.0$, respectively). The average diastolic velocity of the cases was lower than that of controls, and this difference was significant $(p=0.01$ and $p=0.02$, right and left eyes, respectively). There was a correlation between the resistivity index of the ophthalmic artery and the diastolic blood pressure of patients with $\mathrm{HF}(\mathrm{p}=0.03$ and $\mathrm{p}=0009$, right and left eyes, respectively). The other studied parameters showed no statistical significance. There was low general correlation between the assessed Doppler parameters and changes in the optic disk. CONCLUSION

Reduced diastolic velocity in ophthalmic artery of patients with heart failure compared with controls was demonstrated. This 
finding, in correlation with literature data, may precede the glaucoma and be related to its vascular pathogenesis in these individuals. The dyastolic blood pressure appears to be a good clinical parameter in the determination of cardiac patients at higher risk of developing changes in ocular microvasculature.

\section{P4:104}

\section{CONGENITAL SENSORINEURAL HEARING LOSS: CORRELATION OF IMAGING FINDINGS AND ELECTRODIAGNOSTIC TESTING}

G. BEYER, S. GEBARSKI

University of Michigan, Ann Arbor, USA

\section{PURPOSE}

The purpose of this exhibit is to review normal inner ear anatomy, and discuss how thin-section CT, fast spin echo T2 MRI, and electrodiagnostic testing can be correlated to evaluate the cause of congenital sensorineural hearing loss in eight patients from our institution, as well as implications for treatment.

METHODS

Several cases of congenital sensorineural hearing loss diagnosed at our institution will be discussed. To begin, the normal inner ear anatomy and development will be reviewed, as well as common causes of inner ear pathology. The imaging techniques used as well as the findings in our patients will be detailed. These include thin-section CT and fast spin echo T2 MRI. The correlative results of imaging findings with electrodiagnostic testing for each case will be demonstrated. Finally, future directions and implications for treatment will be examined.

\section{CONCLUSIONS}

The causes of sensorineural hearing loss are varied and can occur along the pathway from cortex to the inner ear. Cochlear and facial nerve hypoplasia is one cause that often occurs in tandem with internal auditory canal dysplasia. CT, FSE T2 MRI, and electrodiagnostic testing each have diagnostic strengths and can be correlated to help determine the inner ear pathology. This helps to guide treatment and prognosis, particularly for cochlear implantation.

\section{P4:105}

\section{TUMORS AND TUMOR-LIKE LESIONS OF THE MANDIBLE AND MAXILLA: A PICTORIAL REVIEW}

\section{G. BEYER, H. PARMAR, M. IBRAHIM}

University of Michigan, Ann Arbor, MI, USA

\section{PURPOSE}

The purpose of this exhibit is to review the pathophysiology and imaging appearance of tumors and tumor-like lesions that commonly occur in the maxilla and mandibule using various imaging modalities including plain radiography, $\mathrm{CT}$, and MR imaging.

METHODS

This exhibit will review normal maxillofacial anatomy and development, and discuss pathophysiology of tumors and tumorlike lesions of the mandible and maxilla. The utility of various imaging modalities in the diagnostic workup of these lesions, as well as the imaging findings that can aid in narrowing the differential diagnosis will be discussed. Finally, the management implications will be reviewed.

CONCLUSIONS

Several tumors and tumor-like lesions specifically occur in the maxilla and mandible. The pathophysiology and appearance of these lesions on CT and MRI will be described including imaging findings that can aid in narrowing the differential diagnosis. The management implications will also be discussed.

\section{P4:106} CRANIAL NERVE V: A REVIEW OF ANATOMY,
AND PATHOLOGY OF THE TRIGEMINAL NERVE

\author{
G. BEYER, M. IBRAHIM, J. TROBE, H. PARMAR
}

University of Michigan, Ann Arbor, MI, USA

\section{PURPOSE}

The purpose of this exhibit is to review the anatomy and various pathology that can be found in the trigeminal nerve using examples from $\mathrm{CT}$ and MR imaging.

\section{METHODS}

Illustrations and cross sectional imaging (CT and MRI) examples of the anatomy and pathology of the four segments of the trigeminal nerve will be described and reviewed. This includes: the location of the nuclei,

the course of the cisternal segment, the location of Meckel's cave, and the V1-3 branches. Examples of pathology will include vascular lesions, infection, inflammatory processes such as multiple sclerosis, and masses.

\section{CONCLUSIONS}

The anatomy and function of the trigeminal nerve including segments will be reviewed. In addition, the clinical symptoms and imaging findings of pathology of CN V including vascular lesions, infection, inflammatory processes, and masses using CT and MRI will be discussed.

\section{P4:107}

\section{ENDOLYMPHATIC SAC TUMORS IN VON HIPPEL LINDAU DISEAE: MR IMAGING FINDINGS}

M. APAYDIN, M. APAYDIN, M. VARER, A. SARSILMAZ, G. COSKUN, E. ULUC, O. OYAR

IAEAH- Department of Radiology, IZMIR, TURKEY

Endolymphatic sac tumors are aggressive papillary tumors of the temporal bone frequently associated with von Hippel-Lindau disease (VHL). We present imaging findings of a VHL family with two cases of endolymphatic sac tumors.

They spread to the cerebellum and the cerebellopontine cistern, and may mimic other more frequent tumors of this region. These tumors shows predominantly high signal on T1 and T2-weighted images and they enhances heterogeneously.There are some difficulties in its differential diagnosis from other vascular and 
non-vascular tumors of the temporal bone. Increased signal intensity at unenhanced T1-weighted MR imaging is common and may help distinguish these lesions from more common, aggressive temporal bone tumors.

Endolymphatic sac tumors(ELSTs) are aggressive papillary tumors of the temporal bone frequently associated with von HippelLindau disease (VHL). These tumors are extremely rare in the general population. We present the imaging findings of two endolymphatic sac tumors with VHL.

\section{P4:108}

\section{KIMURA'S DISEASE: DIAGNOSTIC IMAGING WITH COMPUTED TOMOGRAPHY}

\section{A. ANIL GOPINATHAN, T.Y. TAN}

Department of Radiology, Changi General Hospital, Singapore, SINGAPORE

\section{AIM}

To establish a preoperative diagnostic system for Kimura's disease of the head and neck region using computed tomography.

METHODS

A retrospective study design was adopted. Thirteen patients with histopathological proven Kimura's disease had their clinical and pathological records reviewed besides a detailed analysis of their CT scans performed from base of skull to arch of aorta.

\section{RESULTS}

Both well defined nodular masses as well as ill-defined plaque like infiltrative masses were seen in the subcutaneous tissue of the head and neck region. All of them had lesions adjacent to the major salivary glands. Parotid gland was affected in 10 of the 13 cases and submandibular gland in the rest. Post contrast enhancement was variable. More than half of the cases had associated lymphadenopathy. Some of them showed atrophy of the skin and subcutaneous fat overlying the subcutaneous masses. Blood eosinophilia was a consistent feature in all the cases.

\section{CONCLUSION}

Computed tomography can be used as an effective modality in the non invasive diagnosis of Kimura's disease of the head and neck region.

\section{P4:109}

\section{WHERE DO THESE CYSTIC MASSES, FISTULAE AND SINUSES IN THE NECK COME FROM?}

\section{Y. Parag, K.A. Petropoulou, V. Agarwal}

Division of Neuroradiology, Department of Radiology - University of Pittsburgh Medical Center, Pittsburgh, PA, USA

\section{PURPOSE}

To review the embryology of the branchial apparatus and primitive pharynx and the derived developmental anomalies.

METHODS

CT and/or MRI appearance of the various developmental anomalies arising from the maldevelopment of the branchial apparatus and primitive pharynx will be discussed.

\section{RESULTS}

Some of the frequently encountered cystic masses in the neck in the pediatric population reflect maldevelopment of the branchial apparatus and primitive pharynx which form during early gestation and give rise to many structures in the neck.

The most common developmental anomalies are cysts of the second branchial cleft and thyroglossal duct. Cysts of the first, third or fourth branchial clefts, fistulae or sinuses deriving from the branchial apparatus in general as well as thymic cysts are far less common. It is noteworthy that these developmental anomalies often manifest themselves upon infection. Therefore knowledge of their imaging characteristics and frequent location are important as it may facilitate their diagnosis and impact patient's management.

CONCLUSIONS

1- Review the embryological development of the branchial apparatus and primitive pharynx.

2- Discuss the imaging characteristics of various cysts, fistulae and sinuses arising from their maldevelopment.

\section{P4:110}

\section{MESENHCHYMAL CHONDROSARCOMA OF CERVICAL AREA FROM C1 AND C2 AND SUBOCCIPITAL LEFT: CASE REPORT}

\author{
$\underline{\text { V. VUCKOVIC VASILJEVIC }}{ }^{1}$, D. Savic ${ }^{1}$, J. Sopta ${ }^{2}$, M. Skender \\ GazibaraInstitute $^{2}$ \\ ${ }^{1}$ Institute of Neurosurgery, Belgrad, SERBIA, ${ }^{2}$ Institute of \\ Pathology, Belgrad, SERBIA
}

\section{INTRODUCTION}

Mesenchymal chondrosarcoma (MS) is an extremely rare tumor, represented in less than $2 \%$ of all malign tumors of cartilage origin, of both skeletal or soft tissue presentation. We display a patient with mesenchymal chondrosarcoma which starts at suboccipital area and mastoid on the left side and is extended laterally on the left side of the neck forward up to interarticular space of $\mathrm{C} 1$ and $\mathrm{C} 2$ vertebrae.

MATERIALS AND METHOD

A patient, aged 63 is admitted to the Institute for Neurosurgery due to a huge tumor in the occipital area, left part and retroarticular. Before the admission to the hospital, a cranium base and neck CT was done, as well as a MR of endocranium. The MR and CT showed that the expansive formation is extracranial, starting from suboccipital area and mastoid on the left side and goes down on the left lateral side of the neck up to $\mathrm{C} 2$ vertebrae. The A selective panangiography of blood vessels was done, showing that the major part of tumor vascularisation comes from the left and vertebralis over the branch which mostly feeds the tumor itself.

It was decided to do firstly a tumor biopsy with removal of a part next to the occipital bone. A PH findings showed that it is a tumorous tissue of a similar aspect of chemangiopericitoma with a small focuses of hyaline - chondroide matrix.

Immunohystochemical tumor cells are CD34 I F-VIII negative, a focal CD99 I

S-100 are positive.

CONCLUSION

Malign and mesenchymal tumor of mesenchymal chondrosarcoma type. After this, it was decided to do a radical surgery intervention. 


\section{CONCLUSION}

Since mesenchymal chondrosarcoma (MS) is a rare tumor described as a separate identity 50 years ago and cases of this tumor are relatively rarely presented, for a final diagnosis it is necessary to have a PH i.e. immunohystochemical confirmation.

\section{Imaging in Epilepsy}

\section{P5:111}

\section{RADIOLOGIC FINDINGS IN PATIENT WITH MELAS PRESENTED WITH STATUS EPILEPTICUS}

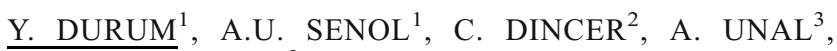
H. AYDIN GUNGOR ${ }^{3}$

${ }^{1}$ Akdeniz University Radiology Department, Antalya, TURKEY, ${ }^{2}$ Private Yasam Hospital Radiology Department, Antalya, TURKEY, ${ }^{3}$ Akdeniz University Neurology Department, Antalya, TURKEY

\section{PURPOSE}

We aimed to present radiologic findings in patient with MELAS presented with status epilepticus.

\section{MATERIAL-METHODS}

A 26-year-old women presented with status epilepticus. After initial neurologic evaluation, cranial CT and MRI were performed and the patient was admitted.

\section{RESULTS}

Contrast-enhanced cranial CT was performed in emergency department and there was no pathologic finding. On MRI, edematous changes and hyperintense areas on right temporo-parieto-occipital cortical region. DWI showed diffusion restriction in the same area. Findings were interpreted as postictal changes initially, but MELAS was too considered in differential diagnoses. Follow up was suggested. After four days, control MRI was performed because of unconsciousness. As well as the initial findings, hyperintensity and diffusion restriction were found in right thalamic area, along with slight shift of the midline structures. On MR spectroscopy, lactate peaks were present within the lesions. Because of progression in findings, muscle biopsy was performed and pathologic result was mitochondrial myopathy. Despite the supportive therapy, consciousness of the patient got worse. Control MRI was performed 17 days after the admission. T2 weighted MRI and DWI were revealed the same findings on the contralateral hemisphere.

CONCLUSION

MELAS should be considered in differential diagnosis in patients with diffuse cortical lesions. The progress of the involvement may be fast. Demonstration of lactate peaks is very helpful in making the diagnosis.

\section{P5:112}

\section{THE STUDY OF MOTOR ACTIVATION IN PATIENTS WITH CEREBELLAR ATROPHY (DUE TO LONG TERM ANTIEPILEPTIC DRUG MEDICATION) BY FMRI}

G. CHUNG, S. HWANG, Y. HAN, S. LEE

Chonbuk National University-Department of Radiology, Jeonju, SOUTH KOREA

\section{PURPOSE}

The aim of the study was to investigate the cortical activation of the primary motor cortex and cerebellum during both motor tasks in patients with cerebellar atrophy due to long term antiepileptic drug medication using fMRI.

MATERIALS AND METHODS

Fourteen patients with cerebellar atrophy due to long term epileptic medication and nine healthy subjects were examined. The activation tasks consisted of right and left finger complex movements. For each motor task, the thumb was apposed against each of the other fingers a different number of times. For statistical processing of the raw image data the SPM 5 software package was used. A p-value of $p<0.005$ was applied to differentiate between activated and non-activated. The resulting functional activation maps were superimposed onto the T1WI. The number of activated areas and contrast of parameter estimates were used to quantitate the cortical response upon motor activation.

\section{RESULTS}

The mean number of activated areas and contrast of parameter estimates were checked in left and right motor tasks. Healthy subjects showed 674 activated areas and 1.16 contrast of parameter estimates in right primary motor cortex, and 359, 0.58 in left cerebellum during left motor complex movement. While the patients with cerebellar atrophy showed 1987, 1.27 in right primary motor cortex and 219, 0.32 in left cerebellum during left motor complex movement.

During the right motor complex movement, healthy subjects showed 602, 1.04 in left primary motor cortex and 258, 0.52 in right cerebellum while patients with cerebellar atrophy showed $1332,1.32$ in left primary motor cortex and 140, 0.33 in right cerebellum.

\section{CONCLUSIONS}

In the patients with cerebellar atrophy, the activated areas and contrast of parameter estimates were larger in both side of motor cortex and lesser in cerebellum than those of the healthy subjects during motor tasks.

\section{P5:113}

\section{DOUBLE INVERSION RECOVERY IN PATIENTS WITH REFRACTORY EPILEPSY}

\author{
N. BARGALLÓ ${ }^{1}$, M. SQUARTIA ${ }^{2}$, X. SETOAIN ${ }^{2}$, M. CARREÑO $^{3}$, \\ A.J. DONAIRE $^{3}$, J. BERENGUER ${ }^{2}$, L. OLEAGA ${ }^{2}$ \\ ${ }^{1}$ CDIC. HOSPITAL CLINIC I PROVINCIAL DE BARCELONA. \\ IDIBAPS, BARCELONA, SPAIN, ${ }^{2}$ CDIC. HOSPITAL CLINIC I \\ PROVINCIAL DE BARCELONA, BARCELONA, SPAIN, \\ ${ }^{3}$ NEUROLOGY DEPARTMENT. HOSPITAL CLINIC I \\ PROVINCIAL DE BARCELONA, BARCELONA, SPAIN
}

\section{PURPOSE}

The purpose of this study was to identify the role of DIR (double inversion recovery) sequence, that has been suggested to improve the sensitivity in finding lesions with low T2 contrast in the identification of lesions in patients with refractory epilepsy.

MATERIAL AND METHODS

Forty patients with refractory epilepsy have been studied in a $3 \mathrm{~T}$ scan, using a specific epilepsy protocol that also includes a $3 \mathrm{D}$ 
coronal DIR ( $1 \mathrm{~mm}$ slice, matrix $192 \times 192$ and FOV $225 \times 199$ ). Detection of lesions, suspected etiologic and evaluation of which sequence was better to characterize the lesion were done using visual inspection by an experienced neuroradiologist.

RESULTS

Only 25 patients have shown lesions in almost one of the three sequences. ( 14 patients with mesial sclerosis, six of them with dual pathology; 7 patients with malformation of cortical development, three of the with multiple displasias ; 3 tumours, two of them were cavernomas and one patient has posttraumatic malacic changes. Overall sensitivity was similar between FLAIR and DIR, although DIR was slightly higher. DIR sequence was better than FLAIR in $52 \%$, equal in $16 \%$, and worst in $8 \%$ of the cases; DIR was better than T2 in $48 \%$, equal in $32 \%$ and worst in $16 \%$. In two patients DIR was the only sequence that showed abnormalities (hippocampus and amygdale lesions). However patients with calcification or hemosiderin were better characterized with $\mathrm{T} 2$ sequences.

\section{CONCLUSION}

DIR is a sequence providing high sensitivity to detect lesions in patients with refractory epilepsy. Although its sensibility is similar to FLAIR, DIR might be useful as an additional sequence when other conventional sequences are negative or reveal subtle or unclear lesions.

\section{P5:114}

\section{DETECTING HIPPOCAMPAL SCLEROSIS (HS) WITH 1H-MAGNETIC RESONANCE SPECTROSCOPY (MRS)}

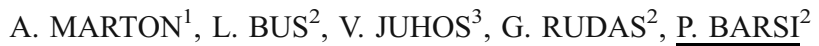 \\ ${ }^{1}$ Semmlweis University, Faculty of Medicine, Budapest, HUNGARY, \\ ${ }^{2}$ Semmelweis University, MR Research Centre, Budapest, \\ HUNGARY, ${ }^{3}$ St. István's Hospital, Dept. of Neurology, Budapest, \\ HUNGARY
}

\section{PURPOSE}

To develop an MRS method that reliably detects metabolic alterations in HS and can function later as a complement to the evaluation of temporal lobe epilepsy (TLE).

\section{METHODS}

18 patients (mean age $41+/-10$ yrs) with TLE were studied by 3 T MR. Previous MRI using epilepsy protocol showed 13 unilateral HS and 5 without HS. MRS was performed using PRESS spin echo sequence set with 128 averages. Repetition time was $2000 \mathrm{~ms}$, short (TE1 $35 \mathrm{~ms}$ ) and long echo times (TE2 $288 \mathrm{~ms})$ were used. The volume of interest (VOI) $(30 \times$ $8 \times 6 \mathrm{~mm}, 1440 \mathrm{~mm} 3)$ was tilted to the hippocampus. N-acetylaspartate (NAA)/choline (Cho), NAA/creatin $(\mathrm{Cr})$ and $\mathrm{Cho} / \mathrm{Cr}$ ratios were calculated from peak area measurements. Lateralization was determined by using asymmetry coefficient (Ca). Results were compared with MRI as standard reference and with EEG.

\section{RESULTS}

Mean NAA/Cho and NAA/Cr were lower, mean $\mathrm{Cho/Cr}$ was higher on the affected side. The most consistent MRS parameter for lateralization was $\mathrm{Ca}$ for NAA/Cho with a sensitivity of $75 \%$ with TE1 and $67 \%$ with TE2. The correct lateralization rates were $67 \%$ (TE1) or $50 \%$ (TE2) and $84 \%$ or $58 \%$ as for $\mathrm{NAA} / \mathrm{Cr}$ and $\mathrm{Cho} / \mathrm{Cr}$ respectively. Mean $\mathrm{Ca}$ in unilateral cases was higher consistently from those with no HS, but was significant only for $\mathrm{Cho} / \mathrm{Cr}(\mathrm{p} 0,021)$ with $\mathrm{TE} 1$ and $\mathrm{NAA} / \mathrm{Cr}(\mathrm{p} 0,049)$ with TE2. The concordance rates of lateralization with EEG and MRS was $50-84 \%$, depending on which ratio was used.

\section{CONCLUSIONS}

Our data provided readable curves in reasonable time, in accordance with the known pathophysiology of HS. Our results show that using a small, but elongated VOI (to include most of the hippocampus while excluding surrounding structures) and using $\mathrm{Ca}$ for detecting the presence of abnormality are preferable. Larger sample populations to reach statistical significance and involving healthy volunteers for the identification of bilateral alterations are needed.

\section{P5:115}

\section{MR CHARACTERISTIC OF FOCAL CORTICAL DYSPLASIA}

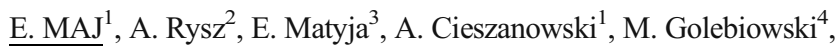
A. Marchel $^{2}$, O. Rowinski ${ }^{1}$

${ }^{1}$ 2-nd Department of Clinical Radiology, Medical University of Warsaw, Warsaw, POLAND, ${ }^{2}$ Department of Neurosurgery, Medical University of Warsaw, Warsaw, POLAND, ${ }^{3}$ Department of Experimental and Clinical Neuropathology, M. Mossakowski Medical Research Centre, Polish Academy of Scienc, Warsaw, POLAND, ${ }^{4} 1$ st Department of Clinical Radiology, Medical University of Warsaw, Warsaw, POLAND

\section{PURPOSE}

Focal cortical dysplasia (FCD) represents abnormal neuronal migration, proliferation and differentiation and is one of the most common causes of drug-resistant epilepsy, often affecting children and young adults. Four types of FCD are recognised: IA, IB, 2A, 2B (Taylor type). The aim of this study was to identify and differentiate types of FDC in MR imaging.

\section{METHODS}

Retrospective analysis of MR studies of 42 patients, operated due to drug-resistant epilepsy, with histological confirmation of FDC alone or FDC coexisting with hippocampal sclerosis, was performed. Studies were performed on $1.5 \mathrm{~T}$ scanner using sequences: T1SE (axial, saggital), T2TSE, FLAIR (axial, coronal), IR (coronal). The study group consisted of 42 patients: 25 women and 17 men (age range: 17-55 years; mean-32).

\section{RESULTS}

The most common type of FDC was 2A (25 patients), type 1A was identified in 9 patients, type $1 \mathrm{~B}$ in 5 , type $2 \mathrm{~B}$ in 2 and in one coexistence of types $2 \mathrm{~A}$ and $2 \mathrm{~B}$ was noted. Hippocampal sclerosis coexisted in 19 cases with FDC: with type $2 \mathrm{~A}$ in 14 , type $1 \mathrm{~A}$ in 3 and type $1 \mathrm{~B}$ in 2 patients. Abnormalities were identified on MRI in 35 patients (83\%): 21 in type $2 \mathrm{~A}(50 \%)$, 8 in type $1 \mathrm{~A}(19 \%), 4$ in type $1 \mathrm{~B}(9,5 \%), 2$ in type $2 \mathrm{~B}(4,8 \%)$. 


\section{CONCLUSIONS}

MRI is useful in detection of causes of drug-resistant epilepsy. It demonstrates high efficiency in diagnosis of FDC and hippocampal sclerosis.

\section{P5:116}

\section{CLONAZEPAM CAN ASSIST PERFORMING SENSORIMOTOR FUNCTIONAL MRI EXAMINATIONS IN STATUS EPILEPTICUS DURING SLEEP}

L. KOZAK $^{1}$, M. Hegyi ${ }^{2}$, P. Barsi ${ }^{1}$, G. Rudas ${ }^{1}$

${ }^{1}$ Semmelweis University-MR Research Center, Budapest, HUNGARY, ${ }^{2}$ Bethesda Children's Hospital of Hungarian Reformed Church-Department of Neurology, Budapest, HUNGARY

\section{INTRODUCTION}

Functional magnetic resonance imaging (fMRI) examinations became an integral part of epilepsy surgery workup. In the pediatric population these examinations are usually carried out in full anesthesia, however in some forms of epilepsy, e.g. electrical status epilepticus in sleep (ESES), anesthesia could jeopardize the success of the examination.

METHODS

We have performed functional MRI (fMRI) in two sessions for the identification of sensory-motor areas in an eight-year-old polymicrogyric child, having continuous generalized spike-and-wave activity during sleep. The examination protocol included a high resolution $3 \mathrm{D} \mathrm{T} 1 \mathrm{~W}$ sequence for anatomy and two $\mathrm{T} 2 * \mathrm{~W}$ EPI sequences for fMRI acquisition. The fMRI paradigm consisted of alternating blocks of passive wrist movement and rest. Both sessions were performed in propofol induced general anesthesia with co-administration of clonazepam in the second session. EEG data were also collected in both sessions before the MR acquisition.

\section{RESULTS}

Passive wrist movements utilizing propofol anesthesia alone provided no valuable information, however with the coadministration of clonazepam we could observe clear and significant sensorimotor activations showing clear-cut functional reorganization of sensory-motor areas towards the unaffected side of the brain. EEG measurements showed generalized spike-andwave activity in anesthesia, which disappeared in the second session after the administration of clonazepam.

\section{CONCLUSIONS}

The inefficiency of the first fMRI session might stem from the hemodynamic "noise" caused by uncoordinated neural responses during the generalized spike-and-wave activity that masks the stimulus-event related BOLD response. Since spike-and-wave activity, and thus the hemodynamic "noise" disappeared in the second session, we conclude that clonazepam has a favorable effect for performing fMRI in cases of ESES, and possibly in other epilepsies, as well. Furthermore, among the methodological issues addressed, this case provides evidence for a post-hemispherotomy like functional redistribution of sensorimotor activations to the unaffected hemisphere in a case of polymicrogyria-related childhood epilepsy.
Imaging of Brain Tumors-Multimodality Approach

\section{P6:117}

\section{UNUSUAL MENINGEAL MASSES: MR AND PATHOLOGIC CORRELATION AND REVIEW OF THE LITERATURE}

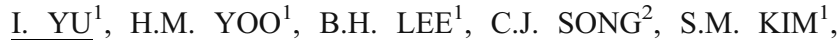 \\ H.K. KIM ${ }^{1}$ \\ ${ }^{1}$ EULJI UNIVERSITY HOSPITAL, DAEJEON, SOUTH \\ KOREA, ${ }^{2}$ CHUNGNAM UNIVERSITY HOSPITAL, DAEJEON, \\ SOUTH KOREA
}

\section{PURPOSE}

Sometimes there are intraaxial-looking masses, which are turned out to be meningeal mass, where as there area conventional meningima-looking mass, which are turned out to be unusual meningeal lesions.

This poster presents the MR and pathological features of these intraaxial-looking meningeal masses and conventional meningiomalooking unusual meningeal lesions and review the literature.

METHODS

We reviewed the clinical, MR and pathological features of intraaaxial-looking meningeal masses, including angiomatous meningioma, microcytic meningioma, atypical meningiomas, chordoid meningioma, clear cell meningioma, rhabdoid meningioma, anaplastic meningioma, hemangiopericytoma and meningioma-looking unusual meningeal lesions including dural lymphoma, idiopathic hypertrophic pachymeningitis and meningeal melanosis.

RESULTS

Unusual meningeal masses such as angiomatous, microcytic, atypical, chordoid, clear cell, anaplastic, rhabdoid meningiomas and hemangiopericytomas were often misdiagnosed as intraaxial malignant masses because of relatively narrow attachement to the dura, or poor enhancement, or lobulating irregular outer margin, while other types of unusual meningeal masses such as dural lymphoma, idiopathic hypertrophic pachymeningitis and meningeal melanosis were often thought as conventional meningiomas or even subdural or subarachnoid hematoma because of their various mimicking features. CONCLUSION

Unusual meningeal masses are often misdiagnosed as being malignant intraaxial masses or conventional meningioma or even extracerebral hematoma because of their various mimicking features. To understand the clinical, MR and pathological features of them help the accurate diagnosis and the appropriate treatment.

\section{P6:118}

\section{UNUSUAL RARE PITUITARY LESIONS MASQUERING PITUITARY ADENOMA: MR AND PATHOLOGIC FEATURES} AND REVIEW OF THE LITERATURE

\author{
I. YU ${ }^{1}$, H.M. Yoo ${ }^{1}$, B.H. Lee ${ }^{1}$, C.J. Song ${ }^{2}$, S.M. Kim ${ }^{1}$, H.K. Kim ${ }^{1}$ \\ ${ }^{1}$ Eulji university hospital, Daejeon, SOUTH KOREA, ${ }^{2}$ Chungnam \\ university hospital, Daejeon, SOUTH KOREA
}




\section{PURPOSE}

Sometimes, there are unusual rare entity pituitary lesions, which are preoperatively diagnosed as pituitary adenoma. This poster presents these unusual rare pituitary lesions by demonstrating MR and pathologic features and reviewing the literature.

\section{METHODS}

We reviewed the clinical, MR and pathological features of unusual rare pituitary lesions masquering pituitary adenoma, including inflammatory pituitary pseudotumor, invasive fungal infection, lymphocytic hypophysiitis, pituitary abscess, pituitary lymphoma and Rathke's cleft cyst.

\section{RESULTS}

Inflammatory pituitary pseudotumor showed diffuse dark T2 SI with mild homogeneous enhancement and invasion to both cavernous sinus, masquering invasive pituitary adenoma. Lymphocytic hypophysiitis showed sellar enlargement of heterogeneous SI, homogeneous enhancement, mimicking pituitary adenoma, but with disappearing hyperintense SI of neurophypophysis. Pituitary abscess showed sellar enlargement of heterogeneous low T1 SI, high T2 SI with peripheral rim enhancement, mimicking cystic pituitary adenoma. Pituitary lymphoma showed homogeneously enhancing mass involving both pituitary gland and pituitary stalk. Rathke's cleft cyst showed intrasellar cystic mass centered in the pars intermedia, usually containing dark T2 SI debris in the dependent portion of the cystic mass.

\section{CONCLUSION}

There are sometimes unusual rare pituitary lesions masquering pituitary adenoma because of their various mimicking features. To understand the clinical, MR and pathological features of them help the accurate diagnosis and the appropriate treatment.

\section{P6:119}

\section{INTRACANIAL LYMPHOMAS. A PICTORIAL REVIEW}

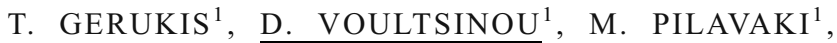
K. ANASTASIADOU ${ }^{1}$, V. KALPAKIDIS ${ }^{1}$, P. PALLADAS ${ }^{1}$, P. PRASOPOULOS ${ }^{2}$

${ }^{1}$ Department of Radiology of G. Papanikolaou Hospital of Thessaloniki, THESSALONIKI, GREECE, ${ }^{2}$ Department of Radiology, University Hospital of Alexandroupolis., ALEXANDROUPOLIS, GREECE

\section{PURPOSE}

To review the MRI appearances of primary and secondary brain lymphomas and its complications.

\section{METHODS}

Eleven patients with intracranial lymphoma or lymphoma complications were evaluated by conventional MRI as well as by Diffusion Weighted imaging(DWI), perfusion weighted imaging (PWI). Primary lymphoma was observed in 6 immunocompetent (IC) (1 patient with intravascular lymphomatosis) and 1 immunocompromized patients(ICM). Secondary brain lymphoma was demonstrated in 3 patients, 2 with systemic and 1 with gastric non-Hodgkin. Brain abscess developed in 2 patients with systemic non-Hodgkin lymphoma.

\section{RESULTS}

MRI in primary brain lymphoma of (IC) revealed solitary lesions that were located in the frontal and parietal lobe of subependymal regions and deep gray matter with nearly homogeneous contrast enhancement. Intravascular lymphomatosis demonstrated multiple lesions diffusely distributed along the perivascular spaces with dot like enhancement. In ICM patient, multiple lesions were observed in same areas as in IC having analogous pattern of contrast enhancement. In secondary lymphomas MRI examination showed cerebellar $(n=2 / 3)$ and supratentorial $(n=1 / 3)$ solitary lesion with homogeneous enhancement. The abscesses were demonstrated supratentorial location. DWI demonstrated restricted diffusion at the tumor, with mean $\mathrm{ADC}$ values 1.35 with +0.11 . PWI at the tumor depicted $\mathrm{CBV}$ with mean values $2.63+-1.48$. Significant degree of restriction observed in abscesses with mean ADC values 0.41 with +0.12 .

\section{CONCLUSIONS}

Intracranial lymphoma presents as solitary (in immunocompetent patients and secondary lymphoma) or multiple lesions (in immunocompomised patients and intravascular lymphomatosis). Primary intracranial lymphoma showed supratentorial location mainly at the frontal and parietal lobe either subependymal or at subcortical and deep gray matter.

Secondary lymphoma observed at both supratentorial and infratentorial location. Both primary and secondary lymphoma revealed homogeneous enhancement with exception intravascular lymphomatosis which demonstrated dot like enhancement. All the lesions presented with restricted diffusion, low ADC and perfusion values.

\section{P6:120}

\section{DIFFUSION WEIGHTED MR IMAGING OF BRAIN TUMORS} T. GERUKIS, D. VOULTSINOU, A. PETRIDIS, E. VAFIADIS,
V. KALPAKIDIS, P. PALLADAS

Department of Radiology of G. Papanikolaou Hospital of Thessaloniki, THESSALONIKI, GREECE

\section{PURPOSE}

To evaluate the DWI-MR imaging in tumor grading, in distinguishing between brain abscess and necrotic cystic neoplasm, high grade gliomas and lymphomas-leukemic involvement as well as arachnoids cysts and epidermoid tumor.

METHODS

We evaluated a total of $n=79$ patients, with high grade gliomas $n=18$, with low grade glioma $n=8$, with gliomatosis cerebri $n=4$, with brain metastases $n=21$, with lymphoma $n=10$, with leukemic involvement $\mathrm{n}=3$, with brain abscess $\mathrm{n}=5$, with arachnoid cyst $\mathrm{n}=4$ and with epidermoid tumor $n=1$, with conventional MR Imaging as well as diffusion weighted imaging DWI with ADC mapping.

RESULTS

Higher ADC values were observed in lower grade primary brain tumors and lower in high grade tumors. Brain abscesses revealed increased signal intensity on DWI and decreased signal intensity on ADC map images compared to cystic-necrotic neoplasms that showed deceased signal intensity on DWI and 4 to 10 times increased ADC values. A significant overlap in ADC values was 
observed between metastases, gliomas and lymphomas. Arachnoid cysts were hypointense in DWI with ADC values similar to subarachoid fluid in contrast with epidemoid cyst that appeared hyperintense in DWI with similar ADC values to those of the brain parenchyma.

The diagnosis was confirmed with biopsy (stereotactic and after surgery) in $n=53$ patients and serial follow up examinations in $n=$ 26 patients.

\section{CONCLUSIONS}

DWI is an important aid in tumor grading, in distinguishing between abscess and necrotic neoplasm, arachnoid cyst and epidermoid tumor but possesses important limitation in distinction between high grade tumors, solitary metastasis, lymphoma and leukemic involvement.

\section{P6:121}

\section{DIFFUSION AND PERFUSION WEIGHTED MAGNETIC RESONANCE IMAGING IN DISTINCTION BETWEEN SOLITARY BRAIN METASTASES AND HIGH GRADE GLIOMAS}

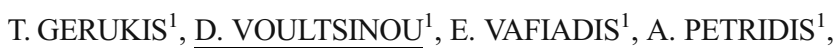
V. KALPAKIDIS ${ }^{1}$, P. PALLADAS ${ }^{1}$, P. PRASOPOULOS ${ }^{2}$

${ }^{1}$ Department of radiology, G. Papanikolaou Hospital of Thessaloniki, THESSALONIKI, GREECE, ${ }^{2}$ Department of Radiology, University Hospital of Alexandroupolis, ALEXANDROUPOLIS, GREECE

\section{PURPOSE}

To examine the feasibility of diffusion weighted imaging (DWI) and perfusion weighted imaging (PWI) in differentiating solitary brain metastases (SBM) from high grade gliomas (HGG).

METHODS

The study includes 39 patients with solitary brain lesion, solitary metastases $(n=21)$ or high grade (IV grade) gliomas $(n=18)$ histologically confirmed. These entities may have similar appearance on conversional MR sequences.

All patients underwent single shot echo planar DWI with $3 \mathrm{~b}$ values $(0,500,1000 \mathrm{~s} / \mathrm{mm} 2)$. Apparent diffusion coefficient (ADC) and cerebral blood volume (CBV) maps were generated. The CBV and ADC maps of the lesion were referred to the FLAIR and contrastenhanced T1 images at the same levels to determine the boundaries of the lesions (enhancing portion) and the extent of peritumoral edema. The paired Student $t$ test and Mann Whitney were used for statistical analysis.

\section{RESULTS}

The ADC values at the tumor and peritumoral area were $1.30+-0.29$ and $1.30+-0.26$ respectively, for SBM and $1.20+-0.32$ and $1.35+$ -0.26 respectively, for $\mathrm{HGG}$. There were no statistical difference in ADC values ( $p$ greater or equal to 0.97 ) between SBM and HGG. The CBV values in the immediate peritumoral region were $12.30+-9.35$ and $2.39+-1.31$ respectively, for metastasis and $13.81+-9.21$ and $6.39+-4.39$ respectively, for gliomas. CBV values for peritumoral area differ $(\mathrm{p}=0.04)$ between SBM and HGG while no difference $(\mathrm{p}=$ 0.98) was observed in CBV values at the tumor area.

\section{CONCLUSIONS}

$\mathrm{CBV}$ values for peritumoral region may assist the distinction between SBM and HGG. These difference in CBV values could be related with the presence of peritumoral neoplastic cell infiltration in HGG. ADC values were not found of any vale in discriminating SBM from $\mathrm{HHG}$.

\section{P6:122}

\section{DTI AND 1H-MRS EVALUATION IN DIFFERENTIATING THE CEREBRAL TUMORS}

C. SZARY ${ }^{1}$, J. Walecki $^{2}$, T. Bulski ${ }^{2}$, R. Boguslawska-Walecka ${ }^{3}$, G. Zielinski ${ }^{3}$

${ }^{1}$ Department of Radiology and Diagnostic Imaging, Hospital Ministry of Internal Affairs Administration, Woloska 137, Warsaw, POLAND, ${ }^{2}$ Department of Radiology and Diagnostic Imaging, Medical Centre for Postgraduate Education, Warsaw, POLAND, ${ }^{3}$ Department of Radiology and Diagnostic Imaging, Military Institute of Health Service, Warsaw, POLAND

\section{PURPOSE}

The diagnostic potential of new combined functional neuroimaging modalities like magnetic resonance spectroscopy (MRS) and quantitative diffusion tensor analysis - fractional anisotropy (FA) and measurement of apparent diffusion coefficient (ADC)-were investigated in relation with conventional MR imaging in presurgical period. These methods were used in differentiating the most frequent types of brain tumors (gliomas, meningiomas and solitary brain metastases).

METHODS

Multimodal magnetic resonance assessment of brain tumors (conventional MR, MRS, quantitative diffusion tensor and ADC analysis) were performed in 32 patients. Preoperative radiological results were followed by cerebral biopsy to prove the value of new diagnostic approach.

RESULTS

Preliminary results confirmed by histopathological cerebral biopsy of brain tumors demonstrate that this multimodal and combined new diagnostic approach can be sufficient in differentiating among the most common brain neoplasms.

CONCLUSIONS

Measurement of ADC and FA values in the intra-and peritumoral areas and comparing them with conventional magnetic resonance and MRS let us differentiate the type of brain tumor and determine the spatial distributions of neoplastic tissue (peritumoral vasogenic edema, infiltration with or without local destruction of white matter bundles). This combined diagnostic approach improves neurosurgical planning and postoperative results.

\section{P6:123}

\section{MRI OF GLIOBLASTOMA MULTIFORM. IDENTIFICATION WITH BRAIN DIFFUSION WEIGHTED MR IMAGING}

E. Testempasi ${ }^{1}$, V. Ouranos ${ }^{1}$, K. Stefanidis ${ }^{1}$, C. Louizakis ${ }^{2}$, I. Skouras $^{1}$, E. Kratimenou ${ }^{1}$, S. Benakis ${ }^{1}$, T. Vagdatlis ${ }^{1}$, D. Chondros ${ }^{1}$ ${ }^{1}$ Evangelismos Hospital, CT \& MRI Department, Athens, GREECE, ${ }^{2}$ Polikliniki Hospital, Department of Radiology, Athens, GREECE 


\section{PURPOSE}

The aim of this study is to describe the MR features of Glioblastoma Multiform, investigate them with diffusion-weighted imaging (DWI) and calculate the apparent diffusion coefficient values (ADC).

\section{METHODS}

We retrospectively analyzed 14 patients (range $26-58$ y.o.), with histologically proven glioblastomas, seen in our department during the last year. In addition to conventional sequences of routine tumor protocol, the cystic-necrotic components and the solid parts of the tumors, were evaluated on the DWI and the apparent diffusion coefficient (ADC) values were calculated.

RESULTS

11 patients showed an heterogeneous mass with cystic-necrotic components of variable size and extension, and a patchy, nodular or ring enhancement. In 3 patients multiple lesions were depicted. All tumors exhibited hypointensity on T1 sequences and areas of high signal intensity on T2, PD and flair sequences with adjacent surrounding edema. In 3 patients, solid or patchy enhancing lesions without central necrosis were present. In 5 cases hemorrhage was seen within the mass or the tumor margins. DWI showed areas of increased signal intensity in all cases. ADC values obtained in solid-enhancing areas of the lesions ranged from $0,79-1,261 \times 10^{-3} \mathrm{~mm}^{2} / \mathrm{s}$, (mean value $1.03 \pm 0,210$ ). In 12 patients the cystic-necrotic component of the lesions, demonstrated low signal intensity in DWI and the ADC values varied between $1.65 \times 10^{-3} \mathrm{~mm}^{2} / \mathrm{s}$ and $3.1 \times 10^{-3} \mathrm{~mm}^{2} / \mathrm{s}$ (mean value $2.276 \pm 0.41 \times 10^{-3} \mathrm{~mm}^{2} / \mathrm{s}$ ). In two of the cases apparent high signal intensity in DWI was interpreted and the ADC values varied between $0.426 \times 10^{-3} \mathrm{~mm}^{2} / \mathrm{s}$ and $1.19 \times 10^{-3} \mathrm{~mm}^{2} / \mathrm{s}$.

\section{CONCLUSIONS}

In most cases of glioblastomas, cystic components do not exhibit restricted diffusion in DWI, but some of them display homogeneous or heterogeneous high signal intensity and decrease of ADC values. DWI MRI imaging could be helpful in the differential diagnosis of these tumors.

\section{P6:124}

\section{CT PERFUSION IMAGING IN DETECTION OF SMALL RECURRENT OR RESIDUAL JUVENILE NASOPHARYNGEAL ANGIOFIBROMAS}

I. SHCHUROVA, M. Nersesyan, I. Pronin, V. Kornienko, D. Kapitanov, L. Fadeeva

Burdenko Neurosurgical Institute-Neuroimaging department, Moscow, RUSSIA

\section{INTRODUCTION}

Juvenile nasopharyngeal angiofibroma (JNA) is a highly vascular, locally invasive tumor with a high incidence of persistence and recurrence.

\section{OBJECTIVE}

To evaluate CT perfusion due to detecting residual and recurrent JNA.

\section{MATERIALS AND METHODS}

Fourteen patients with JNA were analyzed before and after endoscopic removal of JNA with CT perfusion study. The cerebral blood flow (CBF), cerebral blood volume (CBV) and mean transit time (MTT) were measured in the nasal mucosa, temporal muscle, the normal areas of brain and in JNA.

RESULTS

CBV, CBF of JNA were significantly greater in all fourteen patients comparably with nasal mucosa, temporal muscle and normal brain preoperatively. Postoperatively $\mathrm{CBV}, \mathrm{CBF}$ of the nasal mucosa were higher then of brain tissue and temporal muscle but still lower then $\mathrm{CBV}$ and $\mathrm{CBF}$ of residual JNA in all patients, with statistically significant $p$ values. MTT of JNA had greater prolongation then MTT of the brain in 10 patients, while identical in four patients; it was lower then temporal muscle in all patients and there was no significant difference MTT between JNA and nasal mucosa. Significant differences of hemodynamic characteristics helped to differentiate clearly JNA from nasal mucosa, temporal muscle and brain tissue.

\section{CONCLUSION}

CT perfusion is a new minimally invasive method of diagnostic JNA. Its more important application is to detect even small recurrent or residual JNA postoperatively.

\section{P6:125}

\section{SURVEY OF TWO PATIENTS WITH GLIOMATOSIS CEREBRI (GC) WITH NON-CONVENTIONAL MAGNETIC RESONANCE IMAGING (MRI )TECHNIQUES}

K. TSEKOURAS ${ }^{1}$, E. PAPADAKI ${ }^{1}$, V. MASTORODIMOS ${ }^{2}$, V. STILIANAKI ${ }^{1}$, E. AMANAKIS ${ }^{1}$, T. MARIS $^{3}$, S. KARAMPEKIOS ${ }^{1}$ ${ }^{1}$ UNIVERSITY HOSPITAL, DEPARTMENT OF RADIOLOGY, HERAKLION, GREECE, ${ }^{2}$ UNIVERSITY HOSPITAL, DEPARTMENT OF NEUROLOGY, HERAKLION, GREECE, ${ }^{3}$ UNIVERSITY HOSPITAL, DEPARTMENT OF MEDICAL PHYSICS, HERAKLION, GREECE

\section{PURPOSE}

Gliomatosis Cerebri (GC) is a rare malignant neuroepithelial tumor of uncertain origin that affects all ages of both sexes. We present two cases of GC and discuss the role of conventional and non-conventional MRI techniques, such as Diffusion Weighted Imaging (DWI), Perfusion Weighted Imaging (PWI) and Magnetic Resonance Spectroscopy (MRS), in the diagnosis and management of GC.

\section{MATERIAL AND METHODS}

A 18 year old male (pt1) and a 55 year old female (pt2) with clear personal and family history presented with intermittent memory disorders and disorientation. Both patients were studied at a $1.5 \mathrm{~T}$ MR scanner and non-conventional MRI techniques were performed : DWI (T2 SE EPI using 6 gradient fields), PWI (dynamic T2*W GRE EPI during the first pass of a standard dose of $0.1 \mathrm{mmol} / \mathrm{kgr}$ bolus of gadopentate dimeglumine) and MRS ( PRESS technique with single-voxel acquisition and short $\mathrm{TE}=35 \mathrm{~ms})$.

RESULTS

In both patients the conventional MRI revealed a process occupying both thalami, basal ganglia, most of the brainstem 
and part of the medial temporal lobes (in patient 2), that was hyperintense on T2W and FLAIR images, hypointense on T1W images, while after intravenous injection of paramagnetic contrast medium no enhancement was noticed. DWI revealed restricted diffusion at the affected areas, while the mean $\mathrm{rCBV}$ (relative cerebral blood volume) values were not elevated. MRS technique showed marked increase of myoinositol ( $\mathrm{mI})$ and creatine $(\mathrm{Cr})$ and normal levels of choline (Cho). Stereotactic biopsy was performed in pt 2 with findings consistent with a diffusely infiltrating malignancy of astrocyte origin, diagnostic for GC. Both patients passed away a few months after their first presentation.

\section{CONCLUSION}

Patients with GC have characteristic imaging findings in the nonconventional MRI techniques, quite different from other primary brain tumors, that could be almost pathognomonic for the disease.

P6:126

\section{THE ROLE OF PET IN THE DIFFERENTIATION OF TUMOR EDEMA OR RECURRENCE AFTER X-KNIFE STEREOTACTIC RADIOSURGERY}

C. CHRISSICOPOUlOS, S. Mourgela, K. Kirgiannis, K. Petritsis, A. Spanos

Agios Savvas Anticancer Institute, Athens, GREECE

\section{PURPOSE}

Metastatic brain tumors occur more frequently than primary brain tumors. Patients with one or more than one metastatic tumor, that is not readily surgically accessible, are typically treated with radiation therapy. Recently stereotactic radiosurgery, which is a more targeted radiation therapy, and is not actually surgery at all, is used to treat solitary deep situated brain tumors. Possible side effects of radiosurgery include swelling which must be differentiated from recurrence. We present such a case in order to delineate the role of PET imaging in the differential diagnosis of such cases.

\section{CASE PRESENTATION}

A 43-year old woman with a history of breast carcinoma, presented three years ago with a 2 months history of dizziness and headache, which progressively worsened. Brain MRI revealed a tumor in the right temporo-occipital area $2,5 \times 2 \mathrm{~cm}$ in size, which was a metastasis (Fig. 1). Debulking of the tumor by stereotactic surgery (X-knife) was subsequently performed (dose 20-28,8 Gy). Six months after stereotactic radiotherapy an augmentation of the tumor size was noted, which could represent either tumor edema or recurrence (Fig. 2). PET scan was performed, which showed no evidence of tumor recurrence (Fig.3). Follow-up MRI examination one year later revealed reduction of the tumor size in more than $50 \%$ of its initial size (Fig. 4).

CONCLUSION

Although PET is not specific for detecting recurrence, it is very helpful in selected cases for scrutinizing tumor edema from recurrence. PET scans are sometimes used in addition to MRI or CT to evaluate brain tumors. After receiving treatment for a brain tumor, PET scans can also be used to detect new tumor growth, scar tissue and necrosis.
P6:127

\section{DIFFUSION-WEIGHTED MR IMAGING (DWI) IN EVALUATION OF BRAIN TUMORS: COMPARISON 1,5T VS. 3.0 T}

\author{
Z. MERHEMIC $^{1}$, F. Gavrankapetanovic ${ }^{2}$, S. Beslic ${ }^{1}$, N. Bilalovic ${ }^{3}$, \\ M.M. Thurnher \\ ${ }^{1}$ Clinical Centre University Sarajevo, Clinic for Radiology, \\ Sarajevo, BOSNIA HERZEGOVINA, ${ }^{2}$ Clinical Centre University \\ Sarajevo, Clinic for Surgery, Sarajevo, BOSNIA HERZEGOVINA, \\ ${ }^{3}$ Clinical Centre University Sarajevo, Clinic for Pathohistology, \\ Sarajevo, BOSNIA HERZEGOVINA, ${ }^{4}$ Medical University of \\ Vienna, Department of Radiology, Vienna, AUSTRIA
}

\section{PURPOSE}

Diffusion-weighted MR imaging is proven useful for vascular disorders, infections and evaluation of brain tumors. The aim of this study was to compare apparent diffusion coefficient (ADC) values of brain tumors measured on $1.5 \mathrm{~T}$ and $3.0 \mathrm{~T}$ MRI.

MATERIALS AND METHODS

Ten patients (four women and six men, range age 17 to 75 years ) with histologically proven brain tumors ( four meningiomas, two glioblastomas, two metastasis, one oligoastrocytoma and one neurocytoma) were examined on 1.5 Tesla and 3.0T unit. In all patients conventional MR sequences and DWI were performed. Three region of interest (ROI) measurements were placed on ADC maps, and the mean ADC value was used for further analysis. RESULTS

The mean ADC of meningiomas was $0.91 \times 10^{-3} \mathrm{~mm} 2 / \mathrm{sec}$ on $1.5 \mathrm{~T}$, and $0.84 \times 10^{-3} \mathrm{~mm} 2 / \mathrm{sec}$ on $3.0 \mathrm{~T}$ unit; of the glioblastomas $1.8 \times$ $10^{-3} \mathrm{~mm} 2 / \mathrm{sec}$ on $1.5 \mathrm{~T}$, and $2.1 \times 10^{-3} \mathrm{~mm} 2 / \mathrm{sec}$ on $3.0 \mathrm{~T}$; of the metastatic lesion $1.2 \times 10^{-3} \mathrm{~mm} 2 / \mathrm{sec} 1.5 \mathrm{~T}$, and $1.1 \times 10^{-3} \mathrm{~mm} 2 / \mathrm{sec}$ on $3.0 \mathrm{~T}$ unit. The mean ADC of oligoastrocytoma was $1.3 \times 10^{-3}$ $\mathrm{mm} 2 / \mathrm{sec}$ on $1.5 \mathrm{~T}$, and $1.5 \times 10^{-3} \mathrm{~mm} 2 / \mathrm{sec}$ on $3.0 \mathrm{~T}$ unit; of the neurocytoma $1.7 \times 10^{-3} \mathrm{~mm} 2 / \mathrm{sec}$ on $1.5 \mathrm{~T}$, and $2.0 \times 10^{-3} \mathrm{~mm} 2 / \mathrm{sec}$ on 3 T MRI unit. Statistical analysis showed good agreement between measurements when comparing 1.5 and 3.0T units.

CONCLUSION

Despite small number of cases in this study, the results of ADC measurements in brain tumors show good agreement between 1.5 T and 3.0 T MRI unit. Further studies with larger patient sample are neceassary for more solid conlusions.

\section{P6:128}

\section{EFFICACY OF GADOBENATE DIMEGLUMINE FOR CONTRAST-ENHANCED MR IMAGING OF THE CNS IN INTRAINDIVIDUAL CROSSOVER STUDIES}

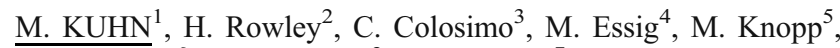
K. Maravilla ${ }^{6}$, H.A. Rowley ${ }^{2}$, Z. Rumbold ${ }^{7}$

${ }^{1}$ University of Illinois at Peoria, Peoria, IL, USA, ${ }^{2}$ University of Wisconsin, Madison, WI, USA, ${ }^{3}$ Catholic University of Sacred Heart, Rome, ITALY, ${ }^{4}$ German Cancer Research Center, Heidelberg, USA, ${ }^{5}$ Ohio State University, Columbus, OH, USA, ${ }^{6}$ University of Washington, Seattle, WA, USA, ${ }^{7}$ Medical University of South Carolina, Charleston, SC, USA 


\section{PURPOSE}

To summarize efficacy results from randomized, double-blind, intraindividual comparisons of gadobenate dimeglumine (GdBOPTA) vs comparators for MRI of CNS lesions.

\section{METHODS}

In 6 studies, 382 patients with CNS lesions received $2 \mathrm{MR}$ exams within 2 days to 2 weeks, one with $0.1 \mathrm{mmol} / \mathrm{kg}$ bodyweight GdBOPTA $(\mathrm{N}=382)$ and one with an equal dose of comparator (gadopentetate dimeglumine or Gd-DTPA [ $\mathrm{N}=237$ ], gadodiamide or Gd-DTPA-BMA [N=117], or gadoterate meglumine or GdDOTA [N=28]). T1WSE and T2W-FSE images were obtained before and T1WSE images after contrast injection. Blinded expert neuroradiologists assessed pre-and postcontrast images for qualitative and quantitative efficacy parameters. The Wilcoxon signed rank test was used to measure study group differences.

\section{RESULTS}

In a pilot study (Colosimo 2001) sensitivity for lesion detection was markedly superior with Gd-BOPTA $(93 \%-100 \%)$ vs comparatorenhanced exams $(65 \%-73 \%)$, with increased lesion-to-brain ration (LBR) observed vs comparators (143\% vs $127 \%$ ). In a follow up study (Colosimo 2004) Gd-BOPTA was preferred over Gd-DOTA $(\mathrm{p}=<0.005)$ for LBR, lesion delineation, internal lesion structure, and overall reader preference. In a subsequent comparison with GdDTPA (Knopp 2004) a significant $(\mathrm{p}<0.05)$ preference for GdBOPTA was noted for global assessment of contrast enhancement, LBR, and all other qualitative parameters. In the largest study to date (157 pts) Maravilla (2006) demonstrated significant $(\mathrm{p}<0.0001)$ preferences for Gd-BOPTA over Gd-DTPA for all diagnostic information endpoints, lesion enhancement, and contrast-to-noise ratio (CNR). In a comparison with Gd-DTPA-BMA (Rowley 2007), a highly significant $(\mathrm{p}<0.0001)$ preference for Gd-BOPTA was demonstrated for all qualitative and quantitative evaluations, including global preference. At 3 T (Rumboldt 2009) Gd-BOPTA was preferred to Gd-DTPA for lesion border delineation and enhancement $(\mathrm{p}<0.001)$, with significantly $(\mathrm{p}<0.05)$ higher LBR, CNR, and $\%$ lesion enhancement.

CONCLUSION

In 6 crossover studies involving 382 patients, Gd-BOPTA consistently resulted in greater contrast enhancement than comparators and was preferred by blinded expert readers.

\section{P6:129}

\section{PERFUSION CT IN EVALUATION OF THE SECONDARY BRAIN TUMOR TREATMENT BY GAMMA KNIFE}

M. DOLGUSHIN $^{1}$, I. Pronin ${ }^{1}$, A. Golanov ${ }^{1}$, L. Fadeeva ${ }^{1}$, A. Turkin ${ }^{1}$, A. Podoprigora $^{1}$, S. Ilyalov ${ }^{1}$, A. Zaytceva ${ }^{2}$, V. Kornienko ${ }^{1}$

${ }^{1}$ N.N.Burdenko Neurosurgical Institute, RAMS, Moscow, RUSSIA,

${ }^{2}$ Clinical Hospital N1, President Medical Centre, Moscow, RUSSIA

Metastasis are high vascularised neoplasms in comparison with the normal brain tissue. Radiosurgery changes tumor hemodynamics due to destruction tumor vascular structures. The aim of this work is using of CT perfusion imaging (PCT) for evaluation of the secondary brain tumor (SBT) before and after radiosurgical (RS) treatment.

\section{MATERIALS \& METHODS}

44 patients (solitar metastasis-12, multifocal-32), underwent MRI and PCT before and after radiosurgery (Leksell Gamma Knife). 14 patients underwent 18 FDG PET. In 5 pts PCT was performed:on the 3-rd, 20-th, 30-th day, 3-rd and 6-th months. PCT protocol: i/v $40 \mathrm{ml}$ of contrast media, $350-370 \mathrm{mg} / \mathrm{ml}$, flow rate- $4 \mathrm{ml} / \mathrm{s}, 4 \times 5 \mathrm{~mm}$ slices. Perfusion maps (Perfusion I) were calculated offline, regions of interest (ROI) were taken in tumor and in contralateral hemisphere. RESULTS

CEMRI demonstrated high contrast enhancement in all cases with SBT. CBV, CBF values were the highest in melanomas $(15 / 03+/-$ $9.7 \mathrm{ml} / 100 \mathrm{~g}, 113.99+/-24.25 \mathrm{ml} / 100 \mathrm{~g} / \mathrm{min})$; MTT values were the longest for metastases kidney and ovarian cancer(18.46+/-1.4 and $10.6+/-6.3 \mathrm{~s})$ before the RS. There were no significant changes in tumor perfusion on the 3-rd day and on 20-th day after RS ( $p>0.05$ ). But mean values of $\mathrm{CBF}, \mathrm{CBV}$ demonstrated more than 3-fold significant $(p<0.04)$ decreasing in SBT ROIs; mean MTT values - 2-fold increasing $(\mathrm{p}<0.5)$ in a month after RS and later. PET data correlated with PCT data. Mean values of PET standardized uptake value (SUV) in SBT varied from 2.8 to 8.6, before RS and from 0.4 to 2.5 after RS. CBV, CBF, MTT values on the 3-rd month decreased to $1.37+/-0.94 \mathrm{ml} / 100 \mathrm{~g}, 20.0+/-$ $12.41 \mathrm{ml} / 100 \mathrm{~g} / \mathrm{min}, 3.6+/-2.6 \mathrm{~s}$ : on the 6-th month to $1.02+/-$ $1.31 \mathrm{ml} / 100 \mathrm{~g}, 14.70+/-9.81 \mathrm{ml} / 100 \mathrm{~g} / \mathrm{min}, 5.4+/-3.8 \mathrm{~s} .9 \mathrm{pts}$ with large multifocal SBT (25-30 $\mathrm{mm}$ in diameter) didn't response to RS and were treated surgically.

\section{CONCLUSION}

PCT is a rapid noninvasive imaging technique, which is informative for evaluation dynamic changes in tumor tissue after RS and it may be an alternative to PET.

\section{P6:130}

\section{PREDICTIVE VALUE OF MRI AND CT FINDINGS IN CHILDHOOD MEDULLOBLASTOMA STUDIED IN THAI PATIENTS}

\section{O. CHAWALPARIT ${ }^{1}$, Y. WATTANASEN ${ }^{1}$, T. SANGRUCHI ${ }^{2}$ \\ ${ }^{1}$ DEPT. RADIOLOGY, FACULTY OF MEDICINE SIRIRAJ HOSPITAL, MAHIDOL UNIVERSITY, BANGKOK, THAILAND, ${ }^{2}$ DEPT. PATHOLOGY, FACULTY OF MEDICINE SIRIRAJ HOSPITAL, MAHIDOL UNIVERSITY, BANGKOK, THAILAND}

\section{OBJECTIVE}

To evaluate the MRI and CT findings as prognostic value of medulloblastoma.

\section{METHODS}

From 1998 to 2007, MRI and CT of fourteen patients with medulloblastoma were retrospectively reviewed. The findings of cyst or necrotic portion, composition of calcification, hemorrhage and residual tumor after surgical removal were evaluated. Histopathologic findings were classified as large cell and non-large cell type. The evidence of leptomeningeal seeding, recurrence event and survival time were used to indicate outcomes of the disease.

\section{RESULT}

Only large cell type of medulloblastoma was statistically significance in decreasing survival time. No other MRI or CT findings were statistically significant to predict the outcomes of disease. 


\section{CONCLUSION}

Histologic type of medulloblastoma is predictive indicator of outcome of treatment regardless imaging findings. However, limitation of this study due to small sample size was considered.

\section{P6:131}

\section{MULTIMODAL MAGNETIC RESONANCE IMAGING INCREASES THE OVERALL DIAGNOSTIC ACCURACY IN BRAIN TUMORS}

K. ABUL-KASIM ${ }^{1}$, M. Thurnher ${ }^{2}$, S. Puchner $^{2}$, A. Overgaard ${ }^{1}$, P. Sundgren ${ }^{1}$

${ }^{1}$ Faculty of Medicine, University of Lund, Malmö, SWEDEN, ${ }^{2}$ Medical University of Vienna, Vienna, AUSTRIA

\section{BACKGROUND AND PURPOSE}

The aim of this study was to assess the contribution of multimodal magnetic resonance imaging (MRI) techniques: diffusion weighted imaging (DWI), perfusion weighted imaging (PWI) and/or magnetic resonance spectroscopy (MRS) to increase the diagnostic accuracy of MRI in brain tumors.

\section{MATERIAL AND METHODS}

Forty one patients with suspected brain tumor [27 patients were male $(66 \%)$, mean age $58.3 \pm 16$ (mean \pm SD) year] were included in this analysis. All patients were examined with conventional MR-sequences, DWI and with PWI and/or MRS. Kappa coefficient was calculated to estimate the concordance of the diagnosis given by multimodal MRI and the diagnosis given the conventional MR-sequences with the final diagnosis obtained by biopsy. Fisher exact test has been performed to estimate the added utility of multimodal MRI. Statistical significance was set to $<0.05$.

RESULTS

With multimodal MRI the diagnosis in 38 patients (93\%) was the same as that obtained by biopsy compared with 14 patients $(34 \%)$ when the readers were allowed to give one diagnostic possibility during the evaluation of the conventional MR-sequences. The difference was statistically significant (Fisher exact test; $\mathrm{P}<0.0001$ ). The concordance between the diagnoses given by evaluating images from multimodal MRI and the final diagnoses was almost perfect (ê value $0.91 ; 95 \%$ confidence interval $0.81-1.01$ ). PWI have primarily helped to differentiate lymphomas from other solid tumors whereas MRS helped to differentiate malignant glioma from metastasis. Both PWI and MRS helped in grading astrocytomas.

\section{CONCLUSION}

Combining the PWI and/or MRS with conventional MR imaging significantly increase the diagnostic accuracy. Therefore multimodal MRI examinations should wherever available be performed in the workup of brain tumors although it means increasing examination cost and time.

\section{P6:132}

\section{RADIATION INDUCED TEMPORAL LOBE NECROSIS: MRI FINDINGS}

F. TAYFUN, A. KEVEN, K. KARAALI, A.U. SENOL AKDENIZ UNIVERCITY MEDICINE FACULTY RADIOLOGY DEPARTMENT, ANTALYA, TURKEY

\section{PURPOSE}

To present MRI findings of radiation induced temporal lobe necrosis in a patient with nasopharynx carcinoma treated by radiotherapy.

\section{METHODS}

A 45-year-old male patient was referred for brain MRI to our institution. His main complaint was headache. The patient was treated for nasopharyngeal carcinoma 3 years ago and had been given radiotherapy.

RESULTS

MR examination revealed inhomogeneously enhancing lesions within both temporal lobes and extensive white matter edema on both cerebral hemispheres. A slight compression of both lateral ventricles due to the edema was also noted. Previous brain MRI exams of the patient were also evaluated. Enhancing lesions appeared 1 year after radiotherapy and showed marked progression within three years. Edema of the white matter also showed progression. These findings were consistent with radiation induced necrosis.

CONCLUSIONS

Radiation induced necrosis is an important complication of radiotherapy. Differentiation of this entity from metastasis and other malignant lesions may sometimes be difficult. History of the patient, the type and the dose of the radiotherapy and chronological evaluation of the MRI exams will help the radiologist make the diagnosis. MR spectroscopy may also be useful. In this presentation, 3 year follow-up findings of the patient are given and key points in the diagnosis are discussed.

\section{P6:133}

\section{MR IMAGING FEATURES OF THE VARIOUS CAVERNOUS SINUS LESIONS AND REVIEW OF THE LITERATURE}

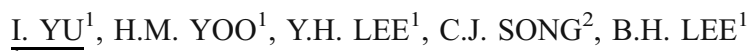 \\ ${ }^{1}$ EULJI UNIVERSITY HOSPITAL, DAEJEON, SOUTH KOREA, ${ }^{2}$ CHUNGNAM UNIVERSITY HOSPITAL, DAEJEON, SOUTH KOREA}

\section{PURPOSE}

This poster presents the MR imaging features of the various cavernous sinus (CS) lesions, including neoplastic, infective, inflammatory, and vascular ones and review the literature.

METHODS

We reviewed the clinical, and MR imaging features of various lesions, involving the CS, including neoplastic lesions(schwannoma, meningioma, hemangioma, epidermoid, invasive pituitary adenoma, lymphoma, chordoma, chondrosarcoma, nasopharyngeal carcinoma, sphenoid carcinoma, metastasis), infective, inflammatory lesions (Tolosa-Hunt syndrome, invasive fungal infection, pachymeningitis), and vascular lesions(CCF, dural AVF, Aneurysm, thrombosis). RESULTS

Schwannomas show usually cystic portions in the tumor. Cavernous hemangiomas usually show homogenous high T2SI, homogenous enhancement. Epidermoid cysts of the CS show high SI on DWI. Malignant tumors, and inflammatory pseudotumors (Tolosa-Hunt syndrome) usually show intermediate to low T2SI, with weak enhancement. Inflammatory, infective, and granulomatous lesions show linear or nodular enhancement of the meninges of the CS, but often have nonspecific MR imaging features. 
Vascular lesions of CS is well detected on MRI or MRA-source images.

\section{CONCLUSION}

MR imaging features of various neoplastic, infective, inflammatory, and vascular lesions, involving the CS are often specific, but some non-specific. In many of these cases, involvement elsewhere suggests the diagnosis. MR imaging is sensitive for detecting vascular lesions of CS. To understand the clinical, MR and features of CS lesions help the accurate diagnosis and the appropriate treatment.

\section{Imaging of the Spine and Spinal Cord}

\section{P7:134}

\section{THE BENEFIT OF DYNA CT IMAGING IN THE CERVICAL OSSIFICATION OF THE POSTERIOR LONGITUDINAL LIGAMENT (OPLL) PATIENTS AT PRASAT NEUROLOGICAL INSTITUTE}

\section{K. SRISUBAT}

PRASAT NEUROLOGICAL INSTITUTE, BANGKOK, THAILAND

Radiographic Imaging from general x-ray and CT Scan are used for diagnosis the ossification of posterior longitudinal ligament (OPLL). Reformatted imaging from CT Scan can well define the outline of OPLL. Moreover, the 3D imaging from CT Scan can demonstrate the shape of OPLL which is used in operation procedure. At present, multi-slice CT can be used widespread rather than a single slice CT. At Prasat Neurological Institute, we have single slice CT that has limitation for scanning and processing. We applied Dyna CT instead. PURPOSE

The purpose of this study is to use a new application rotational angiography system (DYNA CT) to diagnose OPLL.

METHODS

The retrospective of OPLL patients at cervical spine during March 2007 and July 2008 at Prasat Neurological Institute done by Dyna CTwere reviewed. Reformatted in axial, sagittal and coranal with thin slice $2 \mathrm{~mm}$. and 3D volumn rendering by cut and clip tool technique with high resolution can be used.

RESULTS

The imagings from the techniques by Dyna CT can demonstrate the shape and outline of OPLL very clearly.

CONCLUSIONS

Dyna CT can be used to replace single slice CT and it is useful in operation at Prasat Neurological Institute.

\section{$\mathbf{P 7 : 1 3 5}$}

\section{ANOMALIES OF THE CAUDAL CELL MASS: REPORT OF TWO CASES WITH REVIEW OF THE SPINAL CORD EMBRIOLOGY}

\section{SAFRONOVA, I. REGO, J. RESENDE PEREIRA}

Hospital Pedro Hispano - Neurorradiologia, Matosinhos, PORTUGAL

\section{INTRODUCTION}

Caudal cell mass is formed by fusion of the neural epithelium caudal to the posterior neuropore with the notochord. It undergoes secondary neurulation and retrogressive differentiation, becoming the conus medullaris, filum terminale and lower lumbar and sacral nerve roots. A minor anomaly in its formation may result in fibrolipoma of the filum terminale (FFT); a major anomaly may result in regression caudal syndrome (RCS).

\section{CASES REPORT}

Case 1: 1-year-old female referred by a single midline thick hair in the lumbo-sacral region. MRI showed fat in both internum and externum filum segments. The conus medullaris was lying at the level of the L1-L2 disc. The diagnosis of FFT was made.

Case 2: 51-year-old female referred by lower limb neuromuscular atrophy since childhood. The patient had been corrected for exstrophy of the urinary bladder and uterus and presented with a $2 \mathrm{~cm}$ lumbar midline tumefaction covered by skin with hyperthrichosis. CT scan showed spina bifida of all lumbar vertebra and partial sacral agenesis. MRI showed a lipoma under the tumefaction The medulla was tethered on a placod located eccentrically against the lipoma. The placode-lipoma interface was outside the spinal canal, at the L4 level. There was a small meningeal herniation. The diagnosis made was RCS with tethered cord and lipomyelomeningocele.

CONCLUSIONS

FFT and RCS are closed spinal dysraphisms.

FFT results from a minor anomaly of secondary neurulation and retrogressive differentiation. If the filum terminale is too short, a tethered cord syndrome develops. FFT patients should undergo careful neurological examination and urodynamic testing.

RCS results from failed formation of a segment of the notochord with subsequent neural plate failed induction. It is a disorder of gastrulation that also affects the cloaca, causing anomalies of the lower gastrointestinal and genitourinary systems.

Both cases reported demonstrate that the presence of lumbar midline cutaneous stigmata should prompt imaging evaluation of the spinal cord.

\section{P7:136}

\section{BENIGN SOLITARY INVASIVE GIANT PARAVERTEBRAL SCHWANNOMA WITH BONE DESTRUCTION AND BOTH EPIDURAL AND RETROPERITONEAL EXTENSION: CASE REPORT}

N. PETROU SWISS PARAPLEGIC CENTER, DEPARTMENT DIAGNOSTIC RADIOLOGY AND NEURORADIOLOGY, NOTTWIL, SWITZERLAND

\section{PURPOSE}

Paravertebral schwannomas are rare tumors, sometimes with either an intraosseous spread or a retroperitoneal extension. report on an even more rare case of a benign invasive giant paravertebral schwannoma with simultaneously involvement of the vertebral bone and extension to the epidural as well as retroperitoneal space in a 51-year-old man suffered from right side sciatica without stigmata of von recklinghausen's disease.

\section{METHODS}

Presentation of (pre- and postoperative) clinical data and imaging findings (especially magnetic resonance imaging). preoperative selec- 
tive tumor embolisation, operative resection, tumor corpectomy, bone reconstruction and spine stabilisation. histopathologic examination.

\section{RESULTS}

Space occuping, in size $9 \times 7 \times 8 \mathrm{~cm}$ huge lobulated paravertebral invasive soft-tissue mass with spacious expansile bone destruction of vertebra 13 (scalloping; widening of the right neural foramen 13/4) and both epidural (mild spinal canal stenosis) and retroperitoneal (displacement and partial compression of the inferior cava vein) extension. a thoracoabdominal computed tomography showed no evidence of other masses or lymphadenopathy. the initial differential diagnosis included a likely benign lesion (i.e. giant cell tumor; bone cyst; chondroblastoma), though malignancy (i.e. metastasis; plasmocytoma) could not be excluded definitely. the histopathological examination revealed finally a benign schwannoma. at the two-months-followup no neurological deficit in the right lower extremity nor a relevant sciatic pain was found.

\section{CONCLUSIONS}

The rare schwannoma should included in the differential diagnosis of large paravertebral mass with expansile destruction of spine, especially when widening of neural foramen and scalloping of vertebral body is present, even in abscence of von recklinghausen's disease.

\section{KEYWORDS}

Giant schwannoma; bone invasion; lumbar spine; magnetic resonance imaging; computed tomography.

\section{P7:137}

\section{DIFFUSION TENSOR IMAGING IN SPINAL CORD INJURY}

J. PETERSEN $^{1}$, B. Wilm ${ }^{3}$, J. von Meyenburg ${ }^{2}$, M. Schubert ${ }^{1}$, V. Dietz ${ }^{1}$, S. Kollias ${ }^{2}$

${ }^{1}$ University Hospital Balgrist, Spinal Cord Injury Center, Zurich, SWITZERLAND, ${ }^{2}$ University Hospital Zurich, Institute of Neuroradiology, Zurich, SWITZERLAND, ${ }^{3}$ University \& ETH Zurich, Institute for Biomedical Engineerging, Zurich, SWITZERLAND

\section{INTRODUCTION}

Due to poor image quality and limited resolution, Diffusion Tensor Imaging (DTI) has rarely been applied to investigate disorders of the spinal cord. Recent improvements in MR pulse sequence design have overcome these problems, thereby providing a new instrument for the assessment of Spinal cord injury (SCI).

METHODS

DTI data of 19 traumatic SCI subjects (level of lesion C3 to C8, mean age 59.7 years, time since injury 2 months to 8 years) and of 28 healthy volunteers (mean age 58 years) were acquired on a Philips Achieva 3 T MR scanner using an outer volume suppressed reduced field of view (FOV) acquisition with oblique slice excitation and a single-shot EPI readout. Fractional anisotropy (FA) and apparent diffusion coefficient (ADC) maps were calculated at cervical (C2 and C5), thoracic (T5) and lumbar (L1) levels of the spinal cord. Diffusivity values were evaluated in 10 regions of interest (ROIs) comprising the cross sectional area and certain parts of gray and white matter where motor and sensory tracts are located. Surface areas of spinal cord cross sections were compared.

\section{RESULTS AND CONCLUSION}

In SCI subjects, FA values as compared to volunteers were decreased at all four spinal levels. At level $\mathrm{C} 2$, the decrease was statistically significant for the cross sectional area and for motor and sensory tracts $(\mathrm{p}<0.05)$. Surface area of the injured spinal cord was decreased at all levels.

The low FA values in the SCI subjects group are likely to reflect demyelination and axonal degeneration. Significant reduction of FA in the upper cervical segments suggests that regions remote from the injury site might be affected by progressive degeneration distant to the traumatic lesion. The reductions in surface area suggest atrophy. In conclusion, DTI provides useful, quantitative parameters for the objective evaluation of disease progression and potentially for monitoring treatment response.

\section{P7:138}

\section{TUBERCULOUS MYCOTIC PSEUDOANEURYSM OF THE AORTA}

\section{T. PARREIRA, C. CASIMIRO, P. LIMA, G. SANTOS, P. MELO} FREITAS

Hospitais da Universidade de Coimbra - Clínica Universitária de Imagiologia - Neurorradiologia, Coimbra, PORTUGAL

\section{INTRODUCTION}

Tuberculous aneurysm of the aorta is extremely rare. Tubercle bacilli may reach the aortic wall in one of three ways: directly on the internal surface of the vessel wall in regions where the resistance to infection is lowered (eg. by atherosclerosis), the bacilli may be carried to the adventitia or media by the vasa vasorum, or by direct extension from a contiguous focus such as a lymph node or paraspinal abscess.

CASE REPORT

A 75 year-old male was admitted to the hospital ward with a history of fever, weight loss, and disabling back pain. Airway secretions were inconclusive for acid-fast bacilli on smear, and negative on culture for Mycobacterium tuberculosis. Fiberoptic bronchoscopy was also negative. Chest CT showed two cavitated lesions and sparse opacifications consistent with an inflammatory process. Back pain was studied with dorsal CT and MRI, revealing an expansile / destructive lesion centered on D9-D10 with intracanalar and paravertebral extension, associated with a pseudoaneurysm of the dorsal aorta. A favorable clinical outcome was reached with tuberculostatic therapy. He awaits surgical decision for the vascular lesion. CONCLUSION

The diagnosis was only possible through complete clinical / radiologic integration, and was confirmed by the favorable patient outcome on tuberculostatics. The aorta involvement is rare. In the described case, it probably occurred through direct vessel wall erosion by the infectious dorsal lesion.

\section{P7:139}

\section{CLINICALLY SILENT BRAIN AND SPINE TUBERCULOSIS}

\section{B. MOREIRA, P. PINTO, C. VASCONCELOS, R. CRUZ}

Hospital Geral de Santo Antonio - Neuroradiology Department, PORTO, PORTUGAL

\section{INTRODUCTION}

Diagnosis of extrapulmonary tuberculosis is often difficult, as it can mimic a number of other disease entities. We present a case of 
disseminated and clinically silent brain and spine tuberculosis, in which the spine radiographic findings were the key to the diagnosis.

CASE REPORT

A 26-year-old man was taken to the ER department after a seizure. A history of seizures was reported since he was 6-months-old and, in the previous year, he had also been diagnosed with intravenous drug abuse related $\mathrm{HCV}$ infection.

$\mathrm{He}$ recovered from the pos-ictal state within one hour and the neurological exam was unremarkable. Nevertheless, as there were external signs of trauma, the ER physician suggested performing a brain CT that showed linear calcifications along the left perirolandic cortex. It was also noticed a subcortical right parietal lesion with mild enhancement and edema, as well as a right occipital lytic lesion.

In the MR scan, there was linear cortical enhancement in the left perirolandic lesion that was presumed to correspond to meningioangiomatosis. The parietal lesion showed peripheral enhancement and diffusion restriction, suggesting an abscess.

As he complained about abdominal pain, an abdominal CT was also performed. It disclosed multiple osteolytic lesions in the thoracic and lumbar spine with sclerotic margins and central calcification.

A granulomatous disease was suspected and a culture from a prevertebral abscess was performed, confirming the diagnosis of mycobacterium tuberculosis infection.

COMMENTS

In this case, the particular lesions the patient had through the spine and skull base were the clues to the diagnosis. It is important to be familiar with the various radiologic features of tuberculosis to ensure early accurate diagnosis.

P7:140

\section{MR DIFFUSION TENSOR IMAGING OF THE CERVICAL SPINAL CORD: CORRELATION WITH CLINICAL AND ELECTROPHYSIOLOGY FINDINGS IN PATIENTS WITH SPONDYLOTIC SPINAL CORD COMPRESSION}

\author{
M. KERKOVSKY $^{1}$, J. BEDNARIK ${ }^{2}$, A. SPRLAKOVA - PUKOVA ${ }^{1}$, \\ I. URBANEK $^{2}, \mathrm{M}$. $\mathrm{MECHL}^{1}$ \\ ${ }^{1}$ University Hospital Brno and Masaryk University - Department \\ of Radiology, Brno, CZECH REPUBLIC, ${ }^{2}$ University Hospital \\ Brno and Masaryk University - Department of Neurology, Brno, \\ CZECH REPUBLIC
}

\section{PURPOSE}

Spondylotic cervical myelopathy (SCM) represents an important clinical issue. The imaging diagnosis is currently based on conventional MR examination; however, interpretation of the MR findings in relation to a clinical picture may be difficult. In our paper we present preliminary data of a study aimed at evaluation of diffusion tensor imaging (DTI) in spondylotic cervical spinal cord compression in relation to clinical and electrophysiology findings.

METHODS

A group of 37 patients with MR findings of cervical spinal cord compression were examined on $1,5 \mathrm{~T}$ scanner, the protocol involved axial DTI slices. The whole group was divided into subgroups according to clinical signs of myelopathy and electrophysiology findings. Fractional anisotropy (FA) and apparent diffusion coefficient (ADC) of the spinal cord cross sections were measured. We compared the $\mathrm{C} 2 / 3$ level without compression (NCL) and the level of maximal spinal cord compression (MCL) using parametric testing (t-test). We also compared ADC and FA values at MCL between the subgroups of patients distinguished either by the clinical manifestation of myelopathy or the electrophysiology findings.

RESULTS

The average FA value was lower at MCL $(0.5)$ compared to NCL $(0.585)$ within the group of all patients $\left(\mathrm{p}=1 \times 10^{-7}\right)$, also ADC values decreased significantly at MCL. The spinal cord FA values at MCL were lower in symptomatic myelopathy subgroup compared to asymptomatic subjects ( 0.457 and 0.527 , respectively; $p=0.007)$, while the ADC values were higher in the symptomatic patients (1.324) compared to asymptomatic $(1.135)$ patients $(p=0.01)$. The observed parameters did not change significantly with respect to electrophysiological abnormalities.

CONCLUSIONS

The preliminary data document the sensitivity of the DTI sequence to spinal cord compression with significant decrease of FA and ADC values at the compressed level. The observed parameters did not correlate with electrophysiology findings, but the results suggest the DTI to reflect the clinical manifestation of SCM.

\section{P7:141}

\section{DIFFUSION TENSOR IMAGING OF THE CERVICAL SPINE IN PATIENTS WITH MULTIPLE SCLEROSIS}

A. CABRERA ZUBIZARRETA ${ }^{1}$, I. SARALEGUI PRIETO ${ }^{1}$, M. MENDIBE BILBAO ${ }^{2}$, M.B. FERNANDEZ RUANOVA ${ }^{1}$, J.M. ONTAÑON GARCES ${ }^{1}$, I. VICENTE OLABARRIA ${ }^{1}$

${ }^{1}$ GALDAKAO HOSPITAL. OSATEK.SA. MR UNIT, GALDAKAO-BILBAO, SPAIN, ${ }^{2}$ CRUCES HOSPITAL. NEUROLOGY DEPARTMENT, BARAKALDO-BILBAO, SPAIN

\section{PURPOSE}

To evaluate the DTI features in different regions and levels of the cervical spinal cord in patients with mutiple sclerosis.

METHOD

We studied 18 patients (13 M/5F; m36.5Y) with multiple sclerosis (16RRMS, 1PPMS and 1SPMS) and 11 sex and age matched controls. MR was performed at $1.5 \mathrm{~T}$. MRI examination included axial gradient T2, sagital Turbo Spin Echo T2, Fast Stir and pulsed gated PD weighted images. DTI sequence was adquired in the axial plane using pulsed gradient echo planar imaging (TR/TE 4200/87; IPAT 2;matrix $128 \times 128$; FOV $200 \times 200 ; 304 \mathrm{~mm}$ slices; $\mathrm{b}=900$ ) Diffusion weighting was applied along 20 non collinear directions and quantitative analysis was performed using vendor software. ADC and FA were calculated on a ROI basis on the lateral and posterior columns and central spinal cord at three levels: C2, C4-C5 and C7-T1.

RESULTS

FA values in controls ranged from 0.946 to 0.600 at $\mathrm{C} 2$ level; from 0.918 to 0.603 at C4-C5 and from 0.790 to 0.512 at C7-T1 
ADC values in controls ranged from 0.560 to $0.916 \times 10^{-3} \mathrm{~mm}^{2} / \mathrm{sg}$ at $\mathrm{C} 2$ level; from 0.718 to $1.287 \times 10^{-3} \mathrm{~mm}^{2} / \mathrm{sg}$ at $\mathrm{C} 4-\mathrm{C} 5$ and from 0.845 to $1.344 \times 10^{-3} \mathrm{~mm}^{2} / \mathrm{sg}$ at $\mathrm{C} 7-\mathrm{T} 1$.

FA values in patients ranged from 0.943 to 0.513 at $\mathrm{C} 2$ level; from 0.856 to 0.455 at $\mathrm{C} 4-\mathrm{C} 5$ and from 0.785 to 0.273 at $\mathrm{C} 7-\mathrm{T} 1$

$\mathrm{ADC}$ values in patients ranged from 0.632 to $1.247 \times 10^{-3} \mathrm{~mm}^{2} / \mathrm{sg}$ at $\mathrm{C} 2$ level; from 0.702 to $1.412 \times 10^{-3} \mathrm{~mm}^{2} / \mathrm{sg}$ at $\mathrm{C} 4-\mathrm{C} 5$ and from 0.876 to $1.795 \times 10^{-3} \mathrm{~mm}^{2} / \mathrm{sg}$ at C7-T1.

Significant differences were found between patients and controls in the right ( $\mathrm{ADC} \mathrm{P} 0.04$ ) lateral column at $\mathrm{C} 2$; right ( $\mathrm{ADC}$ $\mathrm{P} 0.013)$ and left (ADC P0.05) posterior columns and rigth (ADC P0.02) and left (FA P0.014 ADC P0.04) lateral column at C7-T1. CONCLUSION

DTI shows additional data which may be useful in the clinical management of MS patients

\section{P7:142}

\section{PERFUSION CT OF THE SPINAL CORD: FEASIBILITY, REPRODUCIBILITY OF THE METHOD, AND INTERCHANGEABILITY OF THE PERFUSION VALUES BETWEEN TWO SOFTWARE PACKAGES}

$\underline{\text { K. Surlan-Popovic }}^{1}$, Z. Rumboldt ${ }^{2}$, M.V. Spampinato ${ }^{2}$, T. Plotas ${ }^{3}$, S. Bisdas ${ }^{4}$

${ }^{1}$ Clinical Centre Ljubljana, Ljubljana, SLOVENIA, ${ }^{2}$ Medical University of South Carolina, Charleston, USA, ${ }^{3} \mathrm{CT}$ and MRI Peloponnisou, Corinth, GREECE, ${ }^{4}$ Eberhard Karls University Hospital, Tübingen, GERMANY

\section{PURPOSE}

To examine the feasibility of perfusion CT (PCT) of the cervical spinal cord, to examine the reproducibility of the technique, and the interchangeability of the values obtained by software packages.

METHODS

The PCTs of 50 patients with tumors in the head and neck were post-processed using two software packages (Software 1: deconvolution-based analysis with adiabatic tissue homogeneity approach and Software 2: maximum-slope-model with Patlak analysis). Twelve patients were examined twice after a median of $46 \mathrm{~h}$ in order to assess the method reproducibility. Two neuroradiologists separately generated two sets of calculation for each patient, corresponding to two arterial input functions (AIFs): (i) the larger of the two internal carotid arteries (ICA) and (ii) the vertebral artery (VA). Maps that represented blood flow (F), blood volume (V), mean transit time (MTT), time to peak (tp), and permeability (PS) were generated. Comparison tests, measurement reproducibility and inter-observer agreement tests were conducted. RESULTS

The medians of F, V, MTT, PS, and tp parameters obtained with the Software 1 with AIF in the ICA as well as AIF in the VA were $6.1,1.0,14.9,1.1,4.4$ and $6.8,1.2,15.6,0.8,5.2$ respectively. $\mathrm{V}$ and MTT values revealed significant differences between the two AIFs ( $p$-value $=0.03$ ). With the Software 2 the $F$ and $V$ values were different between the two AIFs ( $p$-value=0.02). The interobserver agreement was excellent. The reproducibility was satisfactory. The significant changes for a single patient (\%) using Software 1 were $\pm 120 \%, 90 \%, 180 \%$, and $250 \%$ for F, V, MTT, and PS; using Software 2 were $\pm 110 \%, 80 \%$, and $130 \%$ for F, V, and PS, respectively. The interchangeability between the two packages was acceptable for F and PS.

CONCLUSIONS

PCT of the cervical spinal cord is feasible and reproducible. The values are dependent on the AIF and there is limited interchangeability.

\section{P7:143}

\section{BROWN TUMOR OF SPINE CAUSING CORD COMPRESSION-CASE REPORT AND REVIEW OF THE LITERATURE}

\section{J. LUCKMAN ${ }^{1}$, S. Maimon ${ }^{2}$, D. Ben Ami ${ }^{3}$}

${ }^{1}$ Rabin Medical center, Petah Tikva, ISRAEL, ${ }^{2}$ Soraski medical center, Tel aviv, ISRAEL, ${ }^{3}$ Wolfson medical center, Holon, ISRAEL

\section{PURPOSE}

To describe this rare pathology and imaging and review the literature. METHODS

y.s. is a 50 year old patient on chronic hemodialisis due to CRF of unknown reason, who presented to neurology due to bilateral lower extremity weakness and partial loss of sphincteric power for a few days.

On cervical and thoracic MRI that was obtained 24 hours after admission a few Expansile but non destructive lesions were visualised at T2-3 and T5 levels within the spinous process and lamina.

The lesion at T2 showed evidence of significant canal narrowing, The patient was operated on tyhe same day with laminectomy and decompression and did well clinicaly.

RESULTS AND CONCLUSIONS

Brown tumors of hyperparathyroidism are uncommon lesions in the spine to cause cord compression.

Tertiary hyperparathyroidism is not uncommon in dialisis patients and brown tumors should be raised as a differntial diagnosis for lytic expansile lesions in the skeleton.

There are a few case reports in the litrature but there is no thourough description of the imaging findings on MR.

The findings are not specific but should be suggestive of the diagnosis in the right clinical setting.

The imaging findings will be visualised and thourougly described and discussed.

\section{P7:144}

\section{IMAGING FINDINGS OF SPINAL INTRADURAL TUMORS: A 12-YEARS LONG SERIES}

$\underline{\text { P. Bastos Lima }}{ }^{1}$, F. Cabrita ${ }^{2}$, T. Parreira ${ }^{1}$, C. Marques ${ }^{1}$, C. Casimiro $^{1}$, J. Gonçalves ${ }^{2}$, O. Rebelo ${ }^{3}$, F. Rio $^{1}$

${ }^{1}$ Neuroradiology Unit - Department of Imagiology - Hospitais da Universidade de Coimbra, Coimbra, Portugal, ${ }^{2}$ Neurosurgery Hospitais da Universidade de Coimbra, Coimbra, Portugal, ${ }^{3}$ Neuropathology - Hospitais da Universidade de Coimbra, Coimbra, Portugal

\section{INTRODUCTION}

Intradural tumours are rare even in referral centres. Prompt and appropriate imaging plays a central role in obtaining timely and accurate diagnosis. MRI is particularly useful in these lesions, 
which have high morbidity and often require expert surgical treatment. Pre-operative differential diagnosis, relies heavily on imaging data, such as location (intradural versus extradural) and signal characteristics (before and after intravenous contrast media); which must be interpreted attending to the patient's age, gender and clinical presentation.

\section{OBJECTIVE}

To perform a review of all intradural tumours observed between 1997 and 2008. To collect and contrast imaging features with histopathological results of all cases.

METHOD

We conducted a retrospective analysis, collecting data form clinical, radiological and histopathological records of patients with intradural tumours treated in a twelve-year period, from 1997 to 2008.

\section{RESULTS}

62 case files were recovered, of which $44(70,9 \%)$ belonged to female patients. The average age at presentation was 54,3 years $(\mathrm{SD} \pm$ 14,4 years). We report imaging findings in 12 schwannomas, 14 meningiomas, 1 neurofibroma, 6 ependymomas, 2 myxopapillary ependymomas, 6 astrocytomas, 2 hemangioblastomas, 1 ganglioglioma, 1 leiomyoma, and 1 metastasis of a systemic tumour. CONCLUSION

With the exception of a single extremely rare tumour, the overall composition our series is comparable to what has been reported in similar reviews. The significant volume of data advanced on the appearance of these lesions illustrates the diverse features which may be present on imaging and their usefulness for diagnostic purposes. As expected, MRI was found to be of paramount importance in all cases, providing the largest contribution. Surprising or otherwise unexpected diagnosis are presented and discussed individually.

\section{P7:145}

\section{Brachial neuritis caused by Varicella-Zoster diagnosed by changes in brachial plexus on MRI}

\author{
T. Ayoub ${ }^{1}$, Z. Tahir ${ }^{2}$, V. Raman ${ }^{3}$, M. Chowdhury ${ }^{4}$ \\ ${ }^{1}$ Guys \& St. Thomas Hospital, London SE1 7EH, United \\ Kingdom, ${ }^{2}$ Kingston Hospital, Kingston-Upon-Thames, Surrey, \\ United Kingdom, ${ }^{3}$ Consultant, Department of Radiology, \\ Conquest Hospital, The Ridge, St Leonards-on-sea, East Sussex, \\ United Kingdom, ${ }^{4}$ Consultant, Department of Neurology, \\ Conquest Hospital, The Ridge, St Leonards-on-sea, East Sussex, \\ United Kingdom
}

Brachial neuritis is a rare disorder affecting the brachial plexus. It is characterized by the acute onset of shoulder and arm pain followed by weakness, sensory loss and atrophy. Diagnosis is essentially clinical with electrophysiological investigations and imaging useful in excluding other differentials and supporting the diagnosis. Magnetic resonance imaging (MRI) usually does not show any pathology in the brachial plexus or spinal cord. We present a case of a patient who had brachial neuritis preceded by varicella zoster infection. This was supported by MRI which showed abnormal signal consistent with inflammatory changes in the brachial plexus - to our knowledge a first in the literature.

\section{P7:146}

\section{MRI AND PATHOLOGY FINDINGS IN ACUTE SPINAL CORD COMPRESSION DUE TO EPIDURAL LIPOMATOSIS COMPLICATED BY AN ABSCESS}

\author{
G. ZUCCOLI $^{1}$, N. PIPITONE ${ }^{2}$, N. DE CARLI ${ }^{3}$, L. VECCHIA ${ }^{2}$, \\ S. BARTOLETTI ${ }^{1}$ \\ ${ }^{1}$ University of Pittsburgh, PA, USA, ${ }^{2}$ Arcispedale Santa Maria \\ Nuova, Reggio E., ITALY, ${ }^{3}$ Hospital S. Giuseppe, Empoli, ITALY
}

\section{PURPOSE}

Spinal epidural abscess (SEA) is a rare condition associated with high morbidity and mortality if untreated. SEA is characterized by a collection of pus within the epidural space, between the dura mater and the adjacent fatty tissue. The reported incidence of SEA has been increasing over the last few decades probably as a result of the worldwide diffusion of better spinal imaging procedures. The purpose of our study was to describe the MRI and pathology findings in a case of acute spinal cord compression due to epidural lipomatosis complicated by an abscess. METHODS

Multiplanar and multiecho MR imaging of the total spine was performed emphasizing the relative $\mathrm{T} 1$ and $\mathrm{T} 2$ and $\mathrm{T} 1$ fat saturation signal characteristics. Clinical data of the patient and pathology findings were obtained.

RESULTS

A 68 year-old male without recognized risk factors for infection or spinal epidural lipomatosis presented with rapidly progressive paraplegia of the lower limbs. MR images of the thoracic spine were interpreted as being consistent with an abscess within an epidural lipomatosis compressing the spinal cord. Laminectomy was performed, and a large amount of pus was drained from the epidural lipomatosis, from which Staphylococcus Aureus was isolated. This is the first reported case of an abscess involving an epidural lipomatosis. CONCLUSION

This case is peculiar because: 1) our patient had no known risk factors for spinal infection, 2) there were no classical predisposing factors for spinal lipomatosis and 3) lipoma is usually not prone to become infected. This is the first reported case of an abscess involving an epidural lipomatosis.

\section{Inflammatory, Demyelinating and Degenerative Diseases of the Brain}

\section{P8:147}

\section{APPARENT DIFFUSION COEFFICIENT VALUES CHANGES IN BRAIN ABSCESSES DURING TREATMENT}

T. GERUKIS ${ }^{1}$, D. VOULTSINOU ${ }^{1}$, K. ANASTASIADOU ${ }^{1}$, M. PILAVAKI ${ }^{1}$, V. KALPAKIDIS ${ }^{1}$, P. PALLADAS ${ }^{1}$, P. PRASOPOULOS ${ }^{2}$

${ }^{1}$ Radiology department-GPH G. Papanikolaou Hospital of Thessaloniki, THESSALONIKI, GREECE, ${ }^{2}$ Radiology department, University Hospital of Alexandroupolis, ALEXANDROUPOLIS, GREECE

\section{PURPOSE}

To describe the post-treatment changes in brain abscesses signal intensity on diffusion - weighted imaging (DWI), by measuring the apparent diffusion coefficient (ADC) during successful treatment. 


\section{METHODS}

Three patients with brain abscesses, complicating Non Hodgkin Lymphoma (NHL) in two patients or myelodysplastic syndrome in one patient, treated conservative with antibiotic therapy were evaluated by MRI. The patient was examined at the time of diagnosis (first MRI examination), after two(second MRI examination), four week post- treatment (third MRI examination), and at the remission of the disease (fourth MRI examination. Pre- and post-contrast enhanced T1-W, T2-W, FLAIR were acquired as well as single-shot echo-planar DWI with apparent diffusion coefficient (ADC) mapping.

\section{RESULTS}

At the first MRI examination the mean ADC values were $0.4+-$ $0.7\left(10^{-3} \mathrm{~mm} 2 / \mathrm{sec}\right)$, decreased compared to contralateral normal white matter brain tissue mean ADC values $0.69+-0.21$. ADC values increased between the first MRI examination $0.4+-0.7(10$ $3 \mathrm{~mm} 2 / \mathrm{sec}$ ) and the second MRI examination (2 weeks after antibiotic treatment) $0.7+-0.4(10-3 \mathrm{~mm} 2 / \mathrm{sec})$. ADC values were also increased between the second MRI examination $0.7+-0.4$ $\left(10^{-3} \mathrm{~mm} 2 / \mathrm{sec}\right)$ and the third MRI examination (4 weeks after antibiotic treatment) $1+-1.2\left(10^{-3} \mathrm{~mm} 2 / \mathrm{sec}\right)$, followed by an improvement of the clinical status of the patient.

CONCLUSIONS

ADC values are progressively increased during successful antibiotic treatment and may be helpful in monitoring response to treatment.

\section{P8:148}

\section{TWO CASES OF SEMANTIC DEMENTIA}

N. Kitamura, M. Oka, S. Nakano, S. Kasuya, H. Kudo, R. Kasai, T. Nakatsuka, H. Morita, T. Hasebe, H. Terada

Toho University Sakura Medical Center, Department of Radiology, Sakura, JAPAN

Semantic dementia is a unique frontotemporal lobar degeneration characterized by the loss of meaning or knowledge for words and objects. We used magnetic resonance imaging (MRI) and voxelbased morphometry to assess atrophy, and single photon emission tomography (SPCET) and statistical parametric mapping to examine functional changes in two cases of semantic dementia (SD). The main alterations concerned the left temporal lobe, in accordance with the striking impairment of semantic memory in SD patients. Hypoperfusion was more extensive than grey matter loss in both temporal lobes. While SPECT is more sensitive than MRI, there is striking concordance between morphological and functional abnormalities, which contrasts with the discordance observed in Alzheimer's disease and might be a typical feature of SD.

\section{P8:149}

\section{BRAIN MR VOLUMETRY IN MULTPLEX SCLEROSIS: USE OF OF 3D-FLAIR AND MPRAGE SEQUENCES}

\author{
T. STOSIC-OPINCAL ${ }^{1}$, S. LAVRNIC ${ }^{1}$, M. GAVRLOV ${ }^{1}$, \\ K. DAVIDOVIC ${ }^{1}$, M. DAKOVIC ${ }^{2}$ \\ ${ }^{1}$ Clincal Center of Serbia, Center for Magnetic Resonance, \\ Belgrade, SERBIA, ${ }^{2}$ University of Belgrade, Faculty of Physical \\ Chemistry, Belgrade, SERBIA
}

Standard protocols for determination of brain volume affected by multiplex sclerosis (MS) involve use of images obtained with MPRAGE sequence. However, lesions close to CSF spaces can be overlooked by use of this technique, which can lead to underestimation of volume of MS plaques. The aim of this work is to improve of accuracy of brain extraction and segmentation techniques, which are essential steps in volumetric measurements, using images obtained by both 3D-FLAIR and MPR sequence.

Software packages for structrural brain morphometry (SIENNA) and voxel based morphometry (SPM5) were used in order to evaluate changes in major brain structures caused by MS. As input images of sagital sections obtained with MPRAGE (TR/TE $=2000 /$ $5 \mathrm{~ms}$ ) and 3D-FLAIR (TR/TE $=6000 / 2200 / 300 \mathrm{~ms}$ ) were used; slice thickness were $0.9 \mathrm{~mm}$ and $1.5 \mathrm{~mm}$ respectively. All scans were performed on SIEMENS AVANTO TIM 1.5 MRI device. Obtained results were discussed in terms of reliability of brain extraction from surrounding structures and segmentation of brain tissues.

The use of images obtained using 3D-FLAIR and MPRAGE sequences, showed improved accuracy of results of brain extraction and white mater/gray matter/CSF segmentation. Combination of those sequences as bimodal input in SIENNA and SMP5, in comparison with use of standalone sequences, gave better and more reliable results.

\section{P8:150}

\section{IDIOPATHIC INFLAMMATORY DEMYELINATING DISEASES OF THE BRAINSTEM}

\author{
A. ROVIRA $^{1}$, A. Rovira Gols ${ }^{2}$, J. Sastre-Garriga ${ }^{1}$, C. Auger ${ }^{1}$, \\ J. Munuera' $^{1}$ X. Montalban ${ }^{1}$ \\ ${ }^{1}$ Hospital Vall d'Hebron, Barcelona, SPAIN, ${ }^{2}$ UDIAT. Parc Tauli, \\ Sabadell, SPAIN
}

\section{PURPOSE}

To review the MR imaging features of different idiopathic inflammatory demyelinating diseases that may affect the brainstem.

\section{METHODS}

In this exhibit we will review the clinical and imaging features of the different forms of idiopathic inflammatory-demyelinating diseases (IIDDs) which may involve the brainstem, such as clinically isolated syndromes of the brainstem, multiple sclerosis, neuromyelitis optica and acute disseminated encephalitis.

\section{RESULTS}

Brainstem inflammatory-demyelinating syndrome is frequently the first clinical manifestation of multiple sclerosis, although this condition can also continue as a monophasic disease. In clinically definite multiple sclerosis the brainstem is frequently involved leading to acute clinical syndromes, such as trigeminal neuralgia and internuclear ophthalmoplegia. Later on, chronic damage to the brainstem causes chronic disabling symptoms such as oculomotor disturbances. Most brainstem lesions are contiguous with the cisternal or ventricular cerebrospinal fluid spaces, and range from large confluent patches to solitary, well-delineated paramedian lesions or discrete linings of the cerebrospinal fluid border zones. Predilection for these areas is a key feature that helps to identify 
MS plaques and to differentiate them from focal areas of ischemic demyelination and infarction that preferentially involve the central pontine white matter. Bickerstaff encephalitis is a rare form of acute brainstem syndrome considered a subgroup of acute disseminated encephalomyelitis, in which inflammation appears to be confined to the brainstem. Devic neuromyelitis optica is an uncommon and topographically restricted form of IIDD that is best considered to be a distinct disease rather than a variant of MS. $\mathrm{NMO}$ is characterized by severe unilateral or bilateral optic neuritis and complete transverse myelitis, which sometimes show brainstem involvement.

CONCLUSION

The brainstem may be involved in different forms of idiopathic inflammatory demyelinating diseases. The MRI lesional pattern of these lesions may help in suggesting the diagnosis.

\section{P8:151}

\section{MR IMAGING OF THE BRAIN IN SUSPECTED CONGENITAL MUSCULAR DYSTROPHY: THE COST EFFECTIVE WAY}

\author{
I. Rego ${ }^{1}$, M. Safronova ${ }^{1}$, A. Castro ${ }^{2}$, J.R. Pereira ${ }^{1}$ \\ ${ }^{1}$ Hospital Pedro Hispano. Neurorradiology Department, Matosinhos, \\ PORTUGAL, ${ }^{2}$ Hospital Pedro Hispano. Pediatrics Department, \\ Matosinhos, PORTUGAL
}

\section{INTRODUCTION}

Congenital muscular dystrophies (CMD) are autossomal recessive diseases presenting in the first few months of life. They represent a heterogeneous group of disorders, with different aetiologies, being characterized by hypotonia at birth, muscle weakness and dystrophic changes on muscle biopsy. These diseases may be associated with brain and/or eye anomalies. The CMD with merosin deficiency (CMD1A) is characterized by severe hipotonia and markedly raised creatin kinase on blood serum. It was previously thought to course without central nervous system abnormalities. The advent of CT and MRI showed that characteristic white matter abnormalities are present.

The definite diagnosis is nowadays done by the conjunction of clinical signs and symptoms with brain MR images and muscle biopsy results.

CASE REPORT

A newborn referred by the neonatal unit with axial hipotonia, hiporreactivity and feeding difficulties, with no other anomalies on physical or neurological examination. During the first days of life a progressive increase in $\mathrm{CK}$ values was noted.

The MR revealed a diffuse, symmetrical, supratentorial white matter hiperintensity on T2-weighted images with U-fibbers involvement. A discrete hipersignal on the cerebellar dentate nuclei was also present. A diagnosis of CMD was suggested, namely with merosin deficiency. Skeletal muscle biopsy confirmed the diagnosis of merosin deficient CMD.

\section{CONCLUSION}

Clinical signs and symptoms of CMD should prompt MR imaging of the brain. The finding of characteristic patterns allows cost effective laboratorial workup.

\section{P8:152}

\section{BRAIN MRI IN THE EARLY STAGES OF PARKINSON'S DISEASE AND ATYPICAL PARKINSONIAN SYNDROMES}

\author{
F.J.A. Meijer ${ }^{1}$, W.F. $\mathrm{Abdo}^{2}$, B.R. Bloem ${ }^{2}$, M.M. Verbeek $^{2}$, \\ G.F. Borm ${ }^{3}$, B. Goraj ${ }^{1}$ \\ ${ }^{1}$ University Medical Center St. Radboud, Department of radiology, \\ Nijmegen, THE NETHERLANDS, ${ }^{2}$ University Medical Center St. \\ Radboud, Department of neurology, Nijmegen, THE NETHER- \\ LANDS, ${ }^{3}$ University Medical Center St. Radboud, Department of \\ epidemiology, Nijmegen, THE NETHERLANDS
}

\section{PURPOSE}

Clinical diagnostic criteria of atypical parkinsonian syndromes are often suboptimal or partially validated. The added diagnostic value of brain MRI signs and abnormalities remain particularly unknown in early stages of disease. The goal of our study is to establish the value of brain MRI in the early differential diagnosis of Parkinson's disease and atypical parkinsonian syndromes. METHODS AND MATERIALS

Fifty patients were prospectively included. Accepted international clinical diagnostic criteria were applied. All patients received an MRI at first presentation which were evaluated by two readers. Putaminal signal changes, putaminal atrophy, frontal lobe and parietal lobe atrophy, ventricle dilatation, hummingbird sign, hot cross bun sign, white matter changes, dilated perivascular spaces and lacunair infarctions were scored. Interobserver agreement was evaluated by the kappa coefficient. MRI results were correlated with clinical data and biochemical criteria. The area under the receiver operating characteristic curve (AUC) was used to evaluate the performance of the MRI abnormalities to discriminate between Parkinson's disease and atypical parkinsonian syndromes.

RESULTS

A range of interobserver agreement was seen for the MRI abnormalities. The AUC of the MRI abnormalities will be presented. Midbrain atrophy, pontine signal changes and cerebellar abnormalities show correlations to discriminate between Parkinson's disease and atypical parkinsonian syndromes.

CONCLUSION

Brain MRI in the early stages of disease can be helpful to discriminate between Parkinson's disease and atypical parkinsonian syndromes.

\section{P8:153}

\section{IMMUNE RECONSTITUTION INFLAMMATORY SYNDROME (IRIS) IN AIDS-RELATED PROGRESSIVE MULTIFOCAL LEUKOENCEPHALOPATHY (PML): REPORT OF TWO CASES}

\author{
S. LYRA $^{1}$, S. DAI ${ }^{1}$, A. PAPATHEODOROU ${ }^{1}$, M. CHINI $^{2}$, \\ $\overline{\text { A. LIONI }}^{2}$, M.K. LAZANAS ${ }^{2}$, N. BATAKIS ${ }^{1}$ \\ ${ }^{1}$ Hellenic Red Cross Hospital, Radiology Department, Athens, \\ GREECE, ${ }^{2}$ Hellenic Red Cross Hospital, Infectious Diseases Unit, \\ Athens, GREECE
}




\section{PURPOSE}

Report the occurrence of IRIS in two HIV-infected patients under highly active antiretroviral therapy (HAART).

METHODS

Both patients were male (age 44 years and 35 years respectively). The first patient (stage C3) was receiving HAART for two and a half months. He was admitted in our Hospital with status epilepticus and behavioral disturbances.

The second patient (stage $\mathrm{C} 3$ ) was receiving HAART for three and a half months and was admitted in our Hospital with seizures, behavioral disturbances and cognitive impairment. Both had brain MRI scans.

\section{RESULTS}

MR work-up of the first patient demonstrated PML lesions and the presence of additional small enhancing foci. Follow-up MR examinations demonstrated worsening findings. The patient received corticosteroids and his clinical condition was improved. A 6 month follow-up MRI showed stability of PML lesions (non enhancing foci).

The second patient's initial MRI findings revealed deterioration of preexisting PML lesions. The patient received corticosteroids but his clinical condition remained unchanged. A 4-month follow-up MRI determined no change of the lesions.

\section{CONCLUSIONS}

IRIS associated with PML must be included in the differential diagnosis of HIV-patients presenting with deterioration of mental status and focal neurologic deficits during antiretroviral treatment.

\section{P8:154}

\section{CASE REPORT OF WEGENER'S GRANULOMATOSIS PRESENTING WITH CRANIAL SYMPTOMS}

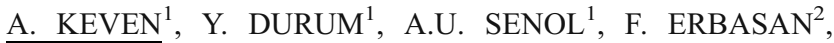
E. TERZIOGLU ${ }^{2}$

${ }^{1}$ Akdeniz University Radiology Department, Antalya, TURKEY,

${ }^{2}$ Akdeniz University Rheumatoloy Department, Antalya, TURKEY

\section{PURPOSE}

We aimed to present a case of wegener's granülomatosis with meningeal involvement.

\section{MATERIAL-METHODS}

MRI of a patient 47 year-old with wegener granülomatosis presenting with neurologic symptoms was performed. Patient had headache and dysarthria.

\section{RESULTS}

T2 Flair weighted MRI study was revaled, there were leptomeningeal and dural enhancement and in periventricüler and subcortical white matter there were nonenhancing white matter lesions.

\section{CONCLUSIONS}

MR imaging demonstrated the wide spectrum of findings of central nervous system involvement in patients with wegener granulomatosis.

\section{P8:155}

\section{IMAGING FINDINGS OF NEUROLISTERIOSIS}

\author{
$\frac{\text { P. } \text { BRESTAS }^{1}}{\text { C. V.K. KATSAROS }}{ }^{2}$, J. PROTOPSALTIS ${ }^{3}$, \\ C. DROSSOS
}

${ }^{1}$ Athens General Hospital, Department of Modern Imaging Modalities, Athens, GREECE, ${ }^{2}$ IKA Oncology Hospital, Department of $\mathrm{CT}$ and MRI, Athens, GREECE, ${ }^{3}$ Tzaneio General Hospital, Department of Internal Medicine, Athens, GREECE

\section{PURPOSE}

Listeria Monocytogenes rarely involves the CNS. Purpose of this presentation is to demonstrate the role of neuroimaging in the diagnosis of intracranial infection caused by Listeria Monocytogenes.

MATERIALS AND METHODS

Two cases of Listeria Monocytogenes infection in immunocompromised hosts included CNS involvement. Brain MRI was obtained, including axial, sagittal and coronal T2-Weighted, FLAIR and T1Weighted images, before and after administration of Gd-chelate ( $0.2 \mathrm{ml} / \mathrm{kg}$ body weight). Lumbar puncture followed. CSF cultures and PCR were performed. Detailed laboratory was also done in both patients.

RESULTS

The first case showed encephalitic foci in the brainstem and the cerebellum, regions supposed to be predilective for Listeria Monocytogenes CNS involvement.

The second case demonstrated an asymmetric bilateral temporal lobe involvement, strongly suggestive for herpetic encephalitis, without obvious imaging findings of pachymeningeal or leptomeningeal involvement.

In both cases PCR in CSF was positive for Listeria Monocytogenes.

\section{CONCLUSION}

Listeria Monocytogenes does not present with a distinctive radiological pattern, therefore imaging findings should be always interpreted in the appropriate clinicolaboratory setting. Especially in immunocompromised patients with clinical and imaging suspicion of herpetic encephalitis neurolisteriosis might be also considered.

\section{P8:156}

\section{MR FINDINGS IN CHILDREN WITH MULTIPLE SCLEROSIS}

E. JURKIEWICZ ${ }^{1}$, K. KOTULSKA ${ }^{2}$, K. NOWAK ${ }^{1}$, T. KMIEC ${ }^{2}$, D. CHMIELEWSKI ${ }^{2}$, I. PAKULA-KOSCIESZA ${ }^{1}$, D. KUCZYNSKI ${ }^{2}$, K. MALCZYK ${ }^{1}$

${ }^{1}$ The Children's Memorial Health Institute, Department of Radiology, MR Unit, Warsaw, POLAND, ${ }^{2}$ The Children's Memorial Health Institute, Department of Neurology, Warsaw, POLAND

\section{PURPOSE}

Multiple sclerosis (MS) is the most common demyelinating disorder usually affects young adults. In more than $10 \%$ of cases 
MS develops before the age of 16 . Less then $5 \%$ patients are under 10 years of age.

MS in childhood differs from MS in adults in clinical and radiological features, as well as in prognosis. Therefore, MRI criteria for MS in children are still under consideration and MRI features are less well known. The aim of the present work was to present different brain and spine MR findings in children with MS, diagnosed between 2005-2008.

\section{MATERIAL AND METHODS}

MR images of 16 children (12 girls and 4 boys) aged from 7 to 16 (mean age 14.3) were reviewed retrospectively. The diagnosis of MS was made if the child fulfilled McDonald's criteria.

Brain and spinal cord MR examinations were performed on 1.5T scanner with FLAIR,T2-WI, T1-WI before and after contrast injection. Mean lesion counts were analyzed according to location, amount, size, presence of oedema and mass effect, contrast enhancement. Both Barkhof's and KIDMUS criteria were validated in our group of patients.

\section{RESULTS}

The most prevalent clinical manifestation of MS in our group of patients was optic neuritis and pyramidal dysfunction. Nine children met Barkhof's criteria for MS, and 13 met KIDMUS criteria. In all patients, MRI revealed multiple demyelinating lesions: periventricular (68\%), corpus callosum (62,5\%), deep gray matter $31.3 \%$ and infratentorial $18.7 \%$. In three children $(18,7 \%)$ large brain lesions with mass effect were present. In two cases $(12,5 \%)$ massive spinal demyelination was found. Gadolinium enhancement was present in $87,5 \%$ of cases.

CONCLUSIONS

MRI features of MS differ in children under the age of 16 and adults. KIDMUS criteria seem to be more specific for children with MS than Barkhof's criteria.

\section{P8:157}

\section{SUSCEPTIBILITY-WEIGHTED IMAGING IN PARKINSON'S DISEASE}

M. IDA, S. Sugawara, Y. Kubo, N. Yorozu, K. Hino, Y. Kawaguchi Tokyo Metropolitan Ebara Hospital, Department of Radiology, Tokyo, JAPAN

Susceptibility-weighted imaging (SWI) exploits phase shift to emphasize susceptibility changes. SWI is very sensitive in the detection of a small amount of paramagnetic iron accumulation in the brain tissue. In Parkinson's disease (PD), some histopathological studies have revealed a consistent increase of iron in the substantia nigra ( $\mathrm{SN}$ ); however, that is hard to detect by fast spinecho T2-weighted imaging (T2WI). The aim is to evaluate changes of iron accumulation in the striate and the SN using a high spatial-resolution SWI technique. 60 patients with neurologically diagnosed PD were included. All patients showed decreased MIBG uptake in the myocardium. MR examinations were performed using a clinical imager operating at 3-T with 32element head coil. SWI was acquired with a three-dimensional fast low-angle shot sequence with a TE of $20 \mathrm{~ms}$, a low bandwidth (80 Hz/pixel), a fully, first-order, velocity-compensated gradient moment nulling in all three orthogonal directions, 72 partitions, a voxel volume of $0.8 \times 0.7 \times 1.6 \mathrm{~mm}$. In 15 patients $(25 \%)$, the signal intensity of SWI was greatly reduced in the medial portion of the SN, suggesting increased iron accumulation. Although the degree of the age-related iron deposition in the basal ganglia varies from person to person, SWI demonstrated markedly increased iron deposition in the striatum, particularly in the dorsolateral part of the putamen in 12 patients $(20 \%)$. Those signal alterations were not visualized on the standard T2WI. However, there was no significant correlation between the signal reduction in the basal ganglia and the neurological severity (Yahr classification) of PD. In conclusion, SWI enables better visualization of ferric iron distribution in PD. SWI offers new insights into PD regarding iron accumulation in the basal ganglia. SWI may provide new understandings of pathophysiology and disease process in PD.

\section{P8:158}

\section{BILLATERAL SYMMETRIC SIGNAL-INTENSITY ALTERATIONS IN THE THALAMI, MORE THAN JUST SIGNAL FIXTURES}

F. MATA ESCOLANO, M. GUILLEM LLÁCER, A. GREGORIO HERNÁNDEZ, A. GONZÁLEZ-CRUZ SOLER, A. GALLARDO JUAN, J. ASENSI PÉREZ, R. ORTIZ ESTÉVEZ, V. MARTÍNEZ SANJUÁN CONSORCIO HOSPITAL GENERAL UNIVERSITARIO DE VALENCIA, VALENCIA, SPAIN

\section{PURPOSE}

To expose the entities than can cause in the thalami a billateral symmetric high signal in $\mathrm{T} 2$ sequences, which is frequently just mistaken with signal fixtures.

\section{METHODS}

We make a differential diagnosis between the different pathologies that are described to cause in the thalami a high signal-intensity in the T2 IMR images, which appears billateral and symmetric, explaining their features and including examples of them. RESULTS

There are several diseases than can be shown by symmetric bilateral signal-intensity alterations in the thalami, causing high signal-intensity in T2 sequences, making it necessary to propose a differential diagnosis between:

- Wernicke's encephalopathy.

- Prionic disease.

- Lynphoma.

- Leigh's disease.

- Billateral thalamic ischemia.

- Mytochondrial diseases (such as Kearns-Sayre syndrome, MELAS and MERRF syndromes).

- Encephalitis.

- Reye's syndrome.

- Lyme's disease.

\section{CONCLUSIONS}

We cannot always just impute to signal fixtures the bilateral and symmetric signal-intensity alterations found in T2 IMR. It's needed to make an accurate differential diagnosis, taking account of the clinical manifestations and the rest of findings made in the IRM. 


\section{P8:159}

\section{"A QUANTITATIVE EVALUATION OF DAMAGE IN NORMAL APPEARING WHITE MATTER IN PATIENTS WITH MULTIPLE SCLEROSIS USING DIFFUSION TENSOR IMAGING ON 3T MRI"}

G. GRATSIAS ${ }^{1}$, V. TSIMOURTOU ${ }^{2}$, I. TSOUGOS ${ }^{3}$, A. MARKOPOULOU ${ }^{2}$, G. HATZIGEORGIOU ${ }^{2}$, I. FEZOULIDIS ${ }^{1}$, E. KAPSALAKI ${ }^{1}$

${ }^{1}$ University Hospital of Larissa, Department of Radiology, Larissa, GREECE, ${ }^{2}$ University Hospital of Larissa, Department of Neurology, Larissa, GREECE, ${ }^{3}$ University Hospital of Larissa, Department of Medical Physics, Larissa, GREECE

\section{PURPOSE}

It is well known that White Matter (WM) in Multiple Sclerosis (MS) is damaged, even in areas that appear normal on T2, FLAIR and PD sequences. The purpose of our study is to evaluate in a quantitative manner the damage of Normal Appearing White Matter (NAWM) in patients with MS.

METHODS

In our prospective study, Diffusion weighted imaging (DWI) and Diffusion Tensor Imaging (DTI) of the brain was acquired in 44 patients with relapsing remitting MS and 27 age matched healthy adults, on a 3T (HDx, GE Medical systems, USA) MRI scanner. Fractional Anisotropy (FA) and Apparent Diffusion Coefficient (ADC) were measured. The MS population consisted of 28 women and 18 men, whereas the control group consisted of 18 women and 9 men. Follow up and identical measurements were performed every 6 months. Statistical analysis was performed by employing SPSS 11.0. RESULTS

Our results showed statistically significantly $(\mathrm{p}<0.05)$ lower FA values in the MS plaques in comparison to the values obtained from the symmetrical NAWM in our patient group. Similarly, statistically significantly $(p<0.05)$ lower FA values were found in the NAWM of the patients in comparison to the respective WM FA values in controls. We also found statistically significantly $(p<0.05)$ higher $\mathrm{ADC}$ values in the MS plaques in comparison with the symmetrical NAWM ADC values in our patient group and statistically significantly $(\mathrm{p}<0.05)$ higher ADC values in the NAWM of the patients in comparison to the respective WM ADC values in controls.

CONCLUSIONS

The present study demonstrated damage of the NAWM in MS patients, which could not be detected with conventional MRI techniques. Therefore DTI may be used in the early detection of subtle damage of WM in MS patients and also, in predicting the progression of the disease.

\section{P8:160}

\section{HYPOGLYCEMIC ENCEPHALOPATHY: DIFFUSION MR FINDINGS AND LESIONS DISTRIBUTIONS}

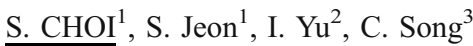

${ }^{1}$ Wonkwang University Hospital, Iksan, SOUTH KOREA, ${ }^{2}$ Elji University Hospital, Daejeon, SOUTH KOREA, ${ }^{3}$ Choongnam University Hospital, Daejeon, SOUTH KOREA

\section{BACKGROUND AND PURPOSE}

MR imaging features of hypoglycemic brain injury have not been fully established. Purpose of this study is to determine diffusionweighted imaging (DWI) findings of the hypoglycemic encephalopathy and the topographic distributions of the lesions.

MATERIAL AND METHODS

We retrospectively evaluated the DWI of hypoglycemic encephalopathy in 10 patients. The topographic distributions and signal intensities of the lesions on DWI were evaluated and apparent diffusion coefficient (ADC) values of the lesions were assessed. The ADC values of the involved lesions were compared with those of the non-involved brain parenchyma.

RESULTS

All patients had underlying diabetes mellitus (DM) with initial blood glucose level on admission ranged from $19 \mathrm{mg} / \mathrm{dl}$ to $36 \mathrm{mg} /$ dl (mean: $26.3 \mathrm{mg} / \mathrm{dl}$ ). There were bilateral symmetric diffusion restrictive lesions in cerebral cortex $(\mathrm{n}=9)$, centrum semiovale $(n=8)$, corona radiata $(n=6)$, posterior limb of internal capsule $(n=$ 6 ), and hippocampus $(\mathrm{n}=3)$ on DWI. All lesions showed hyperintensities on DWI and low ADC values. The mean ADC value of the lesions was $0.48 ; 3 / 40.126$ (mean $; 3 / 4$ SD) x 10-Cø $\mathrm{mm}(\div$ compared with non-involved brain $(0.80 ; 3 / 40.036 \times 10-(\varnothing$ $\mathrm{mm}(\mathrm{C} \div)$ and this difference was significant statistically $(\mathrm{p}=$ 0.002). Follow-up MR imagings were performed in 4 of 10 patients and showed complete remission of the lesions. CONCLUSION

DWI shows bilateral reversible hyperintensity lesions located at cerebral cortex and white matter in patients with hypoglycemic encephalopathy. The striking DWI findings may be helpful in differential diagnosis from acute stroke.

\section{P8:161}

\section{DIFFUSION TENSOR IMAGING IN HYPERTROPHIC OLIVARY DEGENERATION: CASE REPORT}

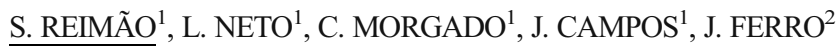

${ }^{1}$ Hospital de Santa Maria - Neurological Imaging Department, Lisbon, PORTUGAL, ${ }^{2}$ Hospital de Santa Maria - Neurology Department, Lisbon, PORTUGAL

\section{PURPOSE}

Hypertrophic olivary degeneration (HOD) is a rare neuronal degeneration usually caused by a lesion involving the triangle of Guillain and Mollaret (TGM). Although the imaging features with conventional sequences have been well described, diffusion tensor imaging (DTI) offers a possibility to map these white matter tracts. We present a case of HOD studied with DTI, focusing on the integrity of the dentate-rubro-olivary pathway.

CASE REPORT

We present the case of a 59 years-old male with a history of progressive assymetrical cerebellar ataxia for 10 years The disease was stable for 7 years after witch he developed palatal myoclonus.. Genetic tests for dominant hereditary ataxias were negative. Brain imaging was done with conventional sequences and DTI. RESULTS

Conventional brain MR showed a lesion centred in the right dentate nucleus surrounded by gliosis, causing passive dilatation of the lateral 
recess of the IV ventricle; there was an enlargement of the inferior olivary nucleus bilaterally, with increased signal on $\mathrm{T} 2$ and proton density-weighted images.

DTI study showed a marked reduction of the width of the superior cerebellar peduncle homolateral to the dentate nucleus lesion, with associated marked reduction of the anisotropy and loss of the normal blue/fiber integrity. The middle cerebellar peduncle, cortico-spinal and medial lemnsicus tract were unremarkable.

CONCLUSION

Bilateral hypertrophic olivary degeneration (HOD) is associated with a lesion affecting the triangle of Guillain and Mollaret. DTI offers a unique possibility of exploring in vivo the white matter tracts involved in these trans-synaptic changes, which in this case were thought to be due to a lesion of the right dentate and central tegmental tract.

\section{P8:162}

\section{DYKE-DAVIDOFF-MASON SYNDROME: EIGHT CASES EVALUATED BY MEANS OF MAGNETIC RESONANCE IMAGING}

T. SOLDATOS, C. SAMARA, Z. NIKOLAKOPOULOU, E. KROMMIDA, C. DROSSOS

Department of Radiology and Imaging, G. Gennimatas General State Hospital, Athens, GREECE

\section{PURPOSE}

Dyke-Davidoff-Mason Syndrome (DDMS) is a rare pre- or perinatally acquired cerebral developmental disorder that is characterized clinically by mental retardation and various neurologic signs including seizures, spastic hemiplegia and hemiparesis. The purpose of this study was to evaluate the neuroimaging findings of DDMS, particularly emphasizing in the degree of cerebral parenchymal involvement.

METHODS

We retrospectively investigated the brain magnetic resonance imaging (MRI) findings of 8 patients (6 females, age range 1443 years) with DDMS. Conventional and contrast-enhanced T1 images, as well as T2 and FLAIR images were acquired in axial, coronal and sagittal planes.

RESULTS

All patients demonstrated unilateral (62.5\% right) hemispherical hypoplasia, calvarial hypertrophy and hyperpneumatization of the paranasal sinuses, as well as ipsilateral elevation of the sphenoid wing and petrous ridge. Additional findings included unilateral deep white matter T2 hyperintensity and gliosis $(87.5 \%)$, ipsilateral mesencephalic atrophy $(75 \%)$ and asymmetry of the basal ganglia $(62.5 \%)$ as well as slimming of the corpus callosum (50\%). Varying degrees of ventricle dilatation and structural midline shift were exhibited in the MRI studies. In all cases the cerebellum was normal in terms of morphology and signal intensity.

\section{CONCLUSIONS}

In the assessment of DDMS, MRI can reveal the morhological alterations of the skull and demonstrate the extent of cerebral parenchymal involvement, thereby confirming the diagnosis.

\section{P8:163}

\section{DIFFUSION-WEIGHTED IMAGING IN NEUROCYSTICERCOSIS: SPECTRUM OF FINDINGS}

\author{
L. LUCATO $^{1}$, G. SANTOS ${ }^{1}$, L. MACHADO ${ }^{2}$, E. GASPARETTO ${ }^{1}$, \\ C. LEITE $^{1}$
}

${ }^{1}$ Clinics Hospital of the University of Sao Paulo - Department of Radiology, Sao Paulo, Brazil, Sao Paulo, BRAZIL, ${ }^{2}$ Clinics Hospital of the University of Sao Paulo - Department of Neurology, Sao Paulo, Brazil, Sao Paulo, BRAZIL

\section{PURPOSE}

To study the potential role of diffusion-weighted imaging (DWI) in neurocysticercosis (NCC) lesions, focusing on their different stages of evolution.

\section{METHODS}

Forty-three patients with diagnosis of neurocysticercosis were retrospectively analyzed. MR imaging protocol included the following sequences: T1-weighted, T2-weighted, FLAIR, T2*, gadolinium-enhanced T1-weighted, DWI and apparent diffusion coefficients (ADC) maps. All lesions presenting hyperintensity on DWI were analyzed regarding the presence of true restriction (by examining ADC maps), and their classification by stage of evolution (vesicular, colloidal, granular nodular and nodular calcified).

RESULTS

Eighteen patients presented positive DWI lesions, totalizing 35 lesions.

Hyperintensity in the scolex was appreciated in 29 lesions, including 6 in the vesicular stage and 23 in the colloidal stage. Six other lesions in the colloidal stage presented global true restricted diffusion.

\section{CONCLUSION}

In the vesicular and colloidal stage, the scolex may show hyperintensity in DWI, helping to characterize its presence, a finding with important diagnostic implications. In the colloidal phase, some cystic lesions demonstrated global restricted diffusion, possibly due to a more viscous, proteinaceous content. This information is important since NCC can be a consideration in differential diagnosis of lesions with restricted diffusion, ring enhancement and vasogenic edema, such as fungal and bacterial abscesses.

\section{P8:164}

\section{NEUROSARCOIDOSIS VERSUS MULTIPLE SCLEROSIS. OPEN-RING ENHANCEMENT SUPPORTS THE RIGHT DIAGNOSIS}

$\underline{\text { L. van den Hauwe }}{ }^{1,2}$, E. van der Holst ${ }^{1}$, J. Schelhaas ${ }^{1}$, M. Lammens ${ }^{1}$, B. Goraj ${ }^{1}$

${ }^{1}$ UMC St Radboud, Nijmegen, The Netherlands, ${ }^{2}$ Antwerp University Hospital, Antwerp, Belgium

\section{PURPOSE}

To illustrate that open-ring enhancement of white matter lesions on magnetic resonance (MR) imaging is a key feature of multiple sclerosis 
(MS) in patients presenting with both non-enhancing and enhancing brain lesions, cranial nerve enhancement and spinal cord lesions.

\section{CASE REPORT}

A 37-year-old woman was hospitalized because of rapidly progressive neurological symptoms. Clinical history and examination revealed blurred vision, left facial numbness, left-sided hearing loss, dysesthesia in both legs, as well as gait ataxia, and fatic disturbances. Previous medical history included systemic sarcoidosis with panuveitis and vasculitis.

Brain MR imaging demonstrated multiple T2 and FLAIR hyperintense lesions in the periventricular and juxtacortical white matter. Non-enhancing, homogeneous and ringlike enhancing lesions as well as open-ring enhancing lesions were observed.

Cranial nerve enhancement of the left trigeminal nerve, including the intrapontine trajectory, was noticed. More subtle enhancement of the right trigeminal nerve, left facial nerve, optic nerve/chiasma and pituitary stalk enhancement was seen. Leptomeningeal enhancement was absent.

MR angiography was normal. Spinal MR imaging displayed gadolinium-enhancing high-signal intensity T2-lesions within the cervical and thoracic spinal cord. High-resolution computed tomography (HRCT) of the chest was negative.

Relevant laboratory findings included normal angiotensin converting enzyme (ACE) levels, unique oligoclonal IgG banding in the CSF and serum.

Repeat MR imaging of the brain after 3 and 5 weeks demonstrated a combined waxing and waning pattern of the lesions.

Because of the rapid progressive clinical deterrioration of the patient, brain biopsy was performed. Pathology revealed lymphocytic inflammatory changes in the white matter and leptomeninges. Myelin staining showed white matter demyelination. Final diagnosis of MS was established.

\section{DISCUSSION}

MS and neurosarcoidosis (NS) share MR imaging features: both non-enhancing and enhancing parenchymal lesions, cranial nerve enhancement and spinal cord involvement. Open-ring enhancement after gadolinium injection has been described in the literature as being very specific for demyelinating lesions.

\section{CONCLUSION}

Open-ring enhancement of white matter lesions is in our experience a key finding allowing to differentiate between the MR pattern of NS and MS, prompting the diagnosis of the latter, which was confirmed by the pathological findings.

\section{P8:165}

\section{ANTI-NEUTROPHIL CYTOPLASMIC ANTIBODIES (ANCA)-ASSOCIATED VASCULITIS: MRI AND MRA FINDINGS}

G. ZUCCOLI $^{1}$, A. GHINOI ${ }^{2}$, N. PIPITONE ${ }^{2}$, C. SALVARANI ${ }^{2}$

${ }^{1}$ University of Pittsburgh, Pittsburgh, USA, ${ }^{2}$ Arcispedale Santa Maria Nuova, Reggio Emilia, ITALY

\section{PURPOSE}

To describe a rare case of brain involvement in anti-neutrophil cytoplasmic antibodies (ANCA)-associated vasculitis.

\section{METHODS}

Multiplanar and multiecho MRI imaging of the brain was performed emphasizing the relative T1, T2 and DWIADC signal characteristics. TOF MRA of the intracranial arteries was obtained. Clinical data were retrieved from the patient's records. RESULTS

A 61-year-old lady developed fever, widespread aches and pains, acral ischemia of the feet leading to multiple digit amputations, hemoptysis, and impaired consciousness. Laboratory tests showed raised inflammatory markers and positive cytoplasmic ANCA. 24-hour urinary protein was raised at $700 \mathrm{mg}$. Infective and autoimmune serology was otherwise negative. Chest CT showed bilateral, ground glass attenuation (GGA) consistent with pulmonary hemorrhage. Brain MRI disclosed restricted diffusion of the left hemisphere, consistent with subacute ischemic lesions, while MRA revealed multiple stenoses of the distal branches of the left middle cerebral artery.

ANCA-associated vasculitis was diagnosed and aggressive immunosuppressive therapy started with gradual clinical improvement which was confirmed by signal intensity alterations resolution.

\section{CONCLUSIONS}

Brain involvement is very rare in ANCA-associated vasculitis. MRI and MRA are very helpful in the differential diagnosis and in monitoring response to therapy.

\section{Pediatric Neuroradiology}

\section{P9:166}

\section{APLASIA OF THE INTERNAL CAROTID ARTERY: MRA FINDINGS}

\author{
$\underline{\text { K. SYRGIANNIS }}^{1}$, I. NIKAS ${ }^{1}$, M. THEOFANOPOULOU ${ }^{2}$, \\ M. GAVRA \\ ${ }^{1}$ Imaging Dpt, Agia Sofia Children's Hospital, ATHENS, GREECE, \\ ${ }^{2}$ Imaging Dpt, Ionia iatriki, Diagnostic Center, ATHENS, GREECE
}

\section{PURPOSE}

Aplasia and hypoplasia of the internal carotid artery (ICA) are rare congenital anomalies, mostly incidentally found. Symptomatic cases, usually present with acute ischemia. A number of intracranial vascular anomalies, such as aneurysms of the circle of Willis and abnormal collateral arteries are commonly associated findings. This report describes a young individual with left ICA agenesis, whose unusual presenting symptom was a mild discontinuous headache. METHODS

A 8-year old boy was admitted in our hospital complaining of intermittent headaches. Brain magnetic resonance imaging and angiography (MRI and MRA), were performed which demonstrated absence of the left ICA.

RESULTS

The left ICA was found to be missing. The left middle cerebral artery arose from the basic artery with normal flow. The right posterior communicating artery was visualized, while both anterior cerebral arteries (A2 branches) arose from the right A1. Both vertebral arteries exhibited normal flow.

CONCLUSIONS

ICA aplasia/hypoplasia is most commonly incidentally discovered as no particular symptom suggests its presence. A daily, intermittent headache of few hours duration is uncommon. The 
knowledge of this rare entity and its frequently associated conditions should prompt further evaluation. MRA is the imaging method of choice, not only in the diagnosis of ICA aplasia / hypoplasia, but also in revealing any coexisting, potentially lifethreatening conditions, i.e saccular aneurysms.

\section{P9:167}

\section{BRAIN CAVERVOUS HEMANGIOMAS IN CHILDREN : IMAGING FINDINGS}

I. NIKAS ${ }^{1}$, M. THEOFANOPOULOU ${ }^{1}$, K. SIRGIANNIS ${ }^{1}$, G. SFAKIANOS ${ }^{2}$, N. PRODROMOU ${ }^{2}$

${ }^{1}$ IMAGING DPT, AGIA SOFIA CHILDREN'S HOSPITAL, ATHENS, GREECE, ${ }^{2}$ NEUROSURGICAL DPT, AGIA SOFIA CHILDREN'S HOSPITAL, ATHENS, GREECE

\section{PURPOSE}

Cavenvous hemangiomas $(\mathrm{CH})$ are vascular malformations of the venous compartment that may affect any part of the central nervous cystem. In this study, we focus on the main diagnostic features of CNS cavernormas in pediatric patients and emphasize their differences in comparison to the adult population.

METHODS

We retrospectively studied a series of 20 children ( 11 boys, 9 girls) under the age of 16, treated for $\mathrm{CH}$ in Agia Sofia Children's Hospital, during the 10-year period from 1999-2008. Clinical and imaging features were reviewed.

\section{RESULTS}

The clinical features of $\mathrm{CH}$ are very variable. 9 patients presented typically with a sudden, intracerebal hematoma with acute focal neurologic deficits, 9 with seizures and in 2 cases the cavervoma was an incidental finding. Cavernomas were supratentorial in 15 patients $(75 \%)$ and infratentorial in the remaining $5(25 \%)$. There were 17 children with single $\mathrm{CH}(85 \%)$ and 3 children (15\%) with multiple CHs. In 2 of the later group $\mathrm{CHs}$ were a long term complication after brain irradiation for malignancy (leukemia) while the last one was a familial case. On MRI 9 patients demonstrated the typical "salt and peper" appearance, one in combination with a venous angioma, 8 had spontaneous intraparenchymal hematomas, 2 exhibited multiple foci of low T2 signal and 1 had a mixed appearance.

\section{CONCLUSIONS}

$\mathrm{CHs}$ in children differ from those in the adult population in their location and clinical presentation. It has been postulated that in children $\mathrm{CHs}$ present with more severe symptoms than in adults. The widespread use of imaging, especially MRI has significantly increased the possibility of correctly identifying $\mathrm{CHs}$, thus aiding their further management.

\section{P9:168}

\section{EPIDEMIOLOGICAL PATTERNS OF HEAD INJURY IN CHILDREN AND YOUNG ADOLESCENTS}

N. SYRMOS, C.H. ILIADIS, G. GAVRISAKIS, V. VALADAKIS, K. GRIGORIOU, D. ARVANITAKIS, F. CHARALAMPOPOULOS Neurosugical Department-CT scan Department - Venizeleio Hospital, HERAKLION, GREECE
The Venizeleio-Hospital experience-30 years retrospective study INTRODUCTION

Head injury is the most common cause of death following trauma among children in most developed and underdeveloped countries. Head injury is a disease afflicting mainly young males, and road traffic accident is the most common aetiologic factor.

MATERIAL-METHODS

This report evaluates the findings in a 30 year retrospective study done from 1978 until 2008 to establish the baseline epidemiological patterns of head injury in children and young adolescents ( $<20$ years).Data was collected using a questionnaire from the point of presentation till discharge of each head injury patient, and augmented with theatre and outpatient records, and analyzed. The Glasgow Coma and Outcome Scales were used for grading. Our Centre until early 90 was the only neurosurgical unit of the island that received referrals from private, primary and secondary facilities and other islands of Greece.

RESULTS

1. Of the 450 total patients treated, $310(68,88 \%)$ had head injuries. 2. Males were 235 (75.8\%) women were $75(24,2 \%) .3 .241(77,7 \%)$ resulted from road traffic accident, mostly from motorcycles. 4. Fractures were mostly basal 86, brain pathology was mostly cerebral contusion. 5. Treatment mostly nonoperative 197 $(63,5 \%) .6$.Mortality was $29(9,3 \%)$.

CONCLUSION

1. Head injury is a disease afflicting mainly young males, and road traffic accident is the most common etiologic factor. 2. Trauma is the main reason for neurosurgical consultation Crete and the unsafe use of roads, especially with motorcycles, remains the major cause of head injury. Glasgow Coma Scale and Injury Severity Score are important predictors of outcome in children with traumatic brain injury.

\section{P9:169}

\section{NEUROIMAGING OF PEDIATRIC ACUTE LEUKEMIA}

S. SOTO ${ }^{1}$, R. GONZALEZ ${ }^{1}$, J. QUINTANA ${ }^{1}$, H. PULGAR ${ }^{1}$, M.C. VIAL ${ }^{2}$, V. VILLALON ${ }^{1}$

${ }^{1}$ CLINICA DAVILA, SANTIAGO, CHILE, ${ }^{2}$ OXFORD UNIVERSITY, OXFORD, UNITED KINGDOM

\section{PURPOSE}

To review the different types of brain abnormalities in children with acute lymphoblastic leukemia, which are present either at diagnosis or throughout treatment.

\section{METHODS}

A retrospective analysis of brain magnetic resonance images of patients with leukemia at our institution was conducted. Studies performed during the years 2004 to 2009 with either diagnosis intention or to rule out complications were assessed. The lesions encountered were classified into two groups: those due to tumor involvement and those secondary to chemotherapy. RESULTS

The disease-related complications of leukemia observed were: chloromas, infundibular stem leukemia, hemorrhages, cranial nerve involvement and meningeal disease. Chloromas are paren- 
chyma tumoral infiltrates, visible with spectroscopy. An important pitfall in the diagnosis arises when differentiating leptomeningeal tumoral infiltration from signal changes due to intrathecal chemotherapy. As with the effects of lumbar punction, the latter is characterized by signal enhancement of sulci and basal cisterns during FLAIR. Regarding chemotherapy-related abnormalities, the following were encountered: vascular thromboses, acute Methotrexate-induced neurotoxicity, posterior reversible encephalopathy syndrome and brain atrophy.

CONCLUSION

A wide spectrum of brain abnormalities can be present at diagnosis or for the duration of treatment for leukemia. Their evolution is variable, and even though some of them are reversible others are at risk of definitive sequelae, thus the radiologist must be familiar with them in order to recognize them promptly.

\section{P9:170}

\section{MRI FINDINGS IN A PEDIATRIC PATIENT AFFECTED BY WERNICKE'S ENCEPHALOPATHY}

N. SIDDIQUI, I. Loma, G. Zuccoli

University of Pittsburgh Medical Center, Pittsburgh, PA, USA

\section{PURPOSE}

Wernicke's encephalopathy caused by thiamine (vitamin B1) deficiency can be fatal if untreated. Post-mortem studies indicate that Wernicke's encephalopathy is underdiagnosed in the pediatric population. The aim of our study is to describe pertinent MRI findings, through an unusual case of Wernicke's encephalopathy, which should raise the clinical concern for this diagnosis in the pediatric age group.

\section{METHODS}

Multiplanar and multiecho MRI imaging of the brain was performed emphasizing the relative $\mathrm{T} 1, \mathrm{~T} 2 *$ and diffusion weighted signal characteristics using a 1.5T MRI scanner. Clinical evaluation of the patient and relevant history from the family was obtained.

\section{RESULTS}

A ten year old male with a history of acute lymphoblastic leukemia presented with nonspecific mental status changes and cognitive decline. Symmetric high signal intensity alterations in the mamillary bodies, medial thalami, tectum of the midbrain, peri-aqueductal gray matter, medial vestibular nuclei, prepositus hypoglossal nuclei, substantia nigra, dentate nuclei, frontal cortex and cingulum cortex were noted on MR imaging. These findings raised the suspicion of Wernicke's encephalopathy which was confirmed on further clinical evaluation.

CONCLUSION

MR imaging is crucial in the diagnosis of Wernicke's encephalopathy, as the diagnosis in this case was not suspected prior to imaging. As demonstrated by our case report, atypical imaging features of Wernicke's encephalopathy seen in the adult nonalcoholic population can also be present in the pediatric age group; these findings include symmetric high signal intensity in the periaqueductal gray matter, mamillary bodies and medial thalamus.

\section{P9:171}

\section{SPINAL CORD EMERGENCIES: CLINICAL PRESENTATIONS AND WORK-UP OF ACUTE MYELOPATHIES IN CHILDHOOD}

\author{
F. Bauder ${ }^{1}$, A. Poretti ${ }^{1}$, I. Scheer ${ }^{2}$, A. Meyer-Heim ${ }^{3}$, A. Klein ${ }^{1}$, \\ E. Boltshauser ${ }^{1}$ \\ ${ }^{1}$ Department of Neurology - University Children's Hospital, \\ Zürich, SWITZERLAND, ${ }^{2}$ Diagnostic Imaging - University \\ Children's Hospital, Zürich, SWITZERLAND, ${ }^{3}$ Rehabilitation \\ Centre Affolter a. Albis, University Children's Hospital, Zürich, \\ SWITZERLAND
}

\section{INTRODUCTION}

Acute myelopathies are rare in childhood, but are associated with substantial risk of long-term neurological deficits. Based on five cases we discuss clinical presentation, differential diagnosis, management issues, and outcome of acute myelopathies in childhood.

CASE REPORT

All five children were previously healthy and presented with nontraumatic acute paresis and sensory loss of the lower limbs. Patient 1 is a 12 year old girl presenting with subfebrile temperature and sore throat, severe back pain, progressive paresis of the legs, sensory deficits with a sensory level, and urinary retention. Investigations showed EBV-myelitis. Patient 2 is an 11 year old boy with lower back pain radiating to the legs, followed by sudden paraparesis and bladder dysfunction. MRI showed spinal cavernoma with acute bleeding. Steroid treatment was initiated, surgical removal led to full recovery. Patient 3 is a 10 year old boy with sudden onset of paraesthesia in the thighs and a rapidly progressive paresis of the legs. He developed complete paraplegia and sensory dysfunction in the lower limbs. Spinal MRI showed T2-hyperintensity and contrast enhancement, probably due to spinal ischemia. He remained paraplegic and has neurogenic bladder and bowel dysfunction. Patient 4 is a 5 year old girl with paraparesis and pain in both legs after falling from her bike. MRI showed symmetric contrast enhancement in the anterior horns of the thoracolumbar myelon. Symptoms improved, etiology remained unclear. Patient 5 is a 13 year old girl with sudden onset of back pain, followed by paraesthesia and paraparesis, probably due to spinal ischemia, incomplete recovery followed. CONCLUSION

In acute myelopathy spinal MRI is urgent to rule out spinal cord compression and to look for inflammation or ischemia, and represents the first and most important diagnostic procedure. In most cases, history and clinical examination allow differentiation from Guillain-Barré syndrome or spinal tumor.

\section{P9:172}

\section{INTRAVENTRICULAR GLIOBLASTOMA MULTIFORME: A PEDIATRIC CASE REPORT}

\author{
A. SARSILMAZ, M. VARER, M. APAYDIN, G. COSKUN, \\ F. GELAL, O. OYAR, E. ULUC \\ ATATURK TRAINING AND RESEARCH HOSPITAL, IZMIR, \\ TURKEY
}




\section{INTRODUCTION}

Glioblastoma multiforme(GBM) is the most common lethal primary central nervous system(CNS) tumour in adults. They are rarely seen in childhood and adolescence as primary intraventricular tumours. Furthermore, few cases of solitary intraventricular GBM in adolescence were reported in the literature.

\section{CASE REPORT}

We report a case who is a seventeen-year old boy presenting with progressive disorientation, diffuse headache, vomiting and raised intracranial pressure. Computed tomography and magnetic resonance imaging(MRI) confirmed the tumour filled all of the left lateral ventricule and also invaded the surrounding parenchyma. Tumour was cystic, necrotic and multicomponental. Incomplete removal of the lesion could be achieved and pathological diagnosis was GBM. Adjuvant radiotherapy and chemotherapy was applied. The patient is still alive for one and a half year. CONCLUSION

Intraventricular tumours are relatively rare. The most common intraventricular gliomas include ependymomas. GBM is rarely seen intraventricular location and it is uncommon in childhood. The prognosis of certain intraventricular gliomas remains relatively poor. We discussed the case which is located in a very rare site and had uncommon subtype of glial tumour(GBM) for childhood. The clinical cource, radiologic findings, as well as possible treatment regimen are reviewed.

\section{P9:173}

\section{MOBIUS SYNDROME AND EPILEPSY}

G. SANTOS $^{1}$, S. PEREIRA ${ }^{1}$, T. PARREIRA ${ }^{1}$, O. BRITO ${ }^{1}$, F. SALES ${ }^{2}$, C. ROBALO ${ }^{3}$, E. MACHADO ${ }^{1}$

${ }^{1}$ Hospitais da Universidade de Coimbra - Neuroradiology Unit Department of Imagiology, Coimbra, PORTUGAL, ${ }^{2}$ Hospitais da Universidade de Coimbra - Department of Neurology, Coimbra, PORTUGAL, ${ }^{3}$ Hospital Pediátrico de Coimbra - Department of Neuropediatrics, Coimbra, PORTUGAL

\section{PURPOSE}

Mobius syndrome is a rare condition, characterized by congenital, non-progressive, facial and abducens nerve deficits, with or without craniofacial dysmorphisms and congenital limb abnormalities. Cranial nerves III, IV, VIII and XII are more rarely involved. Most cases are sporadic, but familiar occurrence has been reported. Seizures are not a common finding in patients with Mobius syndrome.

We describe a sporadic case of a female infant with facial, abducens and hypoglossal nerve paralysis, craniofacial dysmorphism and epilepsy.

\section{METHODS AND RESULTS}

A $3420 \mathrm{~g}$ infant girl was delivered through caesarean delivery at 39 weeks of gestation, with an Apgar score of 7 at one minute and 10 at five minutes. On examination, hypotonia, decreased reactivity to stimuli and craniofacial asymmetry with microretrognathia were noted, and the baby was therefore admitted to the pediatric intensive care unit.

She presented tonic seizures in the first 24 hours of life, which were medicated with phenobarbital. Facial diplegia and right abducens nerve paralysis were also noted.
The child continued to have clinical seizures, in spite of the antiepileptic medication.

There is a history of epilepsy in the family (mother and maternal grandfather). The mother was treated with carbamazepine during pregnancy, $800 \mathrm{mg} /$ day. She also took misoprostol in the first trimester of pregnancy to induce abortion.

A diagnosis of Mobius syndrome was suggested. Patient underwent MR imaging which revealed alterations in brainstem morphology, including hypoplasia of the dorsal aspect of the lower pons and of the middle cerebral peduncles. The supratentorial brain appeared normal.

\section{CONCLUSIONS}

Recurrent seizures are not usually described in patients with Mobius syndrome. In spite of that, our patient had a history of seizures soon after birth. We may assume that the same mechanism responsible for brainstem abnormality can lead to brain dysfunctions by damaging the cerebral cortex. However, no structural abnormality was present in brain hemisphere on MR imaging.

\section{P9:174}

\section{DYNAMIC SUSCEPTIBILITY CONTRAST-ENHANCED MR PERFUSION STUDIES SHOW GLOBAL CEREBRAL HYPOPERFUSION WITH FRONTAL PREDOMINANCE IN PATIENTS DEVELOPING CEREBELLAR MUTISM AFTER SURGERY FOR MIDLINE POSTERIOR}

Z. PATAY $^{1}$, N. Miller ${ }^{1}$, M. Kocak ${ }^{2}$, J. Glass $^{1}$, Q. Ji ${ }^{1}$, U. Loebel ${ }^{1}$, $\overline{\text { B. Morris }}^{3}$, A. Gajjar ${ }^{3}$, E. Reddick ${ }^{1}$

${ }^{1}$ St. Jude Children's Research Hospital, Department of Radiological Sciences, Memphis, TN, USA, ${ }^{2}$ St. Jude Children's Research Hospital, Department of Biostatistics, Memphis, TN, USA, ${ }^{3}$ St. Jude Children's Research Hospital, Department of Oncology, Memphis, TN, USA

\section{PURPOSE}

Posterior fossa syndrome (PFS) develops in about $25 \%$ of patients undergoing posterior fossa (PF) surgery and is characterized by a complex neuropsychological disturbance, which includes cerebellar mutism (CM). We hypothesized that $\mathrm{CM}$ is a form of speech apraxia, induced by surgical injury in the PF causing remote cerebral cortical dysfunction through a trans-synaptic mechanism (reversed cerebro-cerebellar diaschisis, rCCD). We designed this study using dynamic susceptibility contrast-enhanced (DSC) MR perfusion imaging to determine whether measurable changes in cerebral perfusion develop in patients with PFS and if specific cortical areas are implicated in the process.

\section{METHODS}

Eleven PF tumor patients (10M/1F, ages 3-11y) with $\mathrm{CM}$ and eleven age/gender matched patients with no $\mathrm{CM}$ following surgery were evaluated. Patients were examined 6-27 days after surgery using DSC MR perfusion imaging. Mean cerebral blood volume $(\mathrm{CBV})$ and cerebral blood flow $(\mathrm{CBF})$ was calculated for cortical gray matter divided into eight geometrically defined sectors on each slice, which were then incorporated to provide aggregate 
values of $\mathrm{CBF}$ and $\mathrm{CBV}$ for each of the eight cortical regions. Perfusion parameters of patients with PFS and controls were compared at both aggregate and slice level by region using the Wilcoxon Signed Rank Test.

\section{RESULTS}

DSC MR perfusion imaging showed a non-significant, but clear trend to global supratentorial cortical hypoperfusion and a significant decrease of $\mathrm{CBV}$ and $\mathrm{CBF}$ within frontal lobes in patients with PFS compared to controls $(\mathrm{p}<0.05)$.

CONCLUSION

Diaschisis is a sudden, complex functional impairment (affecting blood perfusion) of brain tissue in areas remote but interconnected with a site of brain injury. Global depression of cerebral cortical perfusion in patients with PFS may be explained by the abrupt cerebello-cerebral disconnection (rCCD) caused by surgical disruption of the proximal efferent cerebellar pathways. We propose that PFS represents a peculiar form of $\mathrm{rCCD}$ and $\mathrm{CM}$ is indeed an apraxia secondary to bilateral frontal lobe dysfunction.

\section{P9:175}

\section{OVERVIEW OF LIPOMATOUS LESIONS OF CNS IN PEDIATRIC PATIENTS}

M. MORTILLA, M. ANTONELLO, L. TASCIOTTI, C. FONDA Children's University Hospital Pediatric Radiology and Neuroradiology, Firenze, ITALY

\section{PURPOSE}

To review the presentation, epidemiology and classification of lipomatous lesions in the CNS in fetuses to 16 yrs old patients. To illustrate how MRI is the gold standard methods.

\section{APPROACH/METHODS}

In the last 3 years a number of pediatric patients for a total of 41 , underwent to MRI showing lipomatous lesions in the brain or in the spine. We have reviewed all these cases with the attempt to classify them.

\section{FINDINGS/DISCUSSION}

Some of the lesions detected were the reason of performing the MRI such as those that occur in spinal dysrafism but in other cases they were unespected or occasional findings.

\section{SUMMARY/CONCLUSIONS}

The major teaching points of the exhibit are: 1 . Describe the variety of the lipomatous lesions in the CNS. 2. To describe imaging characteristics on MRI. 3. Illustrate possible pitfalls.

\section{P9:176}

\section{DOES 3T MRI IN EPILEPTIC PATIENTS IMPROVE THE DIAGNOSIS?}

\section{MORTILLA, A. CICCARONE, C. FONDA}

Children's University Hospital Meyer Pediatric Radiolgy and Neuroradiology, Firenze, ITALY

\section{PURPOSE}

To illustrate the utility of the $3 \mathrm{~T}$ MRI in epileptic pediatric patients.

\section{APPROACH/ METHODS}

From June 2008 to March 2009 we have studied 42 consecutive patients affected with intractable seizures using a 3T MR scanner (Philips, Achieva).

The patients were previously studied with a $1.5 \mathrm{~T}$ scanner. Sequences included T1 IR, 3D T1, FSE T2, FLAIR, GW only, WM only, DTI and fiber tracking.

FINDINGS/DISCUSSION

19 patients out of 42 showed alterations on MRI images, 15 patients out of 19 had previously known alterations and 4 patients out of 19 showed alterations that were newly diagnosed. The previously known alterations were better depicted with the $3 \mathrm{~T}$ scanner in comparison to $1.5 \mathrm{~T}$ allowing a better surgical planning. The abnormalities detected included temporal mesial sclerosis and different types of dysplasia.

SUMMARY/CONCLUSIONS

The aim of the exhibit is to show the value of $3 \mathrm{~T}$ scanner in the diagnosis of intractable epilepsy. Since this type of scanners are more widely available, the patients affected with this disease should be promptly addressed to performed a high field MRI in order to avoid misdiagnosis.

\section{P9:177}

\section{MAPLE SYRUP URINE DISEASE- CONVENTIONAL MAGNETIC RESONANCE (MR) IMAGING WITH DIFFUSION-WEIGHTED AND APPARENT DIFFUSION COEFFICIENT (ADC) MAP}

\author{
O. KIRITSI, A. ANASTASIOU, N. VOUGIOUKLIS \\ HIPPOKRATION GENERAL HOSPITAL, RADIOLOGY \\ DEPARTMENT, THESSALONIKI, GREECE
}

\section{PURPOSE}

We present conventional magnetic resonance (MR) imaging with diffusion-weighted and ADC map in a 12-day-old infant with maple syrup urine disease.

\section{METHODS}

MR imaging was performed (after clinical and metabolic examination) on a $1.5-\mathrm{T}$ unit using standard head coil. Conventional MR with spin-echo T1-weighted images fast spin-echo T2weighted images and Diffusion- weighted images were obtained. Apparent diffusion coefficient (ADC) map was generated with dedicated software.

RESULTS

The patient was a white male neonate. His family history was negative for congenital anomalies. The neonate was full term (38 weeks); his birth weight was $3,250 \mathrm{~g}$, his height was $51 \mathrm{~cm}$ and the APGAR scores were 9 at $1 \mathrm{~min}$, and 10 at $5 \mathrm{~min}$. Four days after his birth he manifested episodes of vomiting and poor feeding. Clinical examination was performed on the 8th day of his life; truncal hypotonia and lethargy was revealed. Metabolic screenings, by Guthrie card, showed a leucine level of $15 \mathrm{mg} /$ $100 \mathrm{ml}$ (normal value less than $2 \mathrm{mg} / 100 \mathrm{ml}$ ). Plasma amino acid analysis revealed elevated leucine, isoleucine and valine levels $(1,940,1,652$ and $1,345 \mathrm{lmol} / \mathrm{l}$, respectively) suggesting the presence of Maple Syrup Urine Disease. The MRI study was 
performed on the 12th day on a 1.5-Tesla unit. Spin-echo T1weighted images did not evidence any signal abnormality, while fast spin-echo T2-weighted images showed weak diffuse hyperintensity in the cerebellar white matter, in the dorsal brainstem and thalami. Diffusion-weighted sequences showed marked hyperintensity localised in the cerebellar white matter, brainstem, cerebral peduncles, thalami, globi palidi, posterior limbs of internal capsule and corona radiate. The apparent diffusion coefficient (ADC) of these regions was markedly decreased suggesting the presence of cytotoxic edema in this Maple Syrup Urine Disease patient.

\section{CONCLUSION}

Diffusion-weighted MRI is more sensitive than conventional spinecho techniques, in detecting Maple Syrup Urine Disease brain alterations.

\section{P9:178}

\section{PROFOUND LOW INTENSITY ON DWI IN BRAINS RESISTANT TO ATROPHY}

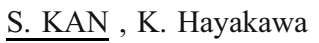

Kitasato University, Sagamihara, JAPAN

\section{PURPOSE}

To report two infant cases of severely damaged brain which showed profound low intensity at white matter on DWI.

MATERIAL AND METHODS

Case 1

A 1 year old boy had been followed up for neurofibromatosis. Prior to one day before admission, the patient had high fever. Then the patient developed generalized seizure.

After the admission, he was intubated and heavily sedated. Blood pressure was down 8 days after the admission and CPR was performed. At this time, EEG was flat and there was no respond to ABR (auditory brain-stem response). CT demonstrated diffuse brain swelling with pseudo SAH appearance. Follow up CT 2 months after the admission showed no atrophy but diffuse low density at white matter.

Case 2

A 11 month old boy was admitted because of generalized seizure . CT demonstrated low density at basal ganglia and thalamus suggesting acute necrotizing encephalopathy.

The EEG was flat and no response to ABR. Follow up CT one and half month after the admission showed no atrophy but enlarged fontanelle and diffuse low density at white matter.

\section{RESULTS}

Both cases had MRI study during chronic stage, case 15 months, case 22 months after the admission. Enlarged volume of white matter was seen and there was profound low intensity at white matter on DWI. Both cases showed enlarged epidural space. MRA was performed in case 2 and there was poor visualization of intracranial arteries.

\section{CONCLUSION}

Brain atrophy occurs usually following severe brain swelling. However when enough blood supply to brain does not maintain, normal process to atrophy might be disturbed. Profound low intensity in white matter on DWI is characteristic for this condition.

\section{P9:179}

\section{MR IMAGING IN DETECTION OF TRILATERAL RETINOBLASTOMA (TRB)-A REPORT OF THREE PATIENTS}

E. JURKIEWICZ ${ }^{1}$, I. Pakula-Kosciesza ${ }^{1}$, K. Nowak ${ }^{1}$, O. Rutynowska ${ }^{2}$ ${ }^{1}$ The Children's Memorial Health Institute, Department of Radiology, MR Unit, Warsaw, POLAND, ${ }^{2}$ The Children's Memorial Health Institute, Department of Oncology, Warsaw, POLAND

\section{PURPOSE}

Retinoblastoma (RB) is the most common pediatric intraocular neoplasm. The association of bilateral $\mathrm{RB}$ with ectopic midline intracranial tumor trilateral retinoblastoma (TRB) is uncommon.

The purpose of this study is to review brain MR examinations and tumor characteristics in patients with TRB.

MATERIAL AND METHOD

202 patients with unilateral and bilateral retinoblastoma were treated in our Institute between 1996 and 2008. The median age at the time of diagnosis of retinoblastoma was 12 months for bilateral and 22 months for unilateral tumor.

We present MR examinations of one boy and two girls with trilateral tumors, aged 16 months, 5 months, 10 months, respectively.

The MR examinations were performed with 1,5T scanner. Threeplanes T1- and T2-weighted scans before, and T1-weighted threeplanes scans after contrast administration were obtained.

RESULTS

In two cases ocular symptoms led to ophtalmological examination and diagnosis of bilateral retinoblastoma. MRI examinations revealed intracranial tumor in pineal and suprasellar region. In one case (aged 10 months) the first diagnosis was huge intracranial PNET in the midline. In further course in control MR examination intrabulbar tumor of right eye was found. Diagnosis of bilateral RB was confirmed in ophtalmological examination. All patients have no positive family history. There were no evidences of leptomeningeal spread of the tumor. All patients are alive with a follow up from 14 months to 17 months from diagnosis.

\section{CONCLUSION}

Patients with unilateral and bilateral intraocular tumors should receive brain screening by MR imaging. We recommended also that patients under the age of 3 years with the midline tumors should be carefully diagnosed for ocular neoplasms.

\section{P9:180}

\section{BRAIN MRI IN CHILDREN WITH HISTORY OF ENCEPHALITIS OF VARIED ETIOLOGY}

\author{
M. BEKIESINSKA-FIGATOWSKA ${ }^{1}$, E. JURKIEWICZ ${ }^{2}$ \\ ${ }^{1}$ Institute of Mother and Child, Department of Diagnostic \\ Imaging, Warsaw, POLAND, ${ }^{2}$ MRI Unit, Children's Memorial \\ Health Institute, Warsaw, POLAND
}

\section{BACKGROUND}

Children that recover from encephalitis suffer from neuropsychiatric sequelae of this disease, such as neurological deficits, 
behavioral changes and epilepsy. They are therefore referred to neuroimaging studies to assess the severity of post-encephalitic brain injury.

PURPOSE

To find out if there are MRI features typical of particular causative agents of encephalitis.

MATERIAL AND METHODS

We retrospectively analyzed MRI scans of 78 children after staphylococcal $(n=10)$, streptococcal $(n=6)$, inborn cytomegalovirus (CMV) $(n=44)$, herpes simplex (HSV) $(n=3)$ CNS infections, Rasmussen's encephalitis $(n=5)$ and without isolated pathogen $(n=10)$.

RESULTS

Our main findings included brain atrophy in $79 \%$ of patients, hyperintense lesions consistent with gliosis $-57 \%$, cystic lesions$43 \%$. Neither the degree nor localization of lesions were suggestive of the type of encephalitis. In cases of inborn CMV infection additionally abnormal cortical (29\%) and cerebellar (16\%) development was observed as well as calcifications. Rassmussen encephalitis appearance was typical. The gallery of images will be presented.

CONCLUSION

Encephalitis, irrespective of the causative agent, may lead to destructive changes in the brain, such as gliosis, leukomalacia and atrophy. Except for inborn CMV infection and Rassmussen encephalitis, post-encephalitic lesions are not characteristic and do not indicate the etiologic organism. Even in cases of HSVencephalitis believed to have typical frontal and temporal location, post-encephalitic malacia was seen in parietal and occipital lobes.

\section{P9:181}

\section{BRAIN MRI FINDINGS IN PRETERM BABIES VERSUS TRANSFONTANEL SONOGRAPHY-OWN EXPERIENCE. PART I}

\section{BEKIESINSKA-FIGATOWSKA ${ }^{1}$, E. HELWICH ${ }^{2}$,} H. BRAGOSZEWSKA ${ }^{1}$, A. ROMANIUK-DOROSZEWSKA ${ }^{1}$, M. RUTKOWSKA ${ }^{2}$, S. SZKUDLINSKA-PAWLAK ${ }^{1}$, E. ADAMSKA $^{2}$, M. DZWONKOWSKA ${ }^{2}$

${ }^{1}$ Institute of Mother and Child, Department of Diagnostic Imaging, Warsaw, POLAND, ${ }^{2}$ Institute of Mother and Child, Neonatology and Neonatal Intensive Care Clinic, Warsaw, POLAND

\section{BACKGROUND}

Preterm babies are at high risk for adverse neurolodevelopmental outcomes and therefore are routinely studied with transfontanel sonography (US). Bipolar opinions can still be found in the literature on the value of US and magnetic resonance imaging (MRI) in the detection of brain injury in these patients.

PURPOSE

To compare the usefulness of both imaging methods in the own material. To assess their value in the aspect of long-term prognosis.

\section{MATERIALS AND METHODS}

Material consists of 17 infants born between 24th and 31st week of pregnancy, 8 girls, 9 boys. They underwent US examinations (1-12 times), and MRI-at term equivalent. The second MRI study will be performed at 2 years corrected age. MRI was performed with a 1,5T scanner without sedation. SE/T1-, FSE/T2-, GRE/T2*-weighted images and DWI were obtained.

RESULTS

MRI changed the sonographic diagnosis in 5/17 cases $(29 \%)$ and provided more details in 4/17 (23,5\%). In 2 babies hypoplasia of the corpus callosum was diagnosed with interhemispheric cyst (in 1 of them callosal agenesis was found on US, in 1 the anomaly was not detected). In 1 infant callosal agenesis was excluded on MRI. In 2 cases posterior fossa abnormalities were observed on MRI and not visible on US. Porencephaly was not detected on US in 2 babies. Posthemorrhagic lesions were found only on MRI in 2 infants. Acute asphyxia, not deteted on US, was observed on MRI in 1 baby.

\section{CONCLUSION}

In our material MRI at term equivalent proved to be more sensitive method of detection of both hypoxic-ischemic brain injury and congenital anomalies than serial US in preterm infants. Follow-up studies will be performed in two years to assess the natural history of brain lesions and to correlate the neurological status of the children and their brain appearance.

\section{P9:182}

\section{PROTON MR SPECTROSCOPIC STUDY IN CEREBRAL} PALSY

M. BEKIESINSKA-FIGATOWSKA ${ }^{1}$, H. BRAGOSZEWSKA ${ }^{1}$, J. WALECKI ${ }^{2}$, K. SZYMANSKA ${ }^{3}$, A. JEZIOREK ${ }^{3}$, T. MAZURCZAK ${ }^{3}$, J. TRYFON ${ }^{3}$, A. ROMANIUKDOROSZEWSKA $^{1}$, E. JURKIEWICZ ${ }^{4}$, B. BIERNACKA ${ }^{5}$

${ }^{1}$ Institute of Mother and Child, Dept. of Diagnostic Imaging, Warsaw, POLAND, ${ }^{2}$ Medical Centre for Postgraduate Education, Warsaw, POLAND, ${ }^{3}$ Institute of Mother and Child, Dept. of Neurology, Warsaw, POLAND, ${ }^{4}$ Children's Memorial Health Institute, MRI Unit, Warsaw, POLAND, ${ }^{5}$ Institute of Mother and Child, Dept. of Epidemiology, Warsaw, POLAND

\section{BACKGROUND}

Cerebral palsy patients (CP) present normal brain on imaging methods in $5-25 \%$ of cases, irrespective of degree of neurological dysfunction. In these children proton magnetic resonance spectroscopy (1HMRS) becomes the method of choice for brain tissue damage assessment on neurobiochemical level. Our hypothesis is that $\mathrm{N}$-acetyloaspartate (NAA) and myoinositol (mI) levels are changed in strategic brain tissue in $\mathrm{CP}$ due to decreased number of neurons and glial reactive processes.

PURPOSE

To present the results of $1 \mathrm{HMRS}$ in children with $\mathrm{CP}$ without structural brain abnormalities, to gauge the correlation between severity of clinical dysfunction and metabolites profile in 1HMRS. MATERIAL/METHODS

Material consists of 56 children (33 m, $23 \mathrm{f}$ ), aged 2,2-15,7 years, divided into 5 subgroups: (1) right- $(n=11)$ and $(2)$ left $(n=7)$ hemiplegia, (3) diplegia $(n=19)$, (4) quadriplegia $(n=14),(5)$ other/unclasified $(\mathrm{n}=5)$. Control group consists of 62 children (34 m, 28 f), aged 1,5-18,2 years.

NAA, choline (Cho) and $\mathrm{mI}$ ratios to creatine $(\mathrm{Cr})$ were measured symmetrically in the cortex of frontal lobe, frontal operculum, temporo-occipital junction and in the basal ganglia. 
Mann-Whitney, t-Student and Wilcoxon tests were used for statistical analysis.

\section{RESULTS}

In the analyzed group metabolites ratios in the right and left hemisphere were compared. This group was also compared to the control group. The most statistically significant differences were found in basal ganglia in NAA/Cr ratios in subgroups 3 and 4. Increased $\mathrm{mI} / \mathrm{Cr}$ ratio was observed in subgroup 4 (basal ganglia). In the follow-up examinations after rehabilitation no increase in $\mathrm{NAA} / \mathrm{Cr}$ ratio has been found.

\section{CONCLUSION}

Statistically significant differences between analyzed and control group were the most pronounced in clinical subgroup 4. This most likely reflects the most severe brain injury in this group. Paradoxically these differences were found mostly in the basal ganglia and not - as expected - in the cerebral cortex. Initial results do not indicate the expected spectroscopic improvement in the rehabilitated patients.

\section{P9:183}

\section{MR IMAGING FINDINGS OF A LARGE MENINGIOMA IN A 3-YEARS -OLD CHILD: A CASE REPORT}

T. ANGILERI ${ }^{1}$, F. VERDERAME $^{1}$, T. BARTOLOTTA ${ }^{2}$, A. TAIBBI $^{2}$, M.A. BANCO $^{2}$

${ }^{1}$ VILLA SANTA TERESA, BAGHERIA, ITALY, ${ }^{2}$ POLICLINICO UNIVERSITÀ, DEGLI STUDI, PALERMO, ITALY

\section{PURPOSE}

To describe MR imaging findings of a large atypical meningioma found out in a 3-years-old infant.

\section{MATERIALS AND METHODS}

The little female patient with clinical history of epilepsy, lower limbs tremble and psychomotor deficit, underwent 1.5 MR examination before and after intravenous administration of $0.2 \mathrm{~mL} / \mathrm{kg}$ of Gadoteric acid (Dotarem ${ }^{\circledR}$ ) under sedation.

The definitive diagnosis was obtained by means of histological analysis of resected specimen. The infant was followed-up by CT and/or MR examination after surgery.

\section{RESULTS}

MR imaging showed a huge mass in the left frontal lobe, with unsure intra- or extra-axial origin, sized $10.6 \mathrm{~cm} \times 7.4 \mathrm{~cm}$. The tumour presented solid aspect in the upper part and cystic component in that lower. The mass-with solid part mainly isointense on T1-W sequences, iso-hyperintense on T2$\mathrm{w}$ sequences and presenting a strong and inhomogeneous contrast-enhancement-showed huge relationships with dura mater and determined severe mass-effect with mid line shift, subfalcine herniation of left lateral ventriculus and hypertensive dilatation of the contralateral ventriculus.

Histological examination characterized the mass as atypical meningioma WHO grade II.

No evidence of recurrence was observed at least up to the last MR follow-up performed six months after surgery.

CONCLUSIONS

Meningiomas, although common in adults, are rare in infancy and childhood. In our case surgery resection allowed a strong and progressive improvement of neurological deficits and MR imaging is useful and mandatory for either surgical planning and follow-up.

\section{P9:184}

\section{BRAIN METASTASES FROM EWING'S SARCOMA}

\author{
M. BEKIESINSKA-FIGATOWSKA ${ }^{1}$, E. JURKIEWICZ ${ }^{2}$, \\ A. RACIBORSKA ${ }^{3}$ \\ ${ }^{1}$ Institute of Mother and Child, Department of Diagnostic \\ Imaging, Warsaw, POLAND, ${ }^{2}$ Childrens Memorial Health Insti- \\ tute, MRI Unit, Warsaw, POLAND, ${ }^{3}$ Institute of Mother and \\ Child, Clinical Department of Surgical Oncology for Children and \\ Youth, Warsaw, POLAND
}

\section{INTRODUCTION}

Ewing's sarcoma accounts for approximately 30\% of primary malignant bone tumors, mainly in children and young adults. At the moment of diagnosis many patients have already metastatic spread of the disease, most often to the lungs and skeletal system. Intracerebral metastases are rare- the reported incidence is up to $4,3 \%$.

\section{PURPOSE}

To present two cases of metastatic deposits of Ewing's sarcoma within the brain tissue.

\section{MATERIALS/METHODS}

Two children: a 9-year-old boy with Ewing's sarcoma of the right iliac bone and a 16-year-old-girl with Ewing's sa of the right radius underwent brain MRI at 1,5T scanners. Standard sequences and post-gadolinium scans were obtained.

\section{RESULTS}

In case 1 three intracerebral and cerebellar masses staying in contact with the meninges were found, with edema and mass effect. In case 2 one mass in the right temporal lobe was observed. Signal intensity of the lesions and strong homogenous enhancement after gadolinium administration were similar to meningioma. CONCLUSION

Central nervous system involvement in cases of Ewing's sarcoma usually means the involvement of meninges and epidural space and results from the extension from the affected bones. Intraparenchymal metastases are much rarer but may also happen and resemble the growth and signal intensity pattern of meningioma.

\section{P9:185}

SERIAL MRI (PRE AND POST-NATAL) OF A CEREBRAL DURAL SINUS MALFORMATION WITH THROMBOSIS: A CASE REPORT

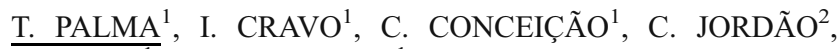
${\text { L. } \text { LOPES }^{1}, \text { C. GONÇALVES }}^{1}$

${ }^{1}$ Department of Neuroradiology, Hospital Fernando Fonseca, LISBOA, PORTUGAL, ${ }^{2}$ Ressonância Magnética Caselas, LISBOA, PORTUGAL

\section{INTRODUCTION}

Dural sinus malformation (DSM) is a rare condition that results of an incomplete maturation of the dural sinus. 


\section{CASE REPORT}

The authors report a case of a DSM with thrombosis diagnosed by magnetic resonance imaging (MRI) at 24 weeks of gestation of a male gender fetus. The patient was referred for an ectasic superior longitudinal sinus and a severe cardiomegalia on ultrasonography (US). MRI study has identified an enormous extra-cerebral torcular mass, isointense on T2 and T1 weighed images. Superior longitudinal sinus and both lateral sinuses were also extremely distended. The diagnosis of venous thrombosis was made based on the signal intensity of the dural sinuses. The cephalic biometry values on the first fetal brain MRI were low, particular for cerebellum on the 10 th centile. There was no evidence for brain parenchymal injury. Additionally there were performed more two fetal MRI's brain examinations at 28 and 35 weeks of gestation. The last fetal brain MRI showed almost complete spontaneous resolution of the venous thrombosis and sinus volume reduction. Spontaneous vaginal birth has occurred at 39 weeks. Cardiac failure has dominated the clinical condition. Cardiac colour doppler US has disclosed an inter-ventricular communication (IVC) and a persistent arterial canal. He has performed a brain MRI on the first week of life that has confirmed re-canalization of all dural sinuses, with a torcular small remnant clot. Brain parenchymal evaluation depicted cortical petechial hemorrhages. At 9 months of age the infant has a mild neurological development delay. No abnormalities were found on the evaluation of laboratory values for coagulation and thrombophilic factors.

CONCLUSION

This case report illustrates the value of the MRI on the establishing the diagnosis of the venous cerebral thrombosis and DSM in fetus. Furthermore, it provides helpful information about the natural history of fetal dural sinus thrombosis.

\section{P9:186}

\section{VOXEL-BASED MORPHOMETRY ON AN UNCONTAMINATED SAMPLE DOES NOT INDICATE GRAY MATTER REDUCTION IN ATTENTION DEFICIT HYPERACTIVITY DISORDER}

\author{
H.M. KARAKAS $^{1}{ }^{1}$, A. ONAY ${ }^{1}$, Z. BARAN ${ }^{2}$, E.T. TALI ${ }^{1}$, \\ S. KARAKAS ${ }^{2}$ \\ ${ }^{1}$ GAZI UNIVERSITY - DEPARTMENT OF RADIOLOGY - \\ SECTION OF NEURORADIOLOGY, ANKARA, TURKEY, \\ ${ }^{2}$ HACETTEPE UNIVERSITY - COGNITIVE PSYCHOPHYSI- \\ OLOGY RESEARCH UNIT, ANKARA, TURKEY
}

\section{PURPOSE}

A very recent meta-analysis showed that the morphological substrate of the historical 'minimal brain syndrome', currently attention deficit hyperactivity disorder (ADHD), is gray mater reduction. This conclusion was based on 4 out of 7 existing studies. The present study is a stringent test of the hypothesis on gray matter reduction in ADHD.

METHOD

Sample included 14 unmedicated males with ADHD-Combined type (mean age 120.21 \pm 15.27 months) and 14 age-matched (123.43 \pm 19.15 months) healthy control males. All subjects were right-handeds with IQs between 90-130. Patients were first referral cases. Exclusion criteria: Anxiety or depression, comorbid disorders, past pediatric and/or neurologic disorders, use of psychotherapeutic drugs, corrected visual/auditory defect, and electroencephalographic abnormality. Data was obtained with high-resolution 3D T1W technique. Voxel-based morphometry was performed using SPM5. A customized pediatric template set, based on NIH data, was created. Images were normalized, segmented and modulated, then smoothed with a Gaussian kernel of $12 \times 12 \times 12 \mathrm{~mm}$. Absolute gray matter volumes were calculated from original images to perform global volumetric comparisons and used as nuissance factors in ANCOVA. The difference between ADHD and control groups were analyzed using t-test for independent samples. Results were thresolded for $\mathrm{p}<0.05$ (FDR corrected) without cluster thresholding.

RESULTS

The difference between the ADHD and healthy controls with regards to gray matter volume was found to be insignificant. CONCLUSIONS

Any conclusion on the neural basis of ADHD should be based on studies that are controlled for the contaminating effect of the subject variables. In a companion study (Mangina et al., submitted to ESNR 2009), FMRI detected distributed differences between ADHD and healthy controls, possibly suggesting overemployment of neurocognitive systems. These differences were found to be in the prefrontal cortex, and parietal and visual association areas. ADHD, therefore, may affect not the morphological aspects of the brain but the way it functions in processing information.

\section{P9:187}

\section{HEMOPHAGOCYTIC LYMPHOHISTIOCYTOSIS MIMICKING GLIOMATOSIS CEREBRI}

L. CHIAPPARINI $^{1}$, G. Uziel ${ }^{1}$, M.G. Bruzzone ${ }^{1}$, N. Nardocci ${ }^{1}$, C. Vallinoto ${ }^{2}$, C. Rizzari ${ }^{2}$, A. Rovelli ${ }^{2}$, M. Savoiardo ${ }^{1}$

${ }^{1}$ Foundation IRCCS Neurological Institute C. Besta, Milano, ITALY, ${ }^{2}$ San Gerardo Hospital, Monza, ITALY

\section{PURPOSE}

To present a 13-year-old girl with symptoms of increased intracranial pressure for the last 45 days, whose brain MRI was initially interpreted as consistent with gliomatosis cerebri.

\section{METHODS}

The patient was studied with brain MRI, DWI, MRS, multiple laboratory studies including lymphocyte subset determination, multiple antibodies, and CSF examination for neurotropic viruses and cytology. RESULTS

MRI showed remarkable swelling of the cerebellum with tonsillar herniation. Hyperintensities in T2-w.i. were present in the white matter of the cerebellum and of the cerebral hemispheres. Contrast enhancement was present in the peripheral cerebellar white matter, in the peritrigonal region and in the internal capsule. ADC showed increased diffusivity. MRS showed decreased NAA and elevated Choline. A course of steroids led to a distinct reduction of the swelling, signal abnormalities and contrast enhancement. Antibodies to EBV were elevated. An episode of fever, appearance on blood tests of inflammatory signs, abnormal liver function, progressive anemia, decreased white blood cells and platelets suggested the possibility of a hemophagocytic lymphohistiocytosis (HLH) stimulated by EBV infection. The patient work-up was completed in a hemathologic department where, in addition to the 
above findings, elevated ferritin and tryglicerides, decreased NK cell activity, splenomegaly and enlarged lymph nodes were demonstrated. Bone marrow biopsy confirmed HLH. Homozygous mutation in the perforin gene was found. The patient was treated with antibiotics and immunochemotherapy. Bone marrow transplant was performed from a HLA identical brother.

\section{CONCLUSIONS}

Although the initial MRI was consistent with gliomatosis cerebri, presence of diffuse areas of enhancement are rarely seen in this condition. When they are present, they usually represent local malignant transformation. Response to steroids and development of pancytopenia were the key elements leading to the correct diagnosis. No patient with initial diagnosis of gliomatosis cerebri should be abandoned without adequate follow-up in order to exclude other pathologies.

\section{P9:188}

\section{GRISCELLI SYNDROME: A CASE REPORT AND REVIEW OF THE LITERATURE}

\section{Agarwal, K.A. Petropoulou, T. Ouyang}

Department of Radiology, Division of Neuroradiology, University of Pittsburgh Medical Center, Pittsburgh, PA, USA

\section{PURPOSE}

We present a rare case of Griscelli syndrome with involvement of the central nervous system and review the existing Englishlanguage literature. Typical and atypical neuroimaging findings on a series of CTs and MRIs will be discussed.

\section{METHODS}

A 3-and-a-half year old previously healthy boy was admitted to our institution after his third episode of fever in three month time. Extensive workup ensued including a bone marrow biopsy which revealed the presence of occasional hemophagocytic histiocytes. The presence of silvery hair was noticed and a presumptive diagnosis of Griscelli syndrome was made. During hospitalization, seizure activity was noticed which led to a series of brain MRIs included FLAIR, DWI, pre and post contrast T1W.

\section{RESULTS}

Bone marrow biopsy results showing occasional hemophagocytic histiocytes. Genetic test revealed mutations in the RAB27A gene. The first brain MRI obtained revealed numerous lesions at the gray-white matter junction in addition to brain stem involvement. A series of follow up MRI examinations revealed progressive involvement of the cerebral cortex and cerebellum ultimately involving the white matter while sparing the thalami and portions of the basal ganglia.

\section{CONCLUSIONS}

Griscelli syndrome, a rare autosomal recessive disorder is characterized by silvery hair, pigment abnormalities, severe immunologic dysfunction and central nervous system manifestations. Fulminant involvement of the brain results in a diffuse loss of cortical and deep hemispheric white matter with relative preservation of the deep gray matter, cerebellum and brain stem. While involvement of the basal ganglia has been mentioned in a prior published case report, the manifestations in the case above were far more extensive and cross at different stages with other more common pathologies.

\section{P9:189}

\section{IN UTERO COCAINE INTOXICATION: PECULIAR POST NATAL MRI FINDINGS IN AN INFANT}

\author{
G. ZUCCOLI ${ }^{1}$, F. VAGNARELLI ${ }^{2}$, N. SIDDIQUI ${ }^{1}$, S. PEDORI $^{2}$, \\ S. AMARRI ${ }^{2}$, S. PICHINI ${ }^{3}$ \\ ${ }^{1}$ University of Pittsburgh, Children's Hospital, Pittsburgh, PA, USA, \\ ${ }^{2}$ Arcispedale Santa Maria Nuova, Reggio Emilia, ITALY, ${ }^{3}$ Istituto \\ Superiore di Sanita', Roma, ITALY
}

\section{PURPOSE}

Prenatal cocaine exposure causes hypertension, placental abruption, spontaneous abortion, poor pregnancy weight gain, and under-nutrition secondary to suppression of the appetite. The offspring can also show a variety of developmental behavioral, visual, hearing, and language disorders. The aim of our study is to describe Magnetic Resonance Imaging findings in an infant prenatally exposed to cocaine. METHODS

Multiplanar and multiecho post natal MRI imaging of the infant's brain was performed emphasizing the short and long TR signal characteristics and morphological findings. Clinical evaluation of the patient and relevant history from the family was obtained. Hair testing was used to assess maternal consumption $\mathrm{f}$ drugs of abuse during pregnancy and consequent chronic fetal exposure. Maternal and neonatal hair testing was performed by a validated assay including keratin matrix digestion, extraction and analysis by gas chromatography-mass spectrometry.

RESULTS

A male newborn presented with hypoglycaemia and prolonged colestatic jaundice. At one month-follow-up, growth delay and visual impairment were detected. Sagittal T1 weighted image showed the absence of the posterior "bright spot" in its expected location within the posterior sella turcica cavity associated with pituitary stalk hypoplasia. Findings included optic nerve atrophy bilaterally. Prenatal cocaine intoxication was demonstrated by the presence of cocaine and its metabolite benzoylecgonine both in maternal hair segments corresponding to the three gestation trimesters (mean concentration: cocaine: $7.5 \mathrm{ng} / \mathrm{mg}$ hair, benzoylecgonine: $1.2 \mathrm{ng} / \mathrm{mg}$ hair) and in neonatal hair (cocaine: $5.7 \mathrm{ng} / \mathrm{mg}$ hair and benzoyeconine $1.1 \mathrm{ng} / \mathrm{mg}$ hair).

CONCLUSION

Recent reports suggest significant visual and developmental anomalies in infants prenatally exposed to cocaine. In the present study MR images correlated with clinical symptoms as demonstrated by atrophy of the optic nerves and pituitary axis anomalies. The role of MRI in depicting cocaine related Central Nervous System anomalies is discussed.

\section{Spinal Interventions}

P10:190

\section{SPINE INTERVENTIONAL PROCEDURES UNDER IMAGING- GUIDANCE: COMBINING-COMPARING FLUOROSCOPIC VIEWS WITH 3D AND CROSS-SECTIONAL CT ANATOMY}

\author{
A. CIANFONI ${ }^{1}$, D.T. Zapton ${ }^{1}$, S. Lunghi ${ }^{2}$, G. Bonaldi ${ }^{2}$ \\ ${ }^{1}$ Neuroradiology Sect.- Radiology Dept. MUSC, Charleston -SC, \\ USA, ${ }^{2}$ Neuroradiology - Ospedali Riuniti, Bergamo, ITALY
}




\section{PURPOSE/AIM}

To review pertinent normal, fluoroscopic, and CT spine anatomy for spine interventionalists.

To review the main practical principles for effective and safe imaging guidance in spine interventions.

To review fluoroscopic projections and views for percutaneous spine procedures, in combination and comparison to 3D and crosssectional CT anatomy.

METHODS

A high resolution CT whole spine model is used to show in combination and comparison the $3 \mathrm{D}$ volume rendering views, and the fluoroscopic views usually utilized to perform minimally invasive image-guided spine procedures, also obtaining the correspondent 2D multiplanar reformatted cross sectional images, for a better anatomical understanding of the important targets, and safety precautions, to be considered by the spine interventionalist. For each procedure, in the lumbar, thoracic, and cervical region, real cases images are shown.

RESULTS

The combination and comparison of the $3 \mathrm{D}$ and 2D multiplanar CT images with the fluoroscopic views utilized by the spine interventionalist further clarify the pertinent anatomy, refine the fluoroscopic access, increase confidence and safety standards.

CONCLUSIONS

Imaging guidance, with fluoroscopy or CT, allows safe performance of many percutaneous spine procedures. Knowledge of spine anatomy and imaging guidance principles is critical for spine interventionalists. This poster aim to review pertinent spine anatomy, image-guidance principles and tips, and presents fluoroscopic views of the spine, in combination and comparison with the correspondent 3D and cross-sectional CT images, for a better understanding of the relevant underlying anatomy.

\section{P10:191}

\section{INTRAFORAMINAL LUMBOSACRAL DISCAL HERNIATION:TREATMENT OF SYMPTOMATIC PATIENTS WITH INTRAFORAMINAL INJECTION OF OXYGEN-OZONE (O2-O3) MIXTURE}

T. ANGILERI ${ }^{1}$, F. VERDERAME ${ }^{1}$, T. BARTOLOTTA ${ }^{2}$, A. TAIBBI $^{2}$, M.A. BANCO ${ }^{2}$

${ }^{1}$ VILLA SANTA TERESA, BAGHERIA, ITALY, ${ }^{2}$ POLICLINICO UNIVERSITA' DEGLI STUDI, PALERMO, ITALY

\section{PURPOSE}

To evaluate the efficacy of treatment with intraforaminal injection of oxygen-ozone (O2-O3) mixture in symptomatic patients suffered from intraforaminal discal herniation.

MATERIALS AND METHODS

From January 2000 to June 2006, 92 symptomatic patients with intraforaminal discal herniation confirmed by $\mathrm{CT}$ and/or MR, underwent treatment with oxygen-ozone (O2-O3) mixture (3$4 \mathrm{~mL}$ ), corticosteroid and anaesthetic by means of intraforaminal injection.

All patients have already undergone conservative therapy for 45 days without any success with score variable from 8 to 10 at VAS test.
47/92 (51.1\%) patients presented L4-L5 disk herniation, 30/92 (32.6\%) L5-S1 disk herniation, 12/92 (13\%) L3-L4 disk herniation and, finally, three out of 92 (3.3\%) L2-L3 disk herniation.

All patients underwent two intraforaminal injections under CT guide at interval time of 15 days. VAS test was performed just before the former injection (T0), 15 days later just before the latter (T1) and one month after the second injection (T2).

RESULTS

In $59 / 92$ patients $(65 \%)$ a partial remission of the complaints was observed after the first treatment (VAS range: $4-6$ at T1). After the second treatment, one month later, in $75 / 92$ patients $(82 \%)$ a significant remission of the complaint was observed (VAS $=3$ at T2) whereas 13/92 (14.1\%) presented VAS value=7 and four out of 92 (4.3\%) did not show any improvement (VAS range: $8-10$ at T2).

Six months later the first injection, all patients underwent CT or MR examination. In 20/92 cases $(21.7 \%)$ a significant shrinkage of disk herniation was observed and all these patients have already showed good results at $\mathrm{T} 1$.

CONCLUSIONS

CT-guided intraforaminal injection of $\mathrm{O} 2-\mathrm{O} 3$ mixture associated with corticosteroid and anaesthetic is a safe and effective tool for the treatment of intraforaminal discal herniation, relieving significantly lower back pain.

\section{P10:192}

\section{DIAGNOSTIC AND ENDOVASCULAR TREATMENT OF A SPINAL EPIDURAL ARTERIOVENOUS FISTULA WITH PERIMEDULLARY DRAINAGE: CASE REPORT AND REVIEW OF THE LITERATURE}

V. DELMIRO NEVES, R. Anxionnat, A. Lebedinski, A.L. Derelle, A. Kerin Amr, S. Bracard, L. Picard

Service de neuroradiologie Diagnostique et Therapeutique - CHU Nancy, Nancy, FRANCE

\section{INTRODUCTION AND OBJECTIVES}

Epidural arteriovenous fistulas are a rare type of arteriovenous malformations of the spinal cord, usually revealed by a chronic ascending myelopathy. If not recognized in time it can lead to severe neurological sequelae. The purpose of this report is to draw attention to a curable cause of severe myelopathy, which must always be remembered.

\section{MATERIAL AND METHODS}

We report the case of a man of 57 years old, with a framework of progressive myelopathy. Initial MRI of the spine showed a T2 hyper signal of the thoracolumbar spinal cord with abnormal dilated venous network around the spinal cord, suggesting a vascular malformation. DSA demonstrated an abnormal arteriovenous at L2 level 3D angiography clearly demonstrated the arterial feeder, the location of the shunt and the perimedullar draining vein. Careful evaluation of the medullar vascularization was performed and no medullar artery was seen at the level of the fistula neither at the upper and lower levels or in the other side.

RESULTS

There was only one arterial feeder to the fistula 3D angiography helped to define an oblique working view demonstrating the course of this arterial feeder and the precise location of the shunt. Distal catheterization of this feeder was performed and allowed 
injection of a small amount of Onyx 18 which reached the shunt as demonstrated on $3 \mathrm{D}$ angiography control. Clinical issue was good. We reviewed the current medical literature.

CONCLUSION

Spinal epidural arteriovenous fistulas with perimedullary drainage are rare and may present as a progressive ascending myelopathy in a similar way than dural fistulas. In our case, 3D angiography was particularly informative to show the the precise location of the fistula. There are few reports of spinal epidural fistulas in the literature, usually treated by surgery, rarely by endovascular way and sometimes using venous road.

\section{KEYWORDS}

Spinal Epidural Arteriovenous Fistulae - Embolisation - Spinal Angiography.

\section{P10:193}

\section{KYPHOPLASTY: A TREATMENT FOR VERTEBRAL FRACTURES. FOLLOW UP AT 2 YEARS}

M. RUNGE ${ }^{1}$, J. GODARD ${ }^{2}$, J.F. BONNEVILLE ${ }^{1}$

${ }^{1}$ Radiology University hospital Jean Minjoz, Besancon, FRANCE,

${ }^{2}$ Neurosurgery University hospital Jean Minjoz, Besancon, FRANCE

\section{PURPOSE}

The goal of this exhibit is to evaluate the radiological efficacy and the clinical results of balloon kyphoplasty in 78 consecutively patients treated for a vertebral compression fracture. The second goal of this retrospective study is ti aooreciate the clinical and radiographic outcomes with 2 years follow up.

METHODS

We rewieved retrospectively 78 consecutive patients (39 male, 39 female) with 86 vertebral fractures (T5 to L5) treated with balloon kyphoplasty. All patients were included after clinical and imaging evaluation (Xray, CT scan, MRI) regardless of the underlying pathologic cause. Clinical and imaging evaluation were performed for each patient at 3, 6, 12 and 24 months after the procedure. Evaluation at each follow up time point included pain score (visual analog scale), change in pain tratment, restoration of activity, pre and post operative imaging outcome.

\section{RESULTS}

Pain score decreased significantly and immediately in all patients after the procedure with reduction $(17.1 \%)$ or stopping $(82.9 \%)$ of analgesic drugs and restoration of normal activity. These data persisted through the follow up. MRI follow up demonstrated a stabilization of vertebral body height and no complications were related. 3 patients sustained fracture in the first 2 months after the procedure at the adjacent above treated level with a new high signal on MR imaging. Pain disappeared without loss of vertebral body height with a medical treatment.

\section{CONCLUSION}

According to our results, kyphoplasty has a high success rate (pain reduction and vertebral body height restoration) and low complications rate. These results remain stable for 2 years following treatment. 